\title{
PCYT2 controls muscle health and muscle aging
}

Domagoj Cikes $^{1 *}$, Kareem Elsayad ${ }^{2}$, Erdinc Sezgin ${ }^{3,4}$, Erika Koitai ${ }^{5}$, Torma Ferenc ${ }^{5}$, Michael $^{2}$ Orthofer $^{1}$, Rebecca Yarwood ${ }^{6}$, Leonhard X. Heinz ${ }^{7}$, Vitaly Sedlyarov 7 , Nasser Darwish Miranda ${ }^{8}$, Adrian Taylor ${ }^{9}$, Sophie Grapentine ${ }^{9}$, Fathiya al- Murshedi ${ }^{10}$, Anne Abott ${ }^{11}$, Adelheid Weidinger ${ }^{11}$, Candice Kutchukian ${ }^{13}$, Colline Sanchez ${ }^{13}$, Shane J.F. Cronin ${ }^{1}$, Maria Novatchkova ${ }^{1}$, Anoop Kavirayani ${ }^{2}$, Thomas Schuetz ${ }^{1}$, Bernhard Haubner ${ }^{1}$, Lisa Haas ${ }^{14}$, Astrid Hagelkruys ${ }^{1}$, Suzanne Jackowski ${ }^{15}$, Andrey Kozlov ${ }^{11,12}$, Vincent Jacquemond ${ }^{13,16}$, Claude Knauf $^{16}$, Giulio Superti-Furga ${ }^{7,17}$, Eric Rullman ${ }^{18,19}$, Thomas Gustafsson ${ }^{18}$, John McDermot ${ }^{20}$, Martin Lowe ${ }^{6}$, Zsolt Radak ${ }^{5}$, Jeffrey S. Chamberlain 21,22, Marica Bakovic ${ }^{8}$, Siddharth Banka $^{20,23}$, Josef M. Penninger ${ }^{1,24 *}$

${ }^{1}$ IMBA, Institute of Molecular Biotechnology of the Austrian Academy of Sciences, Vienna, 1030; Austria

${ }^{2}$ VBCF, Vienna Biocenter Core Facilities, Vienna Biocenter, Vienna 1030; Austria

${ }^{3}$ MRC Weatherall Institute of Molecular Medicine, MRC Human Immunology Unit, University of Oxford, OX39DS, Oxford; UK

${ }^{4}$ Science for Life Laboratory, Department of Women's and Children's Health, Karolinska Institutet, 17165, Solna, Sweden

${ }^{5}$ University of Physical Education, Budapest; Hungary

${ }^{6}$ School of Biological Sciences, Faculty of Biology, Medicine and Health, University of Manchester, Manchester M13 9PT; UK

${ }^{7}$ CeMM Research Center for Molecular Medicine of the Austrian Academy of Sciences, Vienna; Austria

8 Institute of Science and Technology Austria (IST Austria), 3400, Klosterneuburg; Austria

${ }^{9}$ University of Guelph, Guelph; Canada

${ }^{10}$ Department of Genetics, College of Medicine, Sultan Qaboos University, Muscat;

Sultanate of Oman

${ }^{11}$ Enterosys SAS, Prologue Biotech, Labège; France

${ }^{12}$ Ludwig Boltzmann Institute for Experimental and Clinical Traumatology, AUVA Research Center, Donaueschingenstraße 13, 1200 Vienna; Austria 
${ }^{13}$ Institut NeuroMyoGène, Université Claude Bernard Lyon 1, F69622 Villeurbanne; France

14 IMP Research Institute of Molecular Pathology, Vienna, Austria

${ }^{15}$ St. Jude Children's Research Hospital, Memphis, TN 38105-3678; USA

${ }^{16}$ INSERM U1220 Institut de Recherche en Santé Digestive, CHU Purpan, Université Toulouse III Paul Sabatier Toulouse; France

17 Center for Physiology and Pharmacology, Medical University of Vienna, Vienna, Austria

${ }^{18}$ Division of Clinical Physiology, Department of Laboratory Medicine, Karolinska Institutet, and Unit of Clinical Physiology, Karolinska University Hospital, Stockholm; Sweden

${ }^{19}$ Cardiovascular Theme, Karolinska Institutet, Karolinska University Hospital Huddinge, Stockholm; Sweden

${ }^{20}$ Manchester Centre for Genomics Medicine, St Mary's Hospital, Manchester University Hospital Foundation Trust, Oxford Road, Manchester, M13 9WL; UK

${ }^{21}$ Department of Neurology, University of Washington, Seattle, WA; USA

22 Senator Paul D. Wellstone Muscular Dystrophy Specialized Research Center, University of Washington, Seattle, WA; USA

${ }^{23}$ Division of Evolution and Genomic Sciences, School of Biological Sciences, Faculty of Biology, Medicine and Health, University of Manchester, Manchester M13 9WL; UK ${ }^{24}$ Department of Medical Genetics, Life Science Institute, University of British Columbia, Vancouver; Canada

Keywords: Lipids; Genetic Disease; Ageing; Growth Failure; Progressive Muscle Weakness; Phosphatidylethanolamine; Membrane Physicochemical Properties; Membrane Stability; Exercise; Gene Therapy

\section{*Correspondence}

domagoj.cikes@imba.oeaw.ac.at (D.C.), josef.penninger@ubc.ca (J.M.P) 


\section{Abstract}

Muscle degeneration is the most prevalent cause for frailty and dependency in inherited diseases and ageing, affecting hundreds of millions of people. Elucidation of pathophysiological mechanisms, as well as effective treatments for muscle diseases represents an important goal in improving human health. Here, we show that phosphatidylethanolamine cytidyltransferase (PCYT2/ECT), the critical enzyme of the Kennedy branch of phosphatidylethanolamine (PE) synthesis pathway, has an essential role in muscle health and lifespan. Human genetic deficiency in PCYT2 causes a severe disease with failure to thrive and progressive weakness. Pcyt2 mutant zebrafish recapitulate patient phenotypes, indicating that the role of PCYT2/PE in muscle is evolutionary conserved. Muscle specific Pcyt2 knockout mice exhibited failure to thrive, impaired muscle development, progressive muscle weakness, muscle loss and accelerated ageing. Interestingly, from several organs tested, this pathology is muscle specific. Mechanistically, in muscle deficiency of PCYT2 triggers dramatic alterations of physicochemical properties of the myofiber membrane lipid bilayer, compromising membrane stability and durability under strain. We also show that PCYT2 activity declines in the aging muscles of humans and mice, and Pcyt2 gene-therapy in aged mice improved muscle strength. AAV-based delivery of PCYT2 also rescued muscle weakness in Pcyt2 knock-out mice, offering a feasible novel therapeutic avenue for rare disease patients and to alleviate muscle aging. Thus, PCYT2 plays a fundamental, specific, and conserved role in vertebrate muscle health, linking PCYT2 and PCYT2 synthesized PE lipids to severe muscle dystrophy, exercise intolerance and aging.

\section{Summary}


Collectively, skeletal muscle is the largest organ in the human body, with essential roles in mechanical support, mobility and energy expenditure. Loss and degeneration of muscle tissue, either as a result of inherited diseases ${ }^{1}$, or ageing ${ }^{2}$ severely degrades the life quality, independence, and health, of millions of people world-wide. Therefore, complete understanding of pathophysiological mechanisms that affect muscle tissue, as well as development of novel treatments, represents an important objective in modern medicine.

Eukaryotic lipidome is uniquely complex with a potential of generating 100000 lipid species ${ }^{3}$. The reason for this vast lipidome diversity is still a long-standing question in biology. Subtle and major structural differences among individual lipid species, can be seen on different levels, from subcellular compartments to cell and tissue type ${ }^{4}$. These tissue dependent differences suggest that certain organs have a functional dependency to specific lipid synthesis pathways, essential for organ health and longevity ${ }^{5}$.

In humans, a just recently discovered genetic deficiency in phosphatidylethanolamine cytidyltransferase (PCYT2/ECT), the bottle neck enzyme in synthesis of PE through the Kennedy pathway ${ }^{6}$, leads to a complex and severe disease currently diagnosed as a complex hereditary spastic paraplegia (HSP) ${ }^{7,8}$. Here, we discover a conserved, essential and specific role for PCYT2 synthesized PE in muscle health. Pcyt2 mutant zebrafish and muscle specific Pcyt2 knockout mice recapitulate several patient phenotypes, particularly failure to thrive, short stature, impaired muscle development, progressive weakness, inflammation, and accelerated ageing, resulting in shortened life span. In contrast, mice lacking Pcyt2 in other tissues are unaffected. Loss of PCYT2 in muscle triggers specific alterations in the physicochemical properties of the lipid bilayer which compromises sarcolemmal stability that is further aggravated by a mechanical strain. We show that PCYT2 activity declines in aging muscles of humans and mice, and that Pcyt2 gene-therapy in aged mice improved muscle strength. Thus, PCYT2 and PE synthesized via Pcyt2, play previously unappreciated and fundamental roles in muscle biology, linking sarcolemmal lipid bilayer disorder to muscle degeneration, strain tolerance and aging.

\section{Patients with disease-causing PCYT2 variants have failure to thrive}

PCYT2 mutations were recently discovered in human patients who manifest a complex disorder that involves developmental gross motor delay and progressive overall muscle 
weakness ${ }^{7}$. Observing these patients, we found that those with a homozygous nonsense variant NM_001184917.2:c.1129C>T (p.Arg377Ter) in PCYT2 exhibited a significantly low body weight and shorter body length starting from birth and continuing throughout childhood, puberty and early adulthood (Figure 1A,B). We also assessed patients with mutations in EPT1, which encodes the enzyme catalyzing the next and final step in PE synthesis via the Kennedy pathway (Extended Figure 1A,B). These patients have been reported to manifest similar clinical features as those with PCYT2 mutations ${ }^{8,9}$. Indeed, patients with a homozygous variant NM_033505.4:c.335G>C (p.Arg112Pro) in EPT1 also exhibited growth defects compared to unaffected children, further indicating a role for the Kennedy pathway in growth and development (Extended Data Figure 1B). Thus, mutations in two critical enzymes in PE generation via the Kennedy pathway are associated not only with previously described symptoms, such as progressive neurological deterioration, but also with stunted growth starting from birth and continuing throughout childhood.

\section{Pcyt2 deficiency in zebrafish results in stunted growth and small myofibers}

Since Ept1 loss can be partially compensated by Cept $1{ }^{10}$, we therefore focused on the bottleneck enzyme PCYT2. Given the ubiquitous tissue expression of PCYT2 ${ }^{11}$, its loss of function could potentially affect several tissues, thus contributing to complexity and severity of the disease. To gain insight into pathophysiological mechanisms, we first examined hypomorphic mutant pcyt2 zebrafish generated using the CRISPR/Cas9 system ${ }^{7}$. Similar to the human rare disease patients, pcyt2 mutant zebrafish were significantly smaller than wild type (Figure 1C).

Zebrafish and mouse models of hereditary spastic paraplegia rarely exhibit whole body growth phenotypes 12,13 14,15. In contrast, muscle development is essential for whole-body growth, and failure to thrive with short stature are well known features of muscular dystrophies ${ }^{16}$. Therefore, we examined muscle morphology in pcyt2 mutant zebrafish. We observed significantly smaller skeletal muscle fibers and reduced myofiber diameters in pcyt2 mutant zebrafish compared to controls (Figure 1D,E). These results suggest that reduced muscle size could explain the stunted growth associated with pcyt2 loss-of-function mutations in zebrafish and patients. 


\section{Pcyt2 muscle deficiency in mice impairs early muscle growth and development}

In mice, global disruption of Pcyt2 results in embryonic lethality ${ }^{17}$. Therefore, to study the role of Pcyt2 in muscle physiology and development, we generated mice with a musclespecific Pcyt2 deletion, starting earliest in the muscle development to recapitulate the human condition. Briefly, we crossed Pcyt $2^{\text {flox/flox }}$ with Myf5 promoter driven Cre mice to generate Myf5Cre-Pcyt2 offspring, given that Myf5 is the first broadly expressed myogenic regulatory factor in the developing myotome ${ }^{18}$. Pcyt2 deletion was validated by RNA sequencing (Extended Data Figure 2A,B). Lipidomic analysis of quadriceps muscle isolated from 10-day old Myf5Cre-Pcyt2 mice showed a marked and specific reduction in the levels of PE (Extended Data Figure \#A), particularly of long-chain fatty acid PE species (Extended Data Figure 3B). Myf5Cre-Pcyt2 mice were born at normal Mendelian ratios, but were significantly smaller at birth (postnatal day 1, P1) and early postnatal days (postnatal day 4, P4) gained less weight, and grew significantly less in length during the postnatal period compared to controls, as observed for both genders (Figure 1F,G; Extended Data Figure 3C-F). Neither Myf5Cre nor Pcyt $2^{\text {flox/flox }}$ littermate controls displayed a phenotype, therefore we used Pcyt $2^{\text {flox/flox }}$ littermates as controls for all subsequent experiments.

We noticed that limb muscles were smaller in Myf5Cre-Pcyt2 mice compared to controls already at P10 and still at 2 months old (Figure 1H; Extended Data Figure 3G-J). Myofiber size was significantly reduced in skeletal muscle (Figure 11; Extended Data Figure 4A). Muscle growth is mediated first by muscle satellite cell proliferation and an increase in myofibers until P7, and subsequently via myofiber hypertrophy ${ }^{19}$. The number of proliferating cells was not affected in the developing muscles of Myf5Cre-Pcyt2 mice, inferred from BrdU incorporation (Extended Data Figure 4B). Although the number and distribution of Pax7 ${ }^{+}$ muscle progenitor cells was similar in adult Myf5Cre-Pcyt2 and control mice (Extended Data Figure 4C), Myf5Cre-Pcyt2 mice showed a mild but significant reduction in myoblast fusion (Extended Data Figure 4D). To examine hypertrophic muscle growth independent of myoblast fusion, we performed synergic muscle ablation ${ }^{20}$. Muscle overloading resulted in a significant enlargement of the plantaris muscle on the un-operated limb in control mice but not in Myf5Cre-Pcyt2 mice (Extended Data Figure 4E). Together these data show that loss of Pcyt2 impairs long-chain fatty acid PE production and compromises both progenitor fusion and hypertrophic growth, leading to reduced myofiber sizes. Thus, we infer that Pcyt2 deficiency 
in muscle leads to a failure to thrive in zebrafish, mice and humans, indicating a critical evolutionary conserved role for PCYT2 in muscle biology.

\section{Muscles lacking Pcyt2 exhibit progressive weakness and inflammation}

We found that adult Myf5Cre-Pcyt2 mice exhibited apparent hindlimb clasping upon tail suspension compared to controls (Figure 2A, B), indicative of muscle weakness. Moreover, muscle strength was significantly reduced and progressively declined as Myf5Cre-Pcyt2 mice aged (Figure 2C). At 8 months of age all Myf5Cre-Pcyt2 mice developed scoliosis (Figure 2D), which was also seen in PCYT2 disease patients (Figure 2E) and in mouse models of muscular dystrophy ${ }^{21,22}$. Severe and progressive loss of muscle tissue was evident in Myf5Cre-Pcyt2 mice (Figure 2F) with a high incidence of centrally localized nuclei (Figure 2G). Furthermore, we observed tubular aggregates and inflammation in the muscles of 12-15 months old Myf5Cre-Pcyt2 mice (Figure 2H, Extended Data Figure 5A-C). Consequently to the overall muscle weakness, the Myf5Cre-Pcyt2 mice developed secondary ageing like osteopenia contributing to overall frailty (Figure 2I). As a result, Myf5Cre-Pcyt2 mice had a significantly decreased survival rate compared to controls (Figure 2J).

As expected, myopathic changes and consequent progressive sarcopenia had a dramatic effect on whole-body metabolism. At 8 months of age, blood glucose levels were significantly lower in Myf5Cre-Pcyt2 mice; food consumption was also markedly decreased (Extended Data Figure 5D-E). Although Myf5Cre-Pcyt2 mice were less active (Extended Data Figure 5F), total energy expenditure relative to body weight was significantly increased in both light and dark periods (Extended Data Figure 4G,H), indicating that the muscle wasting phenotype is indeed associated with markedly altered metabolism.

\section{Pcyt2 is specifically required in muscle}

Myf5Cre is active in precursors of both skeletal muscle and brown adipose tissue (BAT) (but not heart muscle) ${ }^{23}$. PE species were reduced in the BAT of Myf5Cre-Pcyt2 mice, but to a markedly lower extent than in the skeletal muscle (Extended Data Figure 6A,B). Importantly, loss of Pcyt2 did not affect in vitro differentiation of adipocyte progenitors into brown fat, BAT activity, or levels of the brown fat marker UCP1 in BAT (Extended Data Figure 6C-F). In addition, mitochondrial ultra-structures, content and respiration were not significantly 
affected in BAT of Myf5Cre-Pcyt2 mice (Extended Data Figure 6G-J). Moreover, we crossed Pcyt2 $2^{\text {flox/flox }}$ mice to the AdipoQCre line to remove Pcyt2 specifically in white and brown adipose tissue ${ }^{24}$ and did not observe any differences in growth or blood glucose, nor any apparent pathologies (Extended Data Figure 7A-C). Tissue-specific deletion of Pcyt2 in motor neurons (Mnx1Cre; Extended Data Figure 7D-F), gut epithelium (Villin1Cre; Extended Data Figure 7G-I), and epithelial cells within the mammary gland and skin (K14Cre, Extended Data Figure $7 \mathrm{~J}-\mathrm{L}$ ), neither resulted in apparent developmental deficiencies nor any degenerative phenotypes up to 12 months of age. This intriguing lack of severe phenotypes upon loss of Pcyt2-derived PE suggests that this lipid does not play an essential role in the plasma membranes or mitochondrial membranes of these tissues ${ }^{25-31}$.

To further explore muscle-specific deletion of Pcyt2, we crossed Pcyt $2^{\text {flox/flox }}$ mice with MckCre animals to generate MckCre-Pcyt2 offspring. In contrast with Myf5, McK is not active in the early myotome formation and is expressed later in mature muscle ${ }^{32,33}$. MckCre-Pcyt2 mice displayed muscle weakness phenotype with a late onset at 18 months of age (Extended Data Figure 7M,N), consistent with previous observations that MckCre-Pcyt2 mice up to 4 months of age did not display degenerative phenotypes ${ }^{34}$. Critical membrane myotome development is established early in utero, facilitated by addition and fusion of muscle satellite cells (MSC) and adult myoblasts and perturbations in these early events manifest as early occurring and severe dystrophies ${ }^{35,36}$. Moreover, membrane PE lipid has a slow turnover with very long half-life ${ }^{37}$. Given that McKCre is active very late in muscle formation (peak Cre activity P10), and only in mature muscle without affecting the early myotome, MSC and myoblasts ${ }^{32,33} 35,36$, this coupled with slow membrane lipid turnover delays the disease effects of impaired de-novo membrane organization as observed in PCYT2 patients. Beneficial effects observed in young MckCre-Pcyt2 mice ${ }^{34}$ likely represents an early compensatory mechanism. Overall, these data show that genetic inactivation of Pcyt2 and Pcyt2 synthesized PE specifically in muscle, results in progressive muscle weakness, muscle wasting, atrophy and a shortened lifespan.

\section{Loss of Pcyt2 directly alters sarcolemmal lipid bilayer organization and rigidity}


At the cellular level, $\mathrm{Ca}^{2+}$ release and $\mathrm{Ca}^{2+}$ uptake in isolated myofibers were unaffected in muscles from 6-month-old Myf5Cre-Pcyt2 mice manifesting gross phenotypes (Extended Data Figure $8 \mathrm{~A}, \mathrm{~B})$, suggesting that sarcoplasmic reticulum function was preserved. PE conjugation to ATG8 is necessary for autophagy ${ }^{38}$. However, the levels of PE-conjugated ATG8 were also unaltered in muscles of 6-month-old Myf5Cre-Pcyt2 mice (Extended Data Figure 8C), suggesting that LC3 conjugation to PE phospholipids was not dysregulated. Moreover, complete genetic inactivation of autophagy and LC3 PE lipidation in Myf5-derived lineages induces brown fat over-activation, but does not lead to muscle weakness, degeneration and muscle dystrophy ${ }^{39}$; both these phenotypes contrast sharply with our Myf5Cre-Pcyt2 mice. We infer that the PE forms generated by Pcyt2 are not critical for ATG8-PE conjugation. Overall, these data suggest that loss of Pcyt2 does not affect PE-modifications in autophagy or sarcoplasmic reticulum.

Primary deficiencies in mitochondrial respiration display either reduced mitochondrial mass, or increased mitochondrial mass due to compensatory mitochondrial biogenesis ${ }^{40-46}$ Deletion of mitochondrial respiratory regulators in muscle via MckCre results in early onset and severe muscle wasting ${ }^{47-50}$. Postnatal muscle-specific deletion of the mitochondrial route of PE synthesis via PSD, results in a severe muscle phenotype with rapid manifestation ${ }^{51}$. In contrast, in Myf5Cre-Pcyt2 mice, the ultrastructural morphology and contents of mitochondria appeared unchanged in muscles although the pathology was apparent (Extended Data Figure 8D,E). We hypothesize that muscle mitochondrial membranes differ from the sarcolemma with respect to PE synthesis, which could reflect their different evolutionary origins. We observed impaired mitochondrial respiration in older mice (Extended Data Figure 8F,G) increased levels of cellular reactive oxygen species (ROS), as determined by anti-oxidant catalase activity and protein oxidative damage (Extended Data Figure $8 \mathrm{H}, \mathrm{I})$, for which we believe are secondary consequences of myofiber stress. Indeed, the muscles of Myf5Cre-Pcyt2 mice showed upregulation of the cellular stress pathway components (pJNK, Foxo1) and of markers for muscle wasting (Atrogin, MuRF1, Fbx031) (Extended Data Figure 8J,K).

Our muscle lipidomics data showed a significant decrease in PEs containing long chain fatty acids (FAs)(Extended Data Figure 2A,B), which are abundant membrane lipids ${ }^{52}$. Impaired sarcolemmal stability due to indirect perturbations causes myofiber degeneration in muscular 
dystrophies ${ }^{53}$. Therefore, we hypothesized that the reduced abundance of PEs containing long chain FAs in Myf5Cre-Pcyt2 mice might directly affect formation and stability of the sarcolemmal lipid bilayer, driving the muscular pathology. To test this hypothesis, we first evaluated whether the organization of the sarcolemmal lipid bilayer was altered in Myf5CrePcyt2 mice. Spectral imaging of NR12S-stained giant plasma membrane vesicles (GPMVs) provides structural information of lipid bilayers in their native compositional complexity, which enables measurements of membrane packing, affecting bending rigidity ${ }^{54}$. We derived the parameter of general polarization (GP), with higher values corresponding to tightly packed, rigid lipid bilayer and lower values corresponding to loosely packed, and soft bilayer. Strikingly, polarization microscopy of GPMVs derived from Myf5Cre-Pcyt2 myoblasts already displayed loosely packed and less rigid lipid bilayer as compared to control myoblasts derived GMPVs (Figure 3A-B). To further address if the membrane bilayer changes persist in terminally differentiated myofibers, we assessed GPMVs from myofibers immediately after isolation from the tissue (Figure 3C). GPMVs isolated from myofibers of Myf5Cre-Pcyt2 mice again showed significant reduction of lipid packing and soft lipid bilayer. (Figure 3D). We infer that lipid bilayer membrane organization and rigidity is disrupted in both myoblasts and terminally differentiated myofibers that lack Pcyt2.

To directly address how these structural-chemical changes of the membrane lipid bilayer affect mechanical properties of the whole myofibers, we employed high-resolution Brillouin Light Scattering Microscopy (BLSM) on isolated myofibers; BLSM is used to assess the elastic modulus of bio-materials ${ }^{55}$. Scans using BLSM revealed a significant reduction in the surface stiffness of myofibers isolated from Myf5Cre-Pcyt2 mice compared to controls (Figure 3E-F). Atomic force microscopy on single myofibers further confirmed that Myf5Cre-Pcyt2 myofibers have a higher degree of membrane deformity after applying pressure at a nanoscale level and reduced membrane stiffness compared to Pcyt2-expressing muscle cells (Figure 3G,H). Thus, loss of Pcyt2 in skeletal muscle fibers results in an altered architecture of membrane lipid bilayers, directly perturbing sarcolemmal lipid bilayer mechanical properties of rigidity and stiffness.

\section{Pcyt2 deficiency leads to impaired sarcolemmal integrity and impaired strain} tolerance 
An intact cell membrane architecture is critical for membrane barrier function. In particular, the sarcolemma undergoes recurrent injury, for example via mechanical strain such as during exercise, and needs to be repaired for proficient skeletal muscle function ${ }^{56}$. Firstly, to determine if the perturbed architecture of the sarcolemma in Myf5Cre-Pcyt2 mice leads to altered permeability, we injected 6-month-old control and Myf5Cre-Pcyt2 mice intraperitoneally with Evans blue $(<1 \mathrm{kDA}){ }^{57}$. We observed an extensive accumulation of Evans blue in the quadricep muscles of Myf5Cre-Pcyt2 mice relative to controls (Figure 4A). To further explore sarcolemmal stability, we induced laser mediated membrane microinjury on freshly isolated myofibers and quantified the extent of damage in real time, by measuring intracellular entry of the fm1-43 dye, a styryl dye that is nonfluorescent in aqueous solution (extracellular) but increases its fluorescence after intracellular entry and binding to lipid vesicles ${ }^{58}$. Control myofibers displayed minimal influx of fm1-43 following laser injury, whereas Myf5Cre-Pcyt2 myofibers showed high fragility as seen from increased permeability to fm1-43 in response to the defined laser injury (Figure 4B; Video S1-S4). Thus, a lack of Pcyt2 results in compromised myofiber membrane permeability and susceptibility to damage in vitro.

To directly address sarcolemmal durability to strain in vivo, we subjected littermate control and Myf5Cre-Pcyt2 mice to a downhill running regimen on a treadmill; such eccentric exercise is a potent inducer of sarcolemma strain, while having very low energy requirement compared to concentric exercise of the same load ${ }^{59}$. During the early acclimatization phase (low speed downhill running; 4 meters $\mathrm{min}^{-1}$ for $40 \mathrm{~min}$ ) and the intermediate phase (4 meters $\mathrm{min}^{-1}$ for 40 min plus 9 meters $\mathrm{min}^{-1}$ for 20 minutes) of the eccentric exercise, Myf5Cre-Pcyt2 mice performed similarly to their littermates (Figure 4C). However, during the late stress phase (20 meters $\mathrm{min}^{-1}$ for 20 minutes), Myf5Cre-Pcyt2 mice failed to complete the exercise (Figure 4C; Video S5). We next analyzed the skeletal muscle before and after the last phase of training. The weights of the quadricep and gastrocnemius muscles increased after training in control mice In contrast, the muscles of Myf5Cre-Pcyt2 mice failed to undergo physiologic hypertrophy (Extended Data Figure 9A).; instead their quadriceps muscle exhibited foci of inflammatory cell infiltrates, ectopic fatty cell deposits and fibrosis, resulting in a marked overall myositis score (Figure 4E-F, Extended Data Figure 9B). We performed immunostaining for Dysferlin, a protein critical to sarcolemmal membrane repair, and found that it was 
aberrantly localized in Myf5Cre-Pcyt2 quadricep muscles after training (Extended Data Figure 9C). In addition, we observed disorganized F-actin networks in Myf5Cre-Pcyt2 mice after training (Extended Data Figure 9D). Of note, the number of $\mathrm{Pax} 7^{+}$progenitors in the quadricep muscle appeared similar between age-matched trained control and Myf5Cre-Pcyt2 mice (Extended Data Figure 9E). Thus, Pcyt2 PE synthesis is required for sarcolemmal durability under strain, preventing excessive muscle damage and enabling physiologic hypertrophy during eccentric exercise.

\section{Muscle-specific Pcyt2 gene therapy rescues muscle weakness in Pcyt2 mutant mice}

Currently, there is no treatment for the disease caused by PCYT2 deficiency. Since therapies have made great advancements and shown promise in the treatment of rare diseases ${ }^{60,61}$ we sought to ameliorate the muscle weakness Myf5Cre-Pcyt2 mice using muscle specific delivery of HA-tagged Pcyt2. Briefly, we cloned mouse Pcyt2 under the control of the muscle-restricted creatine kinase 8 (CK8) promoter/enhancer into an AAV-based gene delivery vector, in this case AAV6 ${ }^{62,63}$. Of note, AAV6 based gene therapies are currently in clinical trials for Duchenne muscular dystrophy ${ }^{63}$. AAV6:CK8:Pcyt2-HA vector or saline (as control) was injected into 4 day old Myf5Cre-Pcyt2 mice and grip strength was tested in adult mice (Figure 4G). Strikingly, in the AAV6:CK8:Pcyt2-HA treated mice we observed a significant increase in grip strength (Figure 4H, Extended Data Figure 10A). Thus, muscle specific AAV based gene delivery of Pcyt2 can efficiently ameliorate muscle weakness as a result of genetic Pcyt2 deficiency.

\section{Muscle-specific Pcyt2 gene therapy improves muscle strength in aging}

Sarcopenia and progressive muscle atrophy are critical determinants of frailty in aging. Muscle aging is commonly associated with diminished membrane integrity, increased susceptibility to damage, and diminished repair after exercise ${ }^{64-71}$. As Myf5Cre-Pcyt2 mice displayed degenerative features that are also found in aging muscles, we assessed a potential 
role of Pcyt2 in muscle aging. Indeed, Pcyt2 mRNA expression and enzymatic activity in quadricep muscle were reduced in aged, pre-sarcopenic mice compared to young mice (Figure 4I, Extended Data 10B). Importantly, PCYT2 activity and levels were substantially decreased in quadricep muscle biopsies of otherwise healthy 45-62 year-old compared to 2030 year-old humans (Figure 4J, Extended Data 10C,D). Thus, in mice and humans, Pcyt2 expression and even more activity decline with aging.

To test whether increasing the levels of Pcyt2 can improve muscle function in aged mice, we aimed to rejuvenate aged muscles via overexpression of Pcyt2. AAV6:CK8:Pcyt2-HA or saline (as control) were injected retro-orbitally into 24 month-old male C57B6/J mice and the expression of Pcyt2-HA in the quadricep muscle was confirmed after 2 months (Extended Data 10E). Remarkably, we observed significantly improved grip strength at 1 and 2 months after gene delivery in AAV6:CK8:Pcyt2-HA mice compared to control mice (Figure 4K, Extended Data Figure 10F). Thus, Pcyt2 overexpression has a beneficial effect on muscle strength in aged mice.

In summary, our results uncover a critical and conserved role for Pcyt2 and Pcyt2-regulated lipid biosynthesis in the biophysical properties of muscle membranes. We show that loss of Pcyt2-dependent lipid biosynthesis causes a previously unrealized form of muscular dystrophy, characterized by aberrant muscle development, progressive muscle weakness and wasting, failure to thrive and shortened lifespan. Our work reveals that with Pcyt2 deficiency reduction of long chain PE synthesis, changes the sarcolemma lipid bilayer, yielding a loosely packed and soft lipid bilayer. This disruption of lipid bilayer physicochemical properties specifically compromises muscle membrane stability, affecting muscle growth, resistance to mechanical damage due to contraction, further causing muscular decline, dystrophy and shortening of lifespan. This form of muscular dystrophy is unique, in that a lipid species provides mechanical support to the bilayer, as opposed to other forms of dystrophies that are caused by aberrations of cytoskeletal or extracellular proteins ${ }^{22}$, and may thus also have distinct therapeutic implications. On a cellular level, disruption of bilayer homeostasis triggers myofiber stress, increases ROS levels which easily penetrates less viscous bilayers ${ }^{72,73}$ and activates the JNK-FoxO1 axis of muscle atrophy. 
Membrane organization occurs very early in skeletal muscle development. Mouse models that initiate gene loss very early in myotome development, faithfully recapitulate muscular dystrophies $22,35,36$. Whereas muscular dystrophy is typically caused by the disruption of proteins that support the sarcolemma, we show that loss of Pcyt2 leads to intrinsic changes of the membrane lipid bilayer, thus representing a unique disease mechanism.

Our findings indicate that the muscle tissue is especially vulnerable to loss of Pcyt2 and Pcyt2 synthesized PE. It is well established that distinct tissues have a diverse membrane lipid composition ${ }^{4}$ and may be differentially dependent on Pcyt2. Indeed, mining the Achilles Depmap data portal, which contains gene essentiality scores from 769 cell lines ${ }^{74}$, we found that Pcyt2 is not essential for a large majority of the tested cell lines ( $4.8 \%$ dependent cell lines). For comparison, many more cell lines (54\% dependent cell lines) are dependent on choline-phosphate cytidylyltransferase (Pcyt1a), a bottleneck enzyme for synthesis of phosphatidylcholines in the parallel branch of the Kennedy pathway. The muscle dependency on Pcyt2 derived PE might be explained by the general chemical properties of PE lipids. Increasing PE concentrations increase the viscosity of the liposomes ${ }^{75}$, therefore we hypothesize that the constant mechanical strain and contraction of the myofibers render muscle membranes dependent on PE for mechanical support. The essential dependency of myofibers on Pcyt2 derived PE compared to other cell types, is supported by our findings from various tissue-specific mutants.

Muscle loss and sarcopenia are critical hallmarks of aging, and a leading cause of frailty and dependency. We found that Pcyt2 levels and activity markedly declined in muscles from aged rodents and humans. Of note, we chose our time-point for this analysis at a stage that is presarcopenic to avoid secondary effects of frailty. Decreased expression of Pcyt2 mRNA was recently observed in aged rat muscles ${ }^{76}$. It is possible that this reduction occurs as a consequence of a metabolic switch in aged muscle, which in aging appears to be more directed towards triglyceride and cholesterol synthesis ${ }^{77}$. Indeed, low density lipoprotein, cholesterol oxysterols or LXR (liver X receptor, a transcriptional regulator of cholesterol, fatty acid, and glucose homeostasis) regulate and inhibit Pcyt2 ${ }^{78}$. Importantly, our data show that Pcyt2 and long chain PE membrane lipids are critically involved in muscle health. Muscle specific AAV based Pcyt2 gene therapy ameliorated the muscle weakness in mutant mice, thus paving the way for treatment of the severe human disease developing as a result in 
bioRxiv preprint doi: https://doi.org/10.1101/2022.03.02.482658; this version posted March 3, 2022. The copyright holder for this preprint (which was not certified by peer review) is the author/funder. All rights reserved. No reuse allowed without permission.

PCYT2 mutation. Strikingly, we also found that increasing Pcyt2 expression in aged mice improves muscle strength. Given the decline of Pcyt2 in aging and the beneficial effect upon Pcyt2 gene delivery into aged mice, Pcyt2 upregulation could be considered as a potential treatment to improve muscle frailty, a critical issue in our aging world. 


\section{Acknowledgements}

We would like to thank all members of our laboratories for helpful discussions and Life Science Editors for editorial support. We are grateful to Vienna Biocenter Core Facilities: Mouse Phenotyping unit, Histopathology unit, Bioinformatics unit, Biooptics unit, Electron microscopy unit and Comparative medicine unit. We are grateful to the Lipidomics facility and K. Klavins at the CeMM Research Center for Molecular Medicine of the Austrian Academy of Sciences for assistance with lipidomics analysis. We thank A. Klymchenko (Laboratoire de Bioimagerie et Pathologies Université de Strasbourg, Strasbourg, France) for providing the NR12S probe. We are thankful to the Sen. Paul D. Wellstone Muscular Dystrophy Cooperative Specialized Research Center Viral Vector Core facility (Seattle, US) for AAV6 production. We would also like to thank Kevin P Campbell and Mary E Anderson (University of lowa, Carver College of Medicine, lowa US) for advice on muscle tissue handling. J.M.P. is supported by IMBA, an ERC Advanced Grant, a Wittgenstein award, the T. von Zastrow foundation, and a Canada 150 Research Chair in functional genetics. R.Y. is supported by a PhD studentship from the Wellcome Trust $(203995 / Z / 16 / Z)$. J.S.C. is supported by grants RO1AR44533 \& P50AR065139 from the US National Institutes of Health. C.K. is supported by a grant from the Agence Nationale de la Recherche (ANR) (ANR-18-CE14-0007-01). .AV.K. is supported by European Union'sHorizon 2020 research and innovation programme under theMarie Skłodowska-Curie grant agreement No 67544, an Austrian Science Fund (FWF) No P-33799, AW is supported by Austrian Research Promotion Agency (FFG) project No 867674. E.S. is supported by a SciLifeLab fellowship and Karolinska Institutet Foundation Grants. Work in the GSF laboratory is supported by the Austrian Academy of Sciences, the European Research Council (ERC AdG 695214 GameofGates) and the Innovative Medicines Initiative 2 Joint Undertaking (grant agreement No 777372, ReSOLUTE). 


\section{Author Contributions}

D.C. together with J.M.P. designed and supervised the mouse study, and wrote the manuscript with the input from the co-authors. All experiments were performed and established by D.C. with the following exceptions: K.E. performed Brillouin light scattering microscopy measurements. E.S. performed GPMVs isolation and image analysis with assistance from D.C. E.K. performed synergic ablation assay and analysis under supervision form Z.R. T.F. performed treadmill experiment with assistance from D.C. and under supervision from Z.R. R.Y. collected and analyzed zebrafish models under supervision from M.L. L.X.H. and V.S. performed lipidomics analysis under supervision of G.S.F. A.T. and S.G. performed Pcyt2 enzyme activity analysis under supervision of M.B. A.A. performed in vivo brown fat activity and Ucp1 RT-PCR analysis under supervision from C.K. A.W. performed respiration analysis under supervision from A.K. C.K. and C.S. performed myofiber calcium kinetics experiment, with analysis and supervision under J.V. S.J.F. assisted with western blot experiments. M.N. performed bioinformatic analysis of efficiency of Pcyt2 deletion in mice. A.K. performed myositis scoring. N.D.M. performed atomic force microscopy measurements and data analysis with assistance from D.C. T.S. and B.H. assisted in histological analysis. L.H. performed AAV6 i.v. injections. A.H. assisted in tissue sampling for western blot experiments. S.J. provided Pcyt2 floxed mice. E.R. and T.G. collected human muscle biopsies. J.S.C. generated AAV6 vector and provided guidance with all AAV experiments. J.M. and S.B. identified PCYT2 human mutant carriers, collected growth data and generated growth curves.

\section{Declaration of Interests}

D.C. and J.M.P. have applied for a patent via IMBA/UBC to use Pcyt2 to restore muscle health. C.K. is one of the co-founders of Enterosys S.A. (Labège, France). 


\section{Figure legends}

\section{Figure 1. Phenotypes of human PCYT2 rare disease mutation and pcyt2 mutant zebrafish.}

(A) Body weight and (B) height gain of patient carrying the homozygous nonsense variant 3c.1129C>T (p.Arg377Ter) in the PCYT2 gene. Controls indicate WHO standards of median weights and heights at the respective ages +/- 2 standard deviations (SD). (C) Representative appearance and quantifications of body length of control and hypomorphic pcyt2 mutant zebrafish at 14 months post fertilization. $n=4$ for each group. (D) Representative muscle sections and muscle myofiber sizes of control and hypomorphic pcyt2 zebrafish. Scale bar $50 \mu \mathrm{m}$. (E) Distribution of muscle myofiber diameters of control and hypomorphic pcyt2 zebrafish. For $(D)$ and $(E) \geq 70$ myofibers of the same anatomical region were analyzed, $n=4$ animals per group. Data are presented as means \pm SEM. ${ }^{*} p<0.05$ (unpaired Student t-test). (F) Body weight gains of control and Myf5Cre-Pcyt2 littermates on standard chow diet. $\mathrm{n}=11$ 15 per group. (G) Appearance of 4 day old (P4) and 56 day old (P56) control and Myf5CrePcyt2 littermates. Scale bars are $1 \mathrm{~cm}$ for P4 and $2 \mathrm{~cm}$ for P56. (H) Skeletal muscle appearance (quadriceps) isolated from 10 day control and Myf5Cre-Pcyt2 littermate mice. (I) Representative cross sections and distribution of skeletal muscle myofiber diameter sizes from 6 month old control and Myf5Cre-Pcyt2 mice. Myofibres were imaged using 10X magnification with $\geq 400$ myofibers analyzed per mouse. $n=4$ animals per group. Scale bar $100 \mu \mathrm{m}$.

Figure 2. Inactivation of Pcyt2 in mice leads to progressive weakness, muscle atrophy, inflammation and accelerated ageing.

(A) Representative images of 6 month old control and Myf5Cre-Pcyt2 mice and (B) quantification of progressive worsening of hind limb clasping (B). Each dot represents one mouse, values are average of three measurements per mouse; scale bar $1 \mathrm{~cm}$. (C) Agedependent decline in grip strength in control and Myf5Cre-Pcyt2 littermates. Each dot 
represents one mouse, values are average of three measurements per mouse. (D) Typical scoliosis appearance and scoliosis severity in 8 month old control and Myf5Cre-Pcyt2 mice. $\mathrm{n}=4-7$ per group. (E) Evident scoliosis (arrows) in a patient carrying the homozygous nonsense variant NM_001184917.2:3c.1129C>T (p.Arg377Ter) in PCYT2. (F) Representative image and quantification of relative muscle mass changes of 12 month old versus 6 month old control and Myf5Cre-Pcyt2 littermates. QA, quadriceps; GC, gastrocnemius; TA, tibialis anterior muscles. Scale bar = $1 \mathrm{~cm} ; \mathrm{n}=7$ per group. (G) Quantification of myofibers with central nuclei in quadriceps muscles from 8 month old control and Myf5Cre-Pcyt2 mice. $n=3$ mice per group. Scale bar $100 \mu m$. (H) Muscle inflammation as determined by H\&E staining. Data are from 12 month old mice. Data are representative for $n=4$ mice per group. Scale bar $100 \mu m$. (I) Representative cross section of tibial bone in 12 month old control and Myf5Cre-Pcyt2 mice with quantification of tibial bone cortical thickness. Randomly assigned 5 areas from $n=4$ mice per group were quantified. (J) Survival curves for control and Myf5Cre-Pcyt2 mice. $n=22$ mice per group. Data are shown as means \pm SEM. Each dot represents individual mice. ${ }^{*} p<$ $0.05, * * \mathrm{p}<0.01, * * * \mathrm{p}<0.001$, and $* * * * \mathrm{p}<0.0001$, n.s. not significant (unpaired Student $\mathrm{t}$ test, for survival analysis Mantel Cox test).

\section{Figure 3. Loss of Pcyt2 results in altered muscle membrane architectures.}

(A) Schematic diagram of GPMV isolation from primary myoblasts. (B) Polarization microscopy analysis of NR12S dye-stained GPMVs isolated from control and Myf5Cre-Pcyt2 myoblasts. GPMVs were isolated from cultured myoblasts from 2 mice per group. Representative images and quantifications are shown. Each dot represents GP values of a single GPMV. Scale bar $10 \mu \mathrm{m}$. (C) Schematic diagram and representative example of GPMVs (arrows) s immediately after isolation from skeletal myofibers. Images are from a wild type mouse at 0 and 30 minutes under GPMV conditions. Scale bar $50 \mu \mathrm{m}$. (D) Polarization microscopy of NR12S-stained GPMVs isolated from control and Myf5Cre-Pcyt2 primary myofibers (as shown in C). GPMVs were isolated from $\geq 100$ myofibers from 2 mice per group. Representative images and quantifications are shown. Each dot represents GP values of a single GPMV. Scale bar $10 \mu \mathrm{m}$. (E) Schematic set-up of Brillouin light scattering microscopy adapted to freshly isolated myofibers. (F) Surface stiffness analysis as measured by Brillouin 
frequency shift (BFS) from isolated control and Myf5Cre-Pcyt2 myofibers. Left panels indicate representative microscopy images. Each data point in the right panel represents a BFS peak value of the myofiber surface. $>15$ myofibers from $n=3$ mice per group were analyzed. (G) Representative qualitative membrane stiffness data of control and Myf5Cre-Pcyt2 myofibers as assessed by atomic force microscopy. As seen from the curve angles in the approach phase ( 0 to $-1000 \mathrm{~nm})$ and retraction phase $(-1000$ to $0 \mathrm{~nm})$, the cantilever bends much less for Myf5Cre-Pcyt2 myofibers, indicating lower surface stiffness. In the prolonged part of retraction phase ( 0 to $400 \mathrm{~nm}$ ) the cantilever remains deeper inserted within the Myf5CrePcyt2 myofibers, indicating a higher degree of surface deformation upon pressure. (H) Quantitative myofiber membrane stiffness as assessed by atomic force microscopy to quantify cell membrane stiffness using the Young's modulus scale (in kilopascal, kPa). For each myofiber we collected $\geq 4000$ measurements over a $5 \mu \mathrm{m}$ X $5 \mu \mathrm{m}$ area. Matlab's Randsample function was used to uniformly sample each myofiber measurements. Each dot represents 500 data points per each myofiber, from $n=20-26$ control and Myf5Cre-Pcyt2 myofibers. Data are shown as means \pm SEM. Each dot represents individual mice. ${ }^{*} p<0.05,{ }^{*} p<0.01,{ }^{* * *} p<$ 0.001 , and $* * * * p<0.0001$, n.s. not significant (unpaired Student t-test).

\section{Figure 4. Pcyt2 is essential for muscle membrane integrity and strain tolerance and improves muscle strength in aged mice}

(A) Penetrance of Evans blue into the quadriceps muscle of 6 month old control and Myf5CrePcyt2 mice after i.p. injection. Gross morphologies left) and histological sections are shown. Scale bars are $1 \mathrm{~cm}$ and $100 \mu \mathrm{m}$. (B) Quantification of Evans blue in the quadriceps muscle after extraction from the tissue determined $n=3$ per group. (C) Laser induced damage of myofibers isolated from 6 month old control and Myf5Cre-Pcyt2 mice. The extent of damage was followed over time using influx of the fm43. The injured membrane areas of the myofibers are indicated by two arrows. Right panel shows quantification of fm43 influx over the indicated time period +/- SEM; $n=9-12$ myofibers per group from two independent experiments. Scale bar $50 \mu \mathrm{m}$ (D) Running distance (in meters) during eccentric exercise of 6 month old control and Myf5Cre-Pcyt2 mice. Data are pooled from the first training bout on days 1-3 (4m min-1), the second training bout on days $4-11\left(9 \mathrm{~m} \mathrm{~min}^{-1}\right)$; and the third training bout on days 12-14 (20m min $\left.{ }^{-1}\right) \cdot n=4-6$ per group. (E) Histological analysis (H\&E staining) of 
quadriceps muscles isolated from 6 month old control and Myf5Cre-Pcyt2 mice, before (no training) and after (training) the eccentric exercise. Black arrows show inflammation migrating from a blood vessel; blue arrow indicates ectopic fat deposits. Scale bars $100 \mu \mathrm{m}$ (F) Myopathy scores in 6 month old control and Myf5Cre-Pcyt2 mice following eccentric exercise. The following parameters were used to calculate the score: inflammatory cell infiltrates, myofiber necrosis, myofiber atrophy, interstitial fibrosis, loss of sarcoplasmic membrane integrity, regenerating myofibers. Each was scored with 1-4 depending of the severity, and summed for the final value, $n=4-6$ mice per cohort. (G) Schematic diagram of muscle specific gene therapy on Pcyt2 deficient background. (H) Representative images, and grip strength quantification of control (saline) Myf5Cre-Pcyt2 (saline) and Myf5Cre-Pcyt2 (AAV6-CK8Pcyt2HA) mice (1) Pcyt2 activity in quadriceps muscles from young (6 month) and presarcopenic (24 month old) C57B6/J mice. Each dot represents individual mouse (J) PCYT2 activity in quadriceps muscle biopsied from young (20-30yr) and middle aged (45-62yr) healthy human volunteers. Each dot represents individual human (K) Schematic diagram and grip strength quantification of muscle specific gene therapy from 24 month old control (saline) and AAV6-CK8-Pcyt2HA transduced C57B6/J mice ( $\mathrm{n}=11-15$ per group). Data are shown as means \pm SEM. Each dot represents individual mice. ${ }^{*} p<0.05,{ }^{*} p<0.01,{ }^{*} *{ }^{*} p<$ 0.001 , and $* * * * p<0.0001$, n.s. not significant (unpaired Student t-test) 


\title{
Extended Data Figure legends
}

\section{Extended Data Figure 1. PE synthesis pathways and EPT1 rare disease mutation carriers.}

\begin{abstract}
(A) Schematic diagram of phosphatidylcholines (PC), phosphatidylethanolamines (PE) and phosphatidylserine (PS) phospholipids synthesis. EK-Ethanolamine kinase; PCYT2CTP:phosphoethanolamine cytidylyltransferase; EPT1- ethanolaminephosphotransferase 1; PSS2- Phosphatidylserine Synthase 2; PSD- Phosphatidylserine decarboxylase; CK- Choline kinase; $\quad$ PCYT1- Choline-phosphate cytidylyltransferase; $\quad$ CEPT1Choline/ethanolaminephosphotransferase 1; PSS1- Phosphatidylserine Synthase 1. (B) Height and weight gains of three patients carrying the homozygous missense variant c.335 G>C (p.Arg112Pro) in the EPT1 gene. Controls indicate WHO standards of median weights and heights at the respective ages +/- 2 standard deviations (SD).
\end{abstract}

\section{Extended Data Figure 2. Analysis of Pcyt2 deletion in mice.}

(A) Schematic diagram of exon 2 deletion in Myf5Cre-Pcyt2 mice and confirmation by RNA sequencing. Exon and introns structures as well as LoxP sites targeted to exon 2 and loss of exon 2 upon Cre-mediated recombination are shown for the murine Pcyt2 locus. $n=3$ animals per group.

\section{Extended Data Figure 3. Characterization of Myf5Cre-Pcyt2 mice.}

(A) Lipidomics analyses from quadriceps muscles isolated from 10 day old Myf5Cre-Pcyt2 and littermate control mice. Data are shown relative to control values. CE-cholesterol ester; CerCeramides; DAG-diacylglycerols; LPC-Iysophosphatidylcholines; LPElysophosphatidylethanolamines; PC-phosphatidylcholines; PE-phosphatidylethanolamines; PG-phosphatidylglycerols; PI-phosphatidylinositols; PS-phosphatidylserines; $\quad$ SMsphingomyelins; TAG-triacylglycerols. $n=4$ per group. (B) Detailed analysis of PE species with 
different chain lengths from quadriceps muscles of Myf5Cre-Pcyt2 as compared to control mice. (C) Body weights of control and Myf5Cre-Pcyt2 mice at P1 and P4. (D) Body length gains of control and Myf5Cre-Pcyt2 mice. $n=6$ per group for body length analysis. (E) Body weights of 2 months old control and Myf5Cre-Pcyt2 female mice. (F) Body lengths of 2 months old control and Myf5Cre-Pcyt2 female mice. (G) Skeletal muscle and tissue weight isolated from (G) 10 day and (H) 2 month old (P56) control and Myf5Cre-Pcyt2 littermate mice. QA, quadriceps; GC, gastrocnemius; TA, tibialis anterior muscles. Liver and spleen weights are shown as controls. $n=6-8$ per group Scale bars $1 \mathrm{~cm}$. (I-J) Gross skeletal muscle appearance of 56 days old control and Myf5Cre-Pcyt2 littermates. Data are shown as means \pm SEM. Each dot represents individual mice. ${ }^{*} p<0.05,{ }^{*} \mathrm{p}<0.01, * * * p<0.001$, and $* * * * p<0.0001$, n.s. not significant (unpaired Student t-test).

\section{Extended Data Figure 4. Early muscle morphology, myoblast fusion efficiency and hypertrophic muscle response in Myf5Cre-Pcyt2 mice.}

(A) Representative cross section of quadriceps isolated from 10 days old control and Myf5CrePcyt2 mice. (B) Representative images of BrDU labeled quadriceps from 2 days old control and Myf5Cre-Pcyt2 mice. Scale bar $60 \mu \mathrm{m}$. (C) Number of Pax7 positive nuclei in quadriceps from 6 months old control and Myf5Cre-Pcyt2 mice. Sections from 3 mice were examined, with $\geq 100$ nuclei per section counted. (D) Primary myoblast fusion assay. Myoblasts were isolated as depicted in methods section from $n=3$ mice per group. Each dot represents a calculated fusion index (number of fused nuclei/total number of nuclei) in triplicates, with a total of $\geq 300$ counted nuclei per one isolation. (E) Hypertrophic muscle growth in control and Myf5Cre-Pcyt2 mice. Following synergic ablation or sham surgery, M. plantaris weights were determined on the compensating limb. Data are shown as means \pm SEM. Each dot represents individual mice. n.s. not significant, $* p<0.05, * * p<0.01, * * * p<0.001$, and $* * * * p<0.0001$ (unpaired Student t-test). 


\section{Extended Data Figure 5. Muscle inflammation and metabolic assessment of Myf5Cre-Pcyt2 mice.}

(A) Representative electron microscopy images of quadriceps of 15 months old control and Myf5Cre-Pcyt2 mice. Note accumulation of tubular aggregates in the mutant animals (red arrows). Scale bar $2 \mu \mathrm{m}$. (B) Characterization of muscle inflammation in 12 months old Myf5Cre-Pcyt2 mice. Helper T cells (CD4+) and cytotoxic T cells (CD8+) are shown. Scale bar $100 \mu \mathrm{m}$ for H\&E stained and $50 \mu \mathrm{m}$ for immune cell staining. (C) Inflammatory cytokine levels in the quadriceps of 12 months old Myf5Cre-Pcyt2 mice. (D) Fed blood glucose levels on normal chow diet of 8 months old control and Myf5Cre-Pcyt2 mice. (E) Food consumption of chow diet fed 8 months old control and Myf5Cre-Pcyt2 mice. (F) Cage activity of 6 months old control and Myf5Cre-Pcyt2 mice ( $\mathrm{n}=12$ per group). Multiple ANOVA was used to analyze the data. (G-H) Energy expenditure of 6 months old control and Myf5Cre-Pcyt2 mice during the resting (light) (G) and active (dark) (H) phases. Multiple ANOVA was used to analyze the data. Data are shown as means \pm SEM. Each dot represents individual mice. ${ }^{*} p<0.05,{ }^{* *} p<0.01$, $* * * \mathrm{p}<0.001$, and $* * * * \mathrm{p}<0.0001$, n.s. not significant (unpaired Student t-test, unless otherwise stated).

\section{Extended Data Figure 6. Characterization of the brown adipose tissue from Myf5Cre-Pcyt2 mice.}

(A-B) Lipidomics analysis from brown fat isolated from 10-day old Myf5Cre -Pcyt2 and control mice. $n=4$ per group. (C) Brown fat differentiation in lipid free conditions from 2-day old primary pre-adipocytes isolated from control and Myf5Cre -Pcyt2 mice. Scale bar 50 $\mu$ m. (DE) Brown fat activity as addressed by exposure of 6-month-old control and Myf5Cre-Pcyt2 mice to cold (4C) or during fasting. (F) Ucp1 mRNA levels in brown fat of 6-month-old control and Myf5Cre-Pcyt2 mice. (G) Mitochondrial content in brown adipose tissue. (H) BAT mitochondrial structure of 6-month-old Myf5Cre-Pcyt2 mice. Scale bar $1 \mu \mathrm{m}$. (I-J) Complex I and II activities of brown fat mitochondria. Paired Student t-test was used to analyze the data. Data are shown as means \pm SEM. Each dot represents individual mice. ${ }^{*} p<0.05,{ }^{* *} p<0.01$, 
$* * * p<0.001$, and $* * * * p<0.0001$, n.s. not significant (unpaired Student t-test, unless otherwise stated)

\section{Extended Data Figure 7. Specific inactivation of Pcyt2 in multiple mouse} tissues.

(A) Schematic diagram to generate adipose tissue specific Pcyt2 deficient mice (AdipoQCrePcyt2).(B) Body weights and appearances of 6 months old control and AdipoQCre-Pcyt2 mice. (C) Fasting blood glucose of 6 months old control and AdipoQCre-Pcyt2 littermates fed a chow diet. (D) Schematic diagram of motor neuron specific Pcyt2 deficient mice (Mnx1Cre-Pcyt2). (E) Body weights of 8 months old control and Mnx1Cre-Pcyt2 mice. (F) Absence of any overt clasping behavior and appearance in 8 months old Mnx1Cre-Pcyt2 mice. (G) Schematic diagram of intestine epithelium specific Pcyt2 deficient mice (VilinCre-Pcyt2). (H) Body weights of 6 months old control and VilinCre-Pcyt2 littermates. (I) Histological sections of intestine isolated from 12 months old control and VilinCre-Pcyt2 mice. (J) Schematic diagram of skin epithelium Pcyt2 deficient mice (K14Cre-Pcyt2). (K) Body weights and appearances of 6 months old control and K14Cre-Pcyt2 mice. (L) Histological sections of intestine isolated from 12 months old control and K14Cre-Pcyt2 littermates. (M) Schematic diagram of mature muscle specific Pcyt2 deficient mice (MCKCre-Pcyt2). (N) Grip strength of 18 months old control and muscle specific MckCre-Pcyt2 mice. Each dot represents individual mice, each mouse was tested in triplicates. Mean values \pm SEM are displayed. ${ }^{*} p<0.05,{ }^{* *} p<0.01$, $* * * p<0.001$, and ${ }^{* * * *} \mathrm{p}<0.0001$, n.s. not significant (unpaired Student t-test).

\section{Extended Data Figure 8. Assessment of calcium handling, mitochondrial respiration and oxidative stress in skeletal muscle.}

(A) Voltage-dependence of the peak rate of sarcoplasmatic reticulum (SR) $\mathrm{Ca}^{2+}$ release (d[CaTot]/dt) measured from rhod-2 $\mathrm{Ca}^{2+}$ transients in fibers from control and Myf5Cre-Pcyt2 mice. $n=23-22$ fibers isolated from $n=5-6$ mice per group. (B) Decline of voltage-activated fluo4FF Ca ${ }^{2+}$ transients in control and Myf5Cre-Pcyt2 muscle fibers in response to an exhausting 
voltage stimulation protocol. $n=8-6$ fibers isolated from $n=2-3$ mice per group. (C) LC3 I/II levels in quadriceps from 8 months old control and Myf5Cre-Pcyt2 mice. (D-E) Ultrastructure and total numbers of muscle mitochondria from 8 months -old control and Myf5Cre-Pcyt2 mice. Scale bar $1 \mu \mathrm{m}$. (F-G) Mitochondrial function measured by complex I and II activity from muscles from 6 months old control and Myf5Cre-Pcyt2 mice. Paired Student t-test.. (H) Evidence of increased protein oxidative damage in quadriceps muscles isolated from 6 months old Myf5Cre-Pcyt2 mice. Representative blot are shown for $\mathrm{n}=3$ mice per group. (I) Catalase anti-oxidant activity in quadriceps muscles from 6 months old control and Myf5CrePcyt2 mice. (J) Increased levels of phospho-JNK (pJNK) and FoxO1 in quadriceps muscles from 6 months old Myf5Cre-Pcyt2 mice as compared to controls. $\mathrm{n}=3$ per group. (K) Increased levels of myofiber wasting markers in muscles of 8 months old Myf5Cre-Pcyt2 mice. Data are shown as means \pm SEM. Each dot represents individual mice. ${ }^{*} p<0.05, * * p<0.01,{ }^{*} * p<0.001$, and $* * * * \mathrm{p}<0.0001, \mathrm{n} . \mathrm{s}$. not significant (unpaired Student t-test, unless otherwise stated).

\section{Extended Data Figure 9. Actin organization and Pax7+ progenitor numbers after eccentric exercise in Myf5Cre-Pcyt2 mice.}

(A) Relative change in skeletal muscle weights from untrained and trained (3 bouts of eccentric exercise) 6 month old control and Myf5Cre-Pcyt2 mice. QA, quadriceps; GC, gastrocnemius; TA, tibialis anterior muscles. $n=4-9$ per group. (B) Tissue fibrosis (collagen staining) of quadriceps muscles isolated from 6 month old control and Myf5Cre-Pcyt2 mice, before (no training) and after (training) the eccentric exercise. Black arrows indicate collagen deposits. Scale bars $100 \mu \mathrm{m}$ (C) Dysferlin immunohistochemistry staining of the quadriceps muscles before and after eccentric exercise. DAPI was used to image nuclei. Scale bar $25 \mu \mathrm{m}$ (D) F-actin staining of skeletal muscle tissue isolated from 6 months old control and Myf5CrePcyt2 mice after eccentric exercise. Images of quadriceps cross-sections were taken using 20x magnifications. $\geq 100$ myofibers were counted. $n=3$ mice per group. Scale bar $25 \mu m$. (E) Number of Pax $7^{+}$nuclei in quadriceps muscles from 6 months old control and Myf5Cre-Pcyt2 mice following eccentric exercise. Sections from 4 mice per group were examined, with $\geq 100$ nuclei per section counted. Data are shown as means \pm SEM. Each dot represents individual 
mice. ${ }^{*} p<0.05,{ }^{* *} p<0.01,{ }^{* * *} p<0.001$, and ${ }^{* * * *} p<0.0001$, n.s. not significant (unpaired Student t-test)

\section{Extended Data Figure 10. Reduced Pcyt2 activity and levels in muscle ageing.}

(A) Grip strength of control (saline), Myf5Cre-Pcyt2 (saline) and Myf5Cre-Pcyt2 (AAV6 CK8 Pcyt2 HA) injected mice. Each dot represents individual mice. (B) Pcyt2 mRNA levels in quadriceps muscles from young (6 month) and pre-sarcopenic (24 month old) C57B6/J mice. Each dot represents individual mouse (C) PCYT2 activity/age correlations and (D) mRNA levels in quadriceps muscle biopsied from young (20-30yr) and middle aged (45-62yr) healthy human volunteers. Each dot represents individual human (E) Assessment of Pcyt2HA expression as determined by anti-HA immunoprecipitation, followed by an anti-Pcyt2 blot, from quadriceps muscles isolated 2 months after the gene delivery. Equal protein input is shown in the Ponceau S stained gel. $\mathrm{n}=6$ (control) or 5 (Pcyt2-HA) mice per treatment group. (F) Grip strength of 24 month old control (saline) and AAV6-CK8-Pcyt2HA transduced C57B6/J mice. Each dot represents individual mice. Data are shown as means \pm SEM. ${ }^{*} p<0.05,{ }^{*} p<$ $0.01, * * * p<0.001$, and $* * * * p<0.0001$, n.s. not significant (unpaired Student t-test)

\section{Extended Data Video legends}

\section{Extended Data Video 1-4}

Laser induced myofiber damage assessment on myofibers isolated from 6-month-old control and Myf5Cre-Pcyt2 mice. Videos are from 2 independent isolations.

\section{Extended Data Video 5}


Video of the last bout of eccentric exercise (day 13) of 6-month-old control and Myf5CrePcyt2 mice. First, mice were adjusted to a lower speed (9m $\left.\mathrm{min}^{-1} ; \mathrm{t}=0-10 \mathrm{~s}\right)$, followed by a higher speed bout (20 m min-1; $\mathrm{t}=10-40 \mathrm{~s})$.

\section{Methods}

\section{CONTACT FOR REAGENT AND RESOURCE SHARING}

Further information and requests for resources and reagents should be directed to and will be fulfilled by the Lead Contacts Josef M. Penninger (josef.penninger@ubc.ca) or Domagoj Cikes (domagoj.cikes@imba.oeaw.ac.at).

\section{METHOD DETAILS}

\section{Studies in Humans}

Patients with PCYT2 and EPT1 deficiency were identified previously ${ }^{7,9}$. Their height and weight were recorded at visits to the hospital. The spinal MRI was performed at the age of 19 years. All data presented are being shared with parental and patient consent.

\section{Human biopsies}

All human experiments were approved by the regional ethical review board in Stockholm (2014/516-31/2 and 2010/786-31/3) and complied with the Declaration of Helsinki. Oral and written informed consent were obtained from all subjects prior to participation in the study. 8 healthy young adults (age 21-29) and 8 middle-aged (age 45-62) subjects were recruited. The subjects did not use any medications and were nonsmokers. Biopsies of the quadriceps vastus lateralis muscle were obtained under local anesthesia using the Bergström percutaneous needle biopsy technique ${ }^{79}$. The biopsies were immediately frozen in isopentane, cooled in liquid nitrogen, and stored at $-80^{\circ} \mathrm{C}$ until further analysis.

\section{Studies in zebrafish}




\section{Generation of mutant zebrafish and analysis}

Pcyt2 mutant zebrafish at F0 have been described previously ${ }^{7}$. For histological examination of zebrafish models, animals were sacrificed by lethal anesthesia with buffered tricaine methanesulfonate. After gross examinations, their abdomen was opened and whole body was immersed in $4 \%$ paraformaldehyde (PFA) for $72 \mathrm{hr}$. After fixation, the animal was cut with a surgical blade into $0.5 \mathrm{~mm}$ pieces and embedded in paraffin blocks. $3 \mu \mathrm{m}$ sections were further processed for routine hematoxylin and eosin staining. Back muscle cross sectional areas of the same anatomical region was imaged under 10X magnification, followed by analysis (75-80 myofibers; $n=4$ animals per group) with ImageJ software.

\section{Studies in mice}

\section{Mouse lines and diets}

All mice were housed in the IMBA mouse colony with a $12 \mathrm{~h} \mathrm{light/dark} \mathrm{cycle} \mathrm{in} \mathrm{a} \mathrm{temperature-}$ controlled environment and fed a standard chow diet. Pcyt2 conditional mice have been described previously ${ }^{80}$. In all cases, all mice described in our experiments were littermates, matched for age and sex. Tissue specific Pcyt2 mutant mice were generated by establishing a colony of Pcyt $2^{\text {flox/flox }}$ mice crossed Cre transgenic mice. Villin Cre mice originate from Institut Curie (Sylvie Robine Lab). The following mouse lines were obtained from the Jackson Laboratory (Jackson Lab, Bar Harbor, US): Adipoq Cre (B6;FVB-Tg(Adipoq-cre)1Evdr/J); Alb Cre (B6.Cg-Speer6-ps1Tg(Alb-cre)21Mgn/J); Mck Cre (B6.FVB(129S4)-Tg(Ckmm-cre)5Khn/J. All animal experiments were approved by the Animal Care and Use Committee at IMBA.

\section{Functional in vivo muscle tests}

Grip strength: Two, 4 and 6-month old mice (control and Pcyt2 Myf5 KO mice) were subjected to grip strength tests using a grip strength meter (Bioseb, USA), following standardized operating procedures. Prior to tests, mice were single caged for two weeks, in order to avoid any littermate influence on their performance. Each mouse was tested three times, with a 15minute inter-trial interval, and values were averaged among the three trials. Clasping index 
was evaluated as described previously ${ }^{81}$. Each mouse was scored three times, and an average of scores was calculated.

Eccentric exercise: Treadmill training was performed as previously described ${ }^{82}$. Briefly, single caged mice were acclimatized for treadmill exercise for three days on low speed $\left(4 \mathrm{~m} \mathrm{~min}^{-1}\right.$ for 40 min per day), followed by a 7-day training on a medium speed ( $4 \mathrm{~m} \mathrm{~min}^{-1}$ for 40 min plus $9 \mathrm{~m} \mathrm{~min}^{-1}$ for $20 \mathrm{~min}$ per day), and a 2-day stress training (20m min-1 for $20 \mathrm{~min}$ per day) to test endurance under stress conditions. Immediately after the completion of the exercise, mice were sacrificed, and muscles were collected for histological analysis.

Synergic muscle ablation: For synergic ablation experiments, all surgical procedures were performed under aseptic conditions with the animals deeply anaesthetized with pentobarbital sodium (60 mg/kg i.p.). Compensatory overload of the plantaris muscle was performed unilaterally via removal of the major synergistic muscles (gastrocnemius-medialis, -lateralis and soleus), as described previously ${ }^{83}$. A sham operation was systematically performed on the control hindlimb, which consisted of separating tendons of the soleus and gastrocnemius muscles from the plantaris muscle. Analgesic was administered to the animals for two days following the operation. The overload lasted for 14 days. For maintaining the activity of the animals during the overload period, moderate speed walking training was used on a treadmill (10 degree ascents, 4-5 m/min, $30 \mathrm{~min}, 6$ times/week). At the end of this period, after animal sacrifice, the plantaris muscle was removed bilaterally, trimmed of excess fat and connective tissue, and wet weighed and processed for further analysis.

\section{AAV-based vector delivery}

For the AAV6 treatment, Pcyt2 (NM_024229.3) was C-terminally tagged with an HA-tag and cloned into the AAV6-CK8 muscle specific expression vector ${ }^{62}$ using the Sal1-Kpn1 restriction sites. AAV6 viral particles were prepared as previously described ${ }^{84}$. For gene therapy of Myf5Cre-Pcyt2 mutant mice, 4 day old pups were injected i.p. with $2 \times 10^{11}$ vector genomes per mouse. For ageing studies 24-month-old C57B6/J mice (Jackson Labs, Bar Harbor) were single caged for two weeks for acclimatization. Grip strength was measured before AAV6 delivery using the grip strength meter. On the day of the AAV injection, mice were 
anesthetized with isoflurane, and injected retro-orbitally either with AAV6-Pcyt2HA $\left(5 \times 10^{12}\right.$ vector genomes per mouse) or as a control saline. Expression of the Pcyt2-HA was determined by Western blotting and grip strength was measured one and two months after the injection.

\section{Metabolic studies}

Animals were fed standard chow diet and blood glucose was measured at fed and fasted state (16h fasting). Standard chow diet was purchased from SSNIFF (V 1184-300; 10mm pellets autoclavable; $49 \% \mathrm{kcal}$ carbohydrates, $36 \% \mathrm{kcal}$ protein and $15 \% \mathrm{kcal}$ fat). Measurements were done at the same time of the day, by collecting blood samples after tail snipping and applying the blood samples to Onetouch Verio strips (LifeScan, GmbH). Food consumption was measured on single cage housed animals over a period of two weeks, after acclimatization single caging for one week. For calorimetry, measurements were performed at room temperature $(21 \mathrm{C}-23 \mathrm{C})$ on a $12 / 12 \mathrm{~h}$ light/dark cycle in a PhenoMaster System (TSEsystems, Bad Homburg, Germany) using an open circuit calorimetry system ${ }^{85}$. Mice (4-7 months old) were housed individually, trained on drinking nozzles for 7 days and allowed to adapt to the PhenoMaster chamber for 2 days. Food and water were provided ad libitum. Parameters of indirect calorimetry and activity were determined for 5 consecutive days. Body weights were recorded at the beginning and end of the experiments and average values were plotted against energy expenditure and activity ${ }^{86}$. To address brown fat activity, 6-month-old mice were housed at $4^{\circ} \mathrm{C}$ and body temperature was measured during the fed period using a thermometer for small animals (Thermometer TK 98802; Bioseb). Temperature was recorded every hour over a $4 \mathrm{~h}$ period. After a 2 day recovery period at room temperature, the same mice were fasted and the body temperatures determined at 6 and 16 hours of fasting.

\section{Primary cell culture conditions}


Myoblasts: Primary myoblasts were isolated as previously described ${ }^{87}$. Briefly, after collagenase (type 2; $500 \mathrm{U}$ per $\mathrm{mL}$ ) and liberase digestion $\left(2.5 \mathrm{U} / \mathrm{mL}\right.$ ) for $1.5 \mathrm{hr}$ on $37^{\circ} \mathrm{C}$, the tissue slurry was seeded directly on Matrigel coated dishes $(45 \mu \mathrm{g} / \mathrm{cm} 2)$ in DMEM/F12 containing 20\%FCS (Sigma-Aldrich) and 10\% horse serum (Sigma-Aldrich) and penicillinstreptomycin (Life Technologies, final conc., $50 \mathrm{U} / \mathrm{mL}$ of penicillin). Removal of fibroblasts was achieved by serial (2-4) passaging and pre-plating the cell suspension on collagen Type 1 coated dishes for $1 \mathrm{hr}$ prior transfer to Matrigel coated dishes $(4.5 \mu \mathrm{g} / \mathrm{cm} 2)$. Differentiation into myotubes was done by culturing myoblasts in serum/lipid free DMEM/F12 with InsulinTransferrin-Selenium supplement (ThermoFisher, $\mathrm{GmbH}$ ) for 5 days.

Brown adipocytes: Primary brown adipocyte differentiation was done as previously described 88. Briefly, after harvesting from 2-day old pups, brown fat tissue was digested in DMEM collagenase solution (type 2; $500 \mathrm{U}$ per $\mathrm{mL}$ ) for $1.5 \mathrm{hr}$. Cells were grown in DMEM with $10 \%$ FCS and penicillin-streptomycin (Life Technologies, final conc., $50 \mathrm{U} / \mathrm{mL}$ of penicillin). Differentiation into mature brown fat adipocytes was achieved by adding an adipogenic cocktail $(0.5 \mu \mathrm{g} / \mathrm{mL}$ insulin, $5 \mu \mathrm{M}$ dexamethasone, $1 \mu \mathrm{M}$ rosiglitazone, and $0.5 \mathrm{mM}$ IBMX) in serum free media for 3 days, following maintenance in serum free media supplemented only with insulin for another 4 days. Lipid droplets were visualized with Bodipy 493/503 solution (ThermoFisher).

\section{Lipidomics}

Quadriceps muscles and brown adipose tissues were isolated from 10-day old pups and snap frozen in liquid nitrogen. Muscle tissue was homogenized using a Precellys 24 tissue homogenizer (Precellys CK14 lysing kit, Bertin). Per mg tissue, $3 \mu \mathrm{L}$ of methanol were added. $20 \mu \mathrm{L}$ of the homogenized tissue sample was transferred into a glass vial, into which $10 \mu \mathrm{L}$ internal standard solution (SPLASH ${ }^{\circledR}$ Lipidomix $^{\circledR}$, Avanti Polar Lipids) and $120 \mu \mathrm{L}$ methanol were added. After vortexing, $500 \mu \mathrm{L}$ Methyl-tert-butyl ether (MTBE) were added and incubated in a shaker for $10 \mathrm{~min}$ at room temperature. Phase separation was performed by adding $145 \mu \mathrm{L}$ MS-grade water. After $10 \mathrm{~min}$ of incubation at room temperature, samples were centrifuged at $1000 \mathrm{xg}$ for $10 \mathrm{~min}$. An aliquot of $450 \mu \mathrm{L}$ of the upper phase (organic) was collected and dried in a vacuum concentrator. The samples were reconstituted in $200 \mu \mathrm{L}$ 
methanol and used for LC-MS analysis. The LC-MS analysis was performed using a Vanquish UHPLC system (Thermo Fisher Scientific) combined with an Orbitrap Fusion ${ }^{\mathrm{TM}}$ Lumos $^{\mathrm{TM}}$ Tribrid $^{\mathrm{TM}}$ mass spectrometer (Thermo Fisher Scientific). Lipid separation was performed by reversed phase chromatography employing an Accucore C18, $2.6 \mu \mathrm{m}, 150 \times 2 \mathrm{~mm}$ (Thermo Fisher Scientific) analytical column at a column temperature of $35^{\circ} \mathrm{C}$. As mobile phase A we used an acetonitrile/water (50/50, v/v) solution containing $10 \mathrm{mM}$ ammonium formate and $0.1 \%$ formic acid. Mobile phase B consisted of acetonitrile/isopropanol/water (10/88/2, $\mathrm{v} / \mathrm{v} / \mathrm{v}$ ) containing $10 \mathrm{mM}$ ammonium formate and $0.1 \%$ formic acid. The flow rate was set to $400 \mu \mathrm{L} / \mathrm{min}$. A gradient of mobile phase B was applied to ensure optimal separation of the analysed lipid species. The mass spectrometer was operated in ESI-positive and -negative mode, capillary voltage $3500 \mathrm{~V}$ (positive) and $3000 \mathrm{~V}$ (negative), vaporize temperature $320^{\circ} \mathrm{C}$, ion transfer tube temperature $285^{\circ} \mathrm{C}$, sheath gas 60 arbitrary units, aux gas 20 arbitrary units and sweep gas 1 arbitrary unit. The Orbitrap MS scan mode at 120000 mass resolution was employed for lipid detection. The scan range was set to $250-1200 \mathrm{~m} / \mathrm{z}$ for both positive and negative ionization mode. The AGC target was set to $2.0 \mathrm{e} 5$ and the intensity threshold to 5.0e3. Data analysis was performed using the TraceFinder software (ThermoFisher Scientific). The lipidomics results from five biological replicates per group were analyzed as reported previously ${ }^{89}$. Briefly, the amount of each lipid was normalized to the sum of concentrations for all lipid species, measured in a single biological replicate. Values were next averaged over the five biological replicates for control and Myf5Cre-Pcyt2 samples, log2 transformed, and compared between the groups. To determine significant changes un-paired t-test was used and represented as ns - non significant, ${ }^{*} P<0.05,{ }^{* *} P<0.01,{ }^{* * *} P<0.001,{ }^{* * * *} P<0.0001$.

\section{Respiration and catalase activity measurements}

Mitochondrial respiratory parameters were measured with high-resolution respirometry (Oxygraph-2k, Oroboros Instruments, Innsbruck, Austria). Briefly, isolated mitochondria (0.03 $\mathrm{mg} / \mathrm{mL}$ ) were incubated in a buffer containing $80 \mathrm{mM} \mathrm{KCl}, 5 \mathrm{mM} \mathrm{KH_{2 }} \mathrm{PO}_{4} \mathrm{mM}, 50 \mathrm{mM}$ Mops, $1 \mathrm{mM}$ ethylene glycol-bis(2-aminoethylether)- $\mathrm{N}, \mathrm{N}, \mathrm{N}^{\prime}, \mathrm{N}^{\prime}$-tetraacetic acid, and $1 \mathrm{mg} / \mathrm{mL}$ fatty acid-free bovine serum albumin $\left(\mathrm{pH} 7.4,37^{\circ} \mathrm{C}\right) 1$. Respiration was initiated either by the addition of $5 \mathrm{mM}$ glutamate and $5 \mathrm{mM}$ malate (complex I), $10 \mathrm{mM}$ pyruvate (complex I), or $10 \mathrm{mM}$ succinate in the presence of $1.4 \mu \mathrm{M}$ rotenone (complex II). Transition to State-3 
respiration was induced by the addition of $1 \mathrm{mM}$ adenosine diphosphate. Maximum electron transfer system capacity was measured by titration of carbonyl cyanide-4-(trifluoromethoxy) phenylhydrazone in steps of $0.1 \mu \mathrm{M}$. Addition of exogenous cytochrome $\mathrm{c}(2.5 \mu \mathrm{M})$ was used to estimate the permeability of the outer mitochondrial membrane as a quality control. Oxygen consumption rates were obtained by calculating the negative time derivative of the measured oxygen concentration. Catalase activity was determined from isolated quadriceps of 6-month-old mice using a catalase activity assay according to the manufacturer recommendations (\#700910, Cayman Chemical).

\section{Isolation and imaging of Giant Plasma Membrane Vesicles (GPMVs)}

GPMVs were prepared as previously described ${ }^{90}$. Briefly, myoblasts were seeded on a $60 \mathrm{~mm}$ petri dish until 70\% confluency. Before GPMV formation, they were washed twice with GPMV buffer (150 mM NaCl, $10 \mathrm{mM}$ Hepes, $2 \mathrm{mM} \mathrm{CaCl}_{2}, \mathrm{pH} 7.4$ ) and finally $2 \mathrm{ml}$ of GPMV buffer was added to the cells together with 25 mM PFA and 10 mM DTT (final concentrations). After incubation for $2 \mathrm{hr}$ at $37^{\circ} \mathrm{C}$, GPMVs were collected from the supernatant. For GMPV preparation from myofibers, the Extensor digitorum longus (EDL) muscle was digested in collagenase supplemented medium (type $1,2 \mathrm{mg} / \mathrm{mL}$ ) for $2.5 \mathrm{hr}$ at $37^{\circ} \mathrm{C}$, followed by trituration and single myofiber isolation as described above. Myofibers were then gently washed twice with FCS free DMEM, followed by a brief 1 min wash with GPMV buffer containing 25 mM PFA, to prevent myofiber hypercontraction. Finally, GPMV buffer with $25 \mathrm{mM}$ PFA and $10 \mathrm{mM}$ DTT was added to the myofibers. After incubation for $2 \mathrm{hr}$ at $37^{\circ} \mathrm{C}, \mathrm{GPMVs}$ were collected from the supernatant. GMPVs were labelled with the polarity sensitive membrane probe NR12S (a kind gift from A. Klymchenko) at $0.1 \mu \mathrm{g} / \mathrm{ml}$ final probe concentration in phosphate buffered saline (PBS) for 5 minutes and then immediately imaged at room temperature. Spectral imaging was performed on a Zeiss LSM 780 confocal microscope equipped with a 32-channel GaAsP detector array. Laser light at $488 \mathrm{~nm}$ was used for fluorescence excitation of NR12S. The lambda detection range was set between 490 - $691 \mathrm{~nm}$. Images were saved in Ism file format and then analyzed by using a custom plug-in compatible with Fiji/ImageJ3 ${ }^{91}$. Glass coverslips (thickness of $0.17 \mathrm{~mm}$ ) were used to measure generalized polarization which reflects 
membrane lipid packing/order using the following formula where $I_{560}$ and $I_{650}$ are the fluorescence intensities at $560 \mathrm{~nm}$ and $650 \mathrm{~nm}$ respectively:

$$
G P=\frac{I_{560}-I_{650}}{I_{560}+I_{650}}
$$

\section{Brillouin Microscopy of myofibers}

Brillouin Light Scattering Microscopy (BLSM) was performed using an inverted confocal sample-scanning microscope with a Brillouin imaging-spectrometer as described by us previously 55,92 . Briefly the setup employed a $532 \mathrm{~nm}$ single-frequency probing laser and is based on a 2-stage cross dispersion Virtual Imaged Phase Array (VIPA) with intermediate Fourier and image plane filtering, a cooled EM-CCD camera (Hamamatsu ImagEMII) for detection ${ }^{93,94}$, and can achieve a spectral finesse $>85$. For more scattering samples, we also employed a heated lodine absorption cell in the detection path tuned to the laser wavelength to reduce the elastic scattering signal ${ }^{95}$. To generate spatial maps, samples were scanned with a 3-axis piezo electric stage (Physik Instrumente). Imaging was performed through 1.3 Numerical Aperture (NA) Si immersion-oil objective (Olympus) and confocallity was assured via a physical pinhole of $\sim 1$ Airy Unit (AU) before coupling the light into the spectrometer. Widefield transmitted light images were used to determine the scanning area for each sample. Several cross-sectional scans were performed for each myofiber at positions separated by a 1 ? $\mathrm{m}$. Acquisition was controlled by a custom Labview based script developed by the company THATec. The acquisition (dwell) time per voxel was $100 \mathrm{~ms}$ and the power measured at the sample was 2-3mW. Each measured spectra was de-convolved with the complete system spectral response as determined for the attenuated elastic scattering peak measured prior to each scan in the same sample. Prior and subsequent to each imaging session the spectra of water and ethanol were measured on a separate imaging arm and used for calibrating the spectral projection based on paraxial theory ${ }^{96}$, adapted to a dual VIPA setup. Each spectrum was then analyzed in Matlab (Mathworks) using custom developed scripts employing Spectral Phasor Analysis ${ }^{97}$ Results were confirmed to be in agreement with ones obtained from conventional non-linear least-squares fitting of deconvolved spectra consisting of the Stokes and Anti-Stokes Brillouin peaks using Lorentzian functions, however 
yielding lower uncertainties on average.. The extracted Brillouin peak frequency, which scales with the local elastic storage modulus, is taken to be indicative of the local stiffness. All measurements were performed at $37^{\circ} \mathrm{C}$ and $5 \% \mathrm{CO} 2$.

\section{Atomic force microscopy}

Force spectroscopy by means of Atomic force microscopy was done on individual myofibers after isolation from EDL muscles. Fibers were either cultured on matrigel coated dishes (QI mode) or probed immediately after isolation (Force spectroscopy). A JPK (Bruker) Nanowizard4 AFM atomic force microscope was used for the AFM experiments. This system implements the Quantitative Imaging (QI) mode, where a force-curve is acquired at each pixel location of the imaged area, providing stiffness data together with topography. The QP-BioAC cantilevers from Nanosensors $(0.06 \mathrm{~N} / \mathrm{m}$, less than $10 \mathrm{~nm}$ nominal tip radius) were used because of their ability to work in QI mode with biological samples. The approach and retract speeds were kept constant at 52 microns/s. The model used to obtain the Young's modulus from the acquired data was Hertz/Sneddon, as implemented in the JPK software. The paraboloid model was chosen as the most suitable for the sharp tips of the QP-BioAC cantilevers. For the fits forces up to $60 \mathrm{pN}$ were considered, where indentation depths remained below $500 \mathrm{~nm}$. Each of the fibers retrieved $\geq 4000$ values $(5 \mu \mathrm{m} \times 5 \mu \mathrm{m}$ sampled in $64 \times 64$ data pixels). Matlab's Randsample function was used to uniformly sample 500 data points.

For the force curves acquired for the qualitative comparison, forces up to $600 \mathrm{pN}$ were applied to a single location each, using same cantilevers and approach speeds. Samples were kept at a $37^{\circ} \mathrm{C}$ during the experiment.

\section{Laser-induced damage of myofiber cell membranes}

Myofibers were freshly isolated from EDL muscle of 4-month old mice by digestion in collagenase supplemented media (type $1,2 \mathrm{mg} / \mathrm{ml}$, Sigma) for $2 \mathrm{hr}$ at $37^{\circ} \mathrm{C}$. After digestion, individual fibers were transferred onto a 4-well chamber slide (Nunc Lab-Tek, Merck, GmbH)

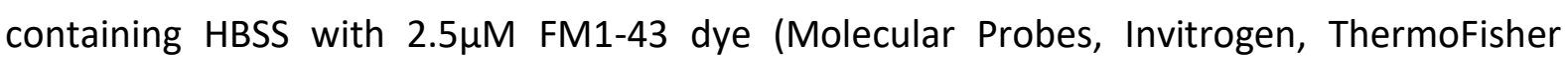
Scientific, $\mathrm{GmbH}$ ). Laser-induced cell membrane damage was performed as previously 
described ${ }^{58}$. Briefly, a $5 \times 5$ pixel area of the plasma membrane was exposed to a Laser at $20 \%$ of maximum power (Enterprise, $80 \mathrm{~mW}, 351 / 364 \mathrm{~nm}$ ) for $5 \mathrm{~s}$ using a Zeiss-LSM 510 confocal microscope equipped with a $\times 63$ water immersion lens (N.A. 1.3). Following the laser damage, distribution of the FM1-43 dye was imaged using high speed video captures. Throughout the experiment, cells were kept at a $37^{\circ} \mathrm{C}$ in a $5 \% \mathrm{CO}_{2}$ chamber.

\section{Ca2+ dynamics under voltage-clamp protocol}

The flexor digitorum brevis (FDB) and interosseous (IO) muscles were freshly isolated from mice and incubated with collagenase (Sigma type 1) for $1 \mathrm{hr}$ at $37^{\circ} \mathrm{C}$ in a Tyrode solution. Single myofibers were isolated by gentle mechanical trituration of the collagenase-treated muscles within the experimental chamber. Fibers were voltage-clamped using the siliconevoltage-clamp technique as described (Jacquemond, 1997; Lefebvre et al., 2014) with the voltage-clamp pipette filled with a solution containing (in mM) $140 \mathrm{~K}$-glutamate, 5 Na2-ATP, $5 \mathrm{Na} 2$-phosphocreatine, $5.5 \mathrm{MgCl}$ 2, 5 glucose, 5 HEPES and either $15 \mathrm{EGTA}, 6 \mathrm{CaCl}$, and 0.1 rhod-2 or 0.1 fluo-4FF. The extracellular solution contained (in mM) 140 TEA-methanesulfonate, $2.5 \mathrm{CaCl} 2,2 \mathrm{MgCl}$, 1 4-aminopyridine, $10 \mathrm{HEPES}$ and 0.002 tetrodotoxin. For fluo4FF fluorescence measurements, the extracellular solution also contained $0.05 \mathrm{mM} N$-benzylp-toluene sulfonamide (BTS) to block contraction. All solutions were at pH 7.2. Voltage-clamp and membrane current measurements were done with an RK-400 patch-clamp amplifier (BioLogic, Claix, France) in whole-cell voltage-clamp configuration. Command voltage pulse generation was achieved with an analog-digital converter (Digidata 1440A, Axon Instruments, Foster City, CA) controlled by pClamp 9 software (Axon Instruments). Analog compensation was adjusted to decrease the effective series resistance. Holding voltage was always set to $80 \mathrm{mV}$. Following insertion of the micropipette extremity into the muscle fiber, a $30 \mathrm{~min}$-long period of equilibration was allowed before taking measurements. Rhod-2 and fluo-4-FF fluorescence were detected with a Zeiss LSM 5 Exciter confocal microscope equipped with a $63 \times$ oil immersion objective (numerical aperture 1.4). For detection of rhod-2 and fluo-4 FF fluorescence, excitation was from the $543 \mathrm{~nm}$ line of a HeNe laser and from the $488 \mathrm{~nm}$ line of an Argon laser, respectively, and fluorescence was collected above $560 \mathrm{~nm}$ and above 505 $\mathrm{nm}$, respectively. Both rhod- 2 and fluo-4 FF voltage-activated fluorescence changes were 
imaged using the line-scan mode $(x, t)$ of the system and expressed as F/FO where F0 is the baseline fluorescence. Rhod-2 Ca2+ transients were triggered by $0.5 \mathrm{~s}$-long depolarizing pulses of increasing amplitude. From these, the rate of SR calcium release (d[CaTot]/dt) was calculated as described (Kutchukian et al., 2016; Lefebvre et al., 2014). Fluo-4-FF was used under non-EGTA buffering intracellular conditions to assess the resistance of the fibers to a fatigue protocol. For this, fibers were stimulated by consecutive trains of thirty $5 \mathrm{~ms}$-long pulses from $-80 \mathrm{mV}$ to $+60 \mathrm{mV}$ delivered at $100 \mathrm{~Hz}$ : 40 trains were applied, separated by a 0.7 s interval.

\section{Immunoblotting and Elisa measurements}

Western blotting: For immunoblotting analyses, tissues were snap frozen and homogenized in RIPA buffer (Sigma; R0278) containing Halt protease and phosphatase inhibitor cocktail (Thermo Scientific; 78440) immediately after isolation from sacrificed animals. Protein levels were determined using the Bradford assay kit (Pierce, $\mathrm{GmbH}$ ) and lysates containing equal amounts of protein were subjected to SDS-PAGE, further transferred to nitrocellulose membranes. Western blotting was carried out using standard protocols. Blots were blocked for 1 hour with 5\% BSA (Sigma Aldrich; \#820204) in TBST (1× TBS and 0.1\% Tween-20) and were then incubated overnight at $4^{\circ} \mathrm{C}$ with primary antibodies diluted in $5 \%$ BSA in TBST (1:250 dilution). Blots were washed three times in TBST for $15 \mathrm{~min}$, then incubated with HRPconjugated secondary antibodies diluted in 5\% BSA in TBST (1:5000 dilution) for $45 \mathrm{~min}$ at room temperature, washed three times in TBST for $15 \mathrm{~min}$ and visualized using enhanced chemiluminescence (ECL Plus, Pierce, 1896327). The following primary antibodies were used: anti-phospho-JNK (\#4668 CST, DE, 1:150), anti-total JNK (\#9255 CST, DE, 1:150), anti-FoxO1 (\#2880 CST, DE, 1:150), anti-LC3 (\#ab51520 Abcam, UK, 1:150), anti-GAPDH (\#8884 CST, DE, 1:1000). Secondary antibodies were anti-rabbit IgG HRP (CST, NE,\#7074)

Carbonylated proteins: To detect carbonylated proteins, snap frozen tissues were lysed in RIPA buffer and further processed using the OxyBlot Protein Oxidation Detection Kit (EMD Milipore, $\mathrm{GmbH}$ ) following the manufacturer's protocol. The carbonylated protein signal was normalized to total protein levels determined by Ponceau $S$ staining using densitometry measurements with ImageJ software. 
Immunoprecipitation: For immunoprecipitation experiments, mice were sacrificed by cervical dislocation after completion of the analysis. Muscles were surgically removed and snap frozen in liquid nitrogen. The tissues were homogenized in RIPA buffer containing protease and phosphatase inhibitors. Equal amount of protein, as determined by Bradford assay kit (Pierce, GmbH), was used for anti-HA immunoprecipitation following manufacturer recommendations (\#26180, Thermo Fisher Scientific, Gmbh). After immunoprecipitations, samples were subjected to SDS-PAGE, and further transferred to nitrocellulose membranes, incubated with primary anti-Pcyt2 antibody (HPA023033, Atlas Antibodies, Sweden) Followed by and a HRP-labelled secondary Anti-rabbit antibody (\#7074, CST, NE, \#7074).

ELISA: Tissue lysates in RIPA buffer containing protease and phosphatase inhibitors as above were used for ELISA measurements of IL-1 beta (\#RAB0275, Sigma Aldrich, GmbH) and IL-6 (\#RAB0309, Sigma Aldrich, GmbH) following manufacturer's recommendations. Absorbance values were measured using the Tecan microplate reader and data were processed using the Magellan software. Concentrations were determined based on standard curves and normalized to total protein concentrations of the corresponding sample determined by Bradford assay.

\section{RNA tissue extraction and Quantitative real-time PCR (RT-qPCR)}

Muscles or muscle biopsies were freshly isolated and flash frozen in liquid nitrogen. The mRNA was isolated using the RNeasy Lipid Tissue Mini Kit (Qiagen, GmbH). RNA concentration was estimated by measuring the absorbance at $260 \mathrm{~nm}$ using Nanodrop (Thermofisher, $\mathrm{GmbH}$ ). cDNA synthesis was performed using the iScript Advanced cDNA Synthesis Kit for RTqPCR (Bio-Rad, GmbH) following manufacturer's recommendations. cDNA was diluted in DNase-free water (1:10) before quantification by real-time PCR. mRNA transcript levels were measured in triplicate using the the CFX384 Real-Time PCR Detection System (BioRad) using the SybrGreen qPCR Master Mix (BioRad. GmBH). . Detection of the PCR products was attained with SYBR Green (Bio-Rad, GmbH). After completion of each run, melting curve analyses were performed to check for unspecific products, and representative samples run on agarose gels to ensure the specificity of amplification. Gene expression was normalized to the expression level of $18 \mathrm{~S}$ ribosomal rRNA as the reference gene. For normalization, 
threshold cycles (Ct-values) were normalized to 18 ribosomal rRNA within each sample to obtain sample-specific $\Delta \mathrm{Ct}$ values (= Ct gene of interest $-\mathrm{Ct}$ housekeeping gene). $2^{-\Delta \Delta C t}$ values were calculated to obtain fold expression levels. Primers sequences were as follows:

\section{SrRNA:}

FW 5'- GGCCGTTCTTAGTTGGTGGAGCG -3'

RV 5' - CTGAACGCCACTTGTCCCTC - 3'

Pcyt2:

FW 5'- CCGGTACAAGGGCAAGAACT -3'

RV 5' - TCTTCTCTTGGGCTCCTGGT - 3'

\section{Atrogin1:}

FW 5'- GCAAACACTGCCACATTCTCTC -3'

RV 5' - CTTGAGGGGAAAGTGAGACG - 3'

\section{MuRF1:}

FW 5' - ACCTGCTGGTGGAAAACATC -3'

RV 5'- ACCTGCTGGTGGAAAACATC - 3'

\section{Fbx031:}

FW 5'- GTATGGCGTTTGTGAGAACC -3'

RV 5' - AGCCCCAAAATGTGTCTGTA - 3'

\section{SrRNA (Human)}

FW 5'- GGCCCTGTAATTGGAATGAGTC -3'

RV 5'- CCAAGATCCAACTACGAGCTT - 3'

\section{PCYT2 (Human)}

FW 5'- GGTGCGATGGCTGCTATGA -3'

RV 5' - CACCTCGTCCACCCATTTGA - 3' 
Histology: Muscles were isolated from sacrificed animals and fixed in 4\%PFA for $72 \mathrm{hr}$. Tissues were embedded in paraffin blocks and $2 \mu \mathrm{m}$ sections were H\&E stained for histopathological examination. To address the effect of the 14-day eccentric exercise regime, animals were immediately sacrificed upon completion of the last exercise bout, muscles isolated and fixed in $4 \%$ PFA, and further processed for H\&E and toluidine blue staining. For determination of central nuclei quadriceps cross sections ( $n=4$ per group) were H\&E stained and 200-400 nuclei were counted. For extracellular collagen detection, $3 \mu \mathrm{m}$ paraffin muscle sections were stainded with Massone Trichrome staining kit according to manufacturer's recommendations (Abcam, UK, ab150686).

Immunocytochemistry: For immunocytochemistry, quadriceps muscles were harvested and fixed in PFA (4\%) for $72 \mathrm{~h}$ and cryoprotected by further immersing in $30 \%$ sucrose solution for another $72 \mathrm{hr}$. After embedding in OCT, sections were cut and stained using standard immunohistochemistry using the following antibodies: anti-Dystrophin (\#ab15277, Abcam, UK1:150), anti-Pax7 (P3U1, DSHB, lowa, US 1:150), antiDysferlin (\#ab124684, Abcam, UK); anti-CD3回? (\#ab49943, Abcam, UK 1:3000), anti-CD4 (\#14-9766-82, eBioscience, ThermoFisher, GmbH, 1:100), anti-CD8 (\#14-0808-82, ThermoFisher, GmbH, 1:100), Ly6G (\#127602, BioLegend, US, 1:500), anti-CIC3 (\#9661S, Cell Signaling, DE, 1:100), anti-B220 (\#550286, BD Biosciences, US, 1:50), and anti-F4/80 (\#MCA497G, BioRad, GmbH, 1:100).

Electron microcopy: For electron microcopy analysis, 6 month old animals were sacrificed, muscles were surgically excised, trimmed to small pieces and immediately immersed in cold $2.5 \%$ glutaraldehyde. Muscles were processed for electron microsopy as described previously 98. Briefly, tissue was cut into small pieces and fixed in $2.5 \%$ glutaraldehyde in $0.1 \mathrm{~mol} / \mathrm{l}$ sodium phosphate buffer, $\mathrm{pH}$ 7.4. for 1 hour. Subsequently samples were rinsed with the same buffer and post-fixed in $2 \%$ osmium tetroxide in $0.1 \mathrm{~mol} / \mathrm{l}$ sodium phosphate buffer on ice for $40 \mathrm{~min}$. After 3 rinsing steps in $\mathrm{ddH} 2 \mathrm{O}$, the samples were dehydrated in a graded series of acetone on ice and embedded in Agar 100 resin. 70-nm sections were post-stained with $2 \%$ uranyl acetate and Reynolds lead citrate. Sections were examined with a FEI Morgagni 268D (FEl, Eindhoven, The Netherlands) operated at $80 \mathrm{kV}$. Images were acquired using an 11 megapixel Morada CCD camera (Olympus-SIS). 


\section{In vivo muscle permeability measurements}

To determine in vivo muscle tissue permeability, we used the well described Evans blue dye assay ${ }^{57}$ Briefly, $1 \%$ Evans blue dye $\left(10 \mathrm{ml} \mathrm{kg}^{-1}\right.$ body weight, Sigma, $\left.\mathrm{GmbH}\right)$ was injected into the intraperitoneal cavity of 8 month old animals. 16hours later mice were sacrificed via cervical dislocation and muscles collected. Quadriceps muscles were weighed and then soaked in formamide (GibcoBRL, UK) for $48 \mathrm{hr}$ at $55^{\circ} \mathrm{C}$ with gentle shaking. The optical density of Evans blue in the resulting supernatant was measured at $610 \mathrm{~nm}$ with a Spectronic 610 spectrophotometer (Milton Roy). To image Evans blue via microscopy, muscles were immediately embedded in OCT and frozen. 10? $\mathrm{m}$ longitudinal sections were cut and visualized after counterstaining with DAPI.

\section{Pcyt2 enzyme activity}

Pcyt2 activity was measured as previously described ${ }^{17}$. Briefly, frozen muscle tissue (50mg) was homogenized in a cold lysis buffer (10 mM Tris- $\mathrm{HCl}$ [pH 7.4], 1 mM EDTA, and $10 \mathrm{mM}$ $\mathrm{NaF}$ ) and briefly centrifuged to remove cell debris. Fifty ug of protein was assayed with 0.2 $\mu \mathrm{Ci}$ of [14C]-phosphoethanolamine (P-Etn) (American Radiolabeled Chemical) in $50 \mu \mathrm{l}$ of reaction mixture of $50 \mathrm{mM} \mathrm{MgCl} 2,50 \mathrm{mM}$ DTT, $10 \mathrm{mM}$ unlabeled P-Etn, $20 \mathrm{mM}$ CTP and 100 $\mathrm{mM}$ Tris- $\mathrm{HCl}\left(\mathrm{pH} \mathrm{7.8)}\right.$. The reaction was incubated at $37^{\circ} \mathrm{C}$ for $15 \mathrm{~min}$ and terminated by boiling (2 min). The reaction product [14C]CDP-Etn was separated from [14-C]PEtn on Silica gel G plates (Analtech) with a solvent system of methanol:0.5\% NaCl:ammonia (10:10:1). CDP- Etn and P-Etn in standards and samples were visualized with a $0.5 \%$ ninhydrin in ethanol and the [14C]CDP-Etn collected and quantified by liquid-scintillation counting. Pcyt2 activity was expressed in $\mathrm{nmol} / \mathrm{min} / \mathrm{mg}$ protein. Protein content was determined with bicinchronic acid assay from Pierce.

\section{Statistical analysis of mouse studies}

All mouse data are expressed as mean +/- standard error of the mean (SEM). Statistical significance was tested by Student's two tailed, unpaired t-test; Mann-Whitney U test; 2-way ANOVA followed by Bonferroni's post-hoc test or Analysis of Co-Variance (ANCOVA) ${ }^{86}$. All 
bioRxiv preprint doi: https://doi.org/10.1101/2022.03.02.482658; this version posted March 3, 2022. The copyright holder for this preprint (which was not certified by peer review) is the author/funder. All rights reserved. No reuse allowed without permission.

figures and mouse statistical analyses were generated using Prism 8 (GraphPad) or R. Details of the statistical tests used are stated in the figure legends. In all figures, statistical significance is represented as $* P<0.05, * * P<0.01, * * * P<0.001, * * * * P<0.0001$. 


\section{References}

1. Davies, K. E. \& Nowak, K. J. Molecular mechanisms of muscular dystrophies: Old and new players. Nature Reviews Molecular Cell Biology (2006) doi:10.1038/nrm2024.

2. Wilkinson, D. J., Piasecki, M. \& Atherton, P. J. The age-related loss of skeletal muscle mass and function: Measurement and physiology of muscle fibre atrophy and muscle fibre loss in humans. Ageing Research Reviews (2018) doi:10.1016/j.arr.2018.07.005.

3. Shevchenko, A. \& Simons, K. Lipidomics: Coming to grips with lipid diversity. Nature Reviews Molecular Cell Biology (2010) doi:10.1038/nrm2934.

4. Harayama, T. \& Riezman, H. Understanding the diversity of membrane lipid composition. Nature Reviews Molecular Cell Biology (2018) doi:10.1038/nrm.2017.138.

5. Alessenko, A. V. \& Albi, E. Exploring Sphingolipid Implications in Neurodegeneration. Frontiers in Neurology (2020) doi:10.3389/fneur.2020.00437.

6. Gibellini, F. \& Smith, T. K. The Kennedy pathway-de novo synthesis of phosphatidylethanolamine and phosphatidylcholine. IUBMB Life (2010) doi:10.1002/iub.337.

7. Vaz, F. M. et al. Mutations in PCYT2 disrupt etherlipid biosynthesis and cause a complex hereditary spastic paraplegia. Brain (2019) doi:10.1093/brain/awz291.

8. Ahmed, M. Y. et al. A mutation of EPT1 (SELENOI) underlies a new disorder of Kennedy pathway phospholipid biosynthesis. Brain (2017) doi:10.1093/brain/aww318.

9. Horibata, Y. et al. EPT1 (selenoprotein I) is critical for the neural development and maintenance of plasmalogen in humans. J. Lipid Res. (2018) doi:10.1194/jlr.P081620.

10. Horibata, Y. \& Hirabayashi, Y. Identification and characterization of human ethanolaminephosphotransferase. J. Lipid Res. (2007) doi:10.1194/jlr.C600019- 
JLR200.

11. Uhlén, M. et al. Tissue-based map of the human proteome. Science (80-. ). (2015) doi:10.1126/science.1260419.

12. Peres, J. N. \& Houart, C. Modeling Hereditary Spastic Paraplegia (HSP) in Zebrafish. in Movement Disorders: Genetics and Models: Second Edition (2015). doi:10.1016/B9780-12-405195-9.00072-X.

13. Naef, V. et al. Swimming in Deep Water: Zebrafish Modeling of Complicated Forms of Hereditary Spastic Paraplegia and Spastic Ataxia. Frontiers in Neuroscience (2019) doi:10.3389/fnins.2019.01311.

14. Fassier, C., Hazan, J. \& Melki, J. Mouse Models of Autosomal Dominant Spastic Paraplegia. in Movement Disorders: Genetics and Models: Second Edition (2015). doi:10.1016/B978-0-12-405195-9.00070-6.

15. Blackstone, C. Murine Models of Autosomal Recessive Hereditary Spastic Paraplegia. in Movement Disorders: Genetics and Models: Second Edition (2015). doi:10.1016/B978-0-12-405195-9.00071-8.

16. Rapisarda, R., Muntoni, F., Gobbi, P. \& Dubowitz, V. Duchenne muscular dystrophy presenting with failure to thrive. Arch. Dis. Child. (1995) doi:10.1136/adc.72.5.437.

17. Fullerton, M. D., Hakimuddin, F. \& Bakovic, M. Developmental and Metabolic Effects of Disruption of the Mouse CTP:Phosphoethanolamine Cytidylyltransferase Gene (Pcyt2). Mol. Cell. Biol. (2007) doi:10.1128/mcb.01527-06.

18. Francetic, T. \& Li, Q. Skeletal myogenesis and Myf5 activation. Transcription (2011) doi:10.4161/trns.2.3.15829.

19. White, R. B., Biérinx, A. S., Gnocchi, V. F. \& Zammit, P. S. Dynamics of muscle fibre growth during postnatal mouse development. BMC Dev. Biol. (2010) doi:10.1186/1471-213X-10-21.

20. Mccarthy, J. J. et al. Effective fiber hypertrophy in satellite cell-depleted skeletal muscle. Development (2011) doi:10.1242/dev.068858. 
21. Archer, J. E., Gardner, A. C., Roper, H. P., Chikermane, A. A. \& Tatman, A. J. Duchenne muscular dystrophy: the management of scoliosis. J. Spine Surg. (2016) doi:10.21037/jss.2016.08.05.

22. McGreevy, J. W., Hakim, C. H., McIntosh, M. A. \& Duan, D. Animal models of Duchenne muscular dystrophy: From basic mechanisms to gene therapy. DMM Disease Models and Mechanisms (2015) doi:10.1242/dmm.018424.

23. Seale, P. et al. PRDM16 controls a brown fat/skeletal muscle switch. Nature (2008) doi:10.1038/nature07182.

24. Eguchi, J. et al. Transcriptional control of adipose lipid handling by IRF4. Cell Metab. (2011) doi:10.1016/j.cmet.2011.02.005.

25. Weiland, D. et al. Imbalance of Mitochondrial Respiratory Chain Complexes in the Epidermis Induces Severe Skin Inflammation. J. Invest. Dermatol. (2018) doi:10.1016/j.jid.2017.08.019.

26. Shin, J. M. et al. Targeted deletion of Crif1 in mouse epidermis impairs skin homeostasis and hair morphogenesis. Sci. Rep. (2017) doi:10.1038/srep44828.

27. Nguyen, T. T. et al. Loss of Miro1-directed mitochondrial movement results in a novel murine model for neuron disease. Proc. Natl. Acad. Sci. U. S. A. (2014) doi:10.1073/pnas.1402449111.

28. Choi, M. J. et al. An adipocyte-specific defect in oxidative phosphorylation increases systemic energy expenditure and protects against diet-induced obesity in mouse models. Diabetologia (2020) doi:10.1007/s00125-019-05082-7.

29. Vernochet, C. et al. Adipose tissue mitochondrial dysfunction triggers a lipodystrophic syndrome with insulin resistance, hepatosteatosis, and cardiovascular complications. FASEB J. (2014) doi:10.1096/fj.14-253971.

30. Sustarsic, E. G. et al. Cardiolipin Synthesis in Brown and Beige Fat Mitochondria Is Essential for Systemic Energy Homeostasis. Cell Metab. (2018) doi:10.1016/j.cmet.2018.05.003. 
31. Srivillibhuthur, M. et al. TFAM is required for maturation of the fetal and adult intestinal epithelium. Dev. Biol. (2018) doi:10.1016/j.ydbio.2018.04.015.

32. Brüning, J. C. et al. A muscle-specific insulin receptor knockout exhibits features of the metabolic syndrome of NIDDM without altering glucose tolerance. Mol. Cell (1998) doi:10.1016/S1097-2765(00)80155-0.

33. Chamberlain, J. S., Jaynes, J. B. \& Hauschka, S. D. Regulation of creatine kinase induction in differentiating mouse myoblasts. Mol. Cell. Biol. (1985) doi:10.1128/mcb.5.3.484.

34. Selathurai, A. et al. The CDP-Ethanolamine Pathway Regulates Skeletal Muscle Diacylglycerol Content and Mitochondrial Biogenesis without Altering Insulin Sensitivity. Cell Metab. (2015) doi:10.1016/j.cmet.2015.04.001.

35. Beedle, A. M. et al. Mouse fukutin deletion impairs dystroglycan processing and recapitulates muscular dystrophy. J. Clin. Invest. (2012) doi:10.1172/JCI63004.

36. Potthoff, M. J. et al. Regulation of Skeletal Muscle Sarcomere Integrity and Postnatal Muscle Function by Mef2c. Mol. Cell. Biol. (2007) doi:10.1128/mcb.01187-07.

37. Schlame, M., Xu, Y., Erdjument-Bromage, H., Neubert, T. A. \& Ren, M. Lipidome-wide 13C flux analysis: A novel tool to estimate the turnover of lipids in organisms and cultures. J. Lipid Res. (2020) doi:10.1194/jlr.D119000318.

38. Nair, U. et al. A role for Atg8-PE deconjugation in autophagosome biogenesis. Autophagy (2012) doi:10.4161/auto.19385.

39. Martinez-Lopez, N. et al. Autophagy in Myf5+ progenitors regulates energy and glucose homeostasis through control of brown fat and skeletal muscle development. EMBO Rep. (2013) doi:10.1038/embor.2013.111.

40. Chimienti, G. et al. Deletion of ogg1 results in a differential signature of oxidized purine base damage in mtdna regions. Int. J. Mol. Sci. (2019) doi:10.3390/ijms20133302.

41. DiMauro, S. Mitochondrial diseases. Biochimica et Biophysica Acta-Bioenergetics 
(2004) doi:10.1016/j.bbabio.2004.03.014.

42. Hansson, A. et al. A switch in metabolism precedes increased mitochondrial biogenesis in respiratory chain-deficient mouse hearts. Proc. Natl. Acad. Sci. U. S. A. (2004) doi:10.1073/pnas.0308710100.

43. Luft, R., Ikkos, D., Palmieri, G., Ernster, L. \& Afzelius, B. A CASE OF SEVERE HYPERMETABOLISM OF NONTHYROID ORIGIN WITH A DEFECT IN THE MAINTENANCE OF MITOCHONDRIAL RESPIRATORY CONTROL: A CORRELATED CLINICAL, BIOCHEMICAL, AND MORPHOLOGICAL STUDY. J. Clin. Invest. (1962) doi:10.1172/jci104637.

44. Shy, G. M. \& Gonatas, N. K. Human myopathy with giant abnormal mitochondria. Science (80-. ). (1964) doi:10.1126/science.145.3631.493.

45. SHY, G. M., GONATAS, N. K. \& PEREZ, M. TWO CHILDHOOD MYOPATHIES WITH ABNORMAL MITOCHONDRIA. Brain (1966) doi:10.1093/brain/89.1.133.

46. Uittenbogaard, M. \& Chiaramello, A. Mitochondrial Biogenesis: A Therapeutic Target for Neurodevelopmental Disorders and Neurodegenerative Diseases. Curr. Pharm. Des. (2014) doi:10.2174/1381612820666140305224906.

47. Joza, N. et al. Muscle-Specific Loss of Apoptosis-Inducing Factor Leads to Mitochondrial Dysfunction, Skeletal Muscle Atrophy, and Dilated Cardiomyopathy. Mol. Cell. Biol. (2005) doi:10.1128/mcb.25.23.10261-10272.2005.

48. Tezze, C. et al. Age-Associated Loss of OPA1 in Muscle Impacts Muscle Mass, Metabolic Homeostasis, Systemic Inflammation, and Epithelial Senescence. Cell Metab. (2017) doi:10.1016/j.cmet.2017.04.021.

49. Ishihara, T. et al. Dynamics of Mitochondrial DNA Nucleoids Regulated by Mitochondrial Fission Is Essential for Maintenance of Homogeneously Active Mitochondria during Neonatal Heart Development. Mol. Cell. Biol. (2015) doi:10.1128/mcb.01054-14.

50. Puccio, H. et al. Mouse models for Friedreich ataxia exhibit cardiomyopathy, sensory 
nerve defect and Fe-S enzyme deficiency followed by intramitochondrial iron deposits. Nat. Genet. (2001) doi:10.1038/84818.

51. Selathurai, A. et al. Phosphatidylserine decarboxylase is critical for the maintenance of skeletal muscle mitochondrial integrity and muscle mass. Mol. Metab. (2019) doi:10.1016/j.molmet.2019.06.020.

52. Van Meer, G., Voelker, D. R. \& Feigenson, G. W. Membrane lipids: Where they are and how they behave. Nature Reviews Molecular Cell Biology (2008) doi:10.1038/nrm2330.

53. Roses, A. D. \& Appel, S. H. Inherited Membrane Disorders of Muscle: Duchenne Muscular Dystrophy and Myotonic Muscular Dystrophy. in Physiology of Membrane Disorders (1978). doi:10.1007/978-1-4613-3958-8_38.

54. Steinkühler, J., Sezgin, E., Urbančič, I., Eggeling, C. \& Dimova, R. Mechanical properties of plasma membrane vesicles correlate with lipid order, viscosity and cell density. Commun. Biol. (2019) doi:10.1038/s42003-019-0583-3.

55. Elsayad, K. et al. Mapping the subcellular mechanical properties of live cells in tissues with fluorescence emission-Brillouin imaging. Sci. Signal. (2016) doi:10.1126/scisignal.aaf6326.

56. Demonbreun, A. R. \& McNally, E. M. Plasma Membrane Repair in Health and Disease. Curr. Top. Membr. (2016) doi:10.1016/bs.ctm.2015.10.006.

57. Hamer, P. W., McGeachie, J. M., Davies, M. J. \& Grounds, M. D. Evans Blue Dye as an in vivo marker of myofibre damage: Optimising parameters for detecting initial myofibre membrane permeability. J. Anat. (2002) doi:10.1046/j.00218782.2001.00008.x.

58. Bansal, D. et al. Defective membrane repair in dysferlin-deficient muscular dystrophy. Nature (2003) doi:10.1038/nature01573.

59. Hody, S., Croisier, J. L., Bury, T., Rogister, B. \& Leprince, P. Eccentric muscle contractions: Risks and benefits. Frontiers in Physiology (2019) 
doi:10.3389/fphys.2019.00536.

60. Bulaklak, K. \& Gersbach, C. A. The once and future gene therapy. Nature Communications (2020) doi:10.1038/s41467-020-19505-2.

61. Dunbar, C. E. et al. Gene therapy comes of age. Science (2018) doi:10.1126/science.aan4672.

62. Ramos, J. N. et al. Development of Novel Micro-dystrophins with Enhanced Functionality. Mol. Ther. (2019) doi:10.1016/j.ymthe.2019.01.002.

63. Crudele, J. M. \& Chamberlain, J. S. AAV-based gene therapies for the muscular dystrophies. Human Molecular Genetics (2019) doi:10.1093/hmg/ddz128.

64. Rice, K. M., Preston, D. L., Neff, D., Norton, M. \& Blough, E. R. Age-related dystrophinglycoprotein complex structure and function in the rat extensor digitorum longus and soleus muscle. Journals Gerontol. - Ser. A Biol. Sci. Med. Sci. (2006) doi:10.1093/gerona/61.11.1119.

65. Hord, J. M., Botchlett, R. \& Lawler, J. M. Age-related alterations in the sarcolemmal environment are attenuated by lifelong caloric restriction and voluntary exercise. Exp. Gerontol. (2016) doi:10.1016/j.exger.2016.08.006.

66. Ramaswamy, K. S. et al. Lateral transmission of force is impaired in skeletal muscles of dystrophic mice and very old rats. J. Physiol. (2011) doi:10.1113/jphysiol.2010.201921.

67. Lovering, R. M. \& Brooks, S. V. Eccentric exercise in aging and diseased skeletal muscle: Good or bad? Journal of Applied Physiology (2014) doi:10.1152/japplphysiol.00174.2013.

68. Micewicz, E. D. et al. Small lipidated anti-obesity compounds derived from neuromedin U. Eur. J. Med. Chem. (2015) doi:10.1016/j.ejmech.2015.07.020.

69. Gault, M. L. \& Willems, M. E. T. Aging, functional capacity and eccentric exercise training. Aging and Disease (2013) doi:10.14336/AD.2013.0400351.

70. Manfredi, T. G. et al. Plasma creatine kinase activity and exercise-induced muscle 
damage in older men. Med. Sci. Sports Exerc. (1991) doi:10.1249/00005768$199109000-00006$.

71. Roth, S. M. et al. High-volume, heavy-resistance strength training and muscle damage in young and older women. J. Appl. Physiol. (2000) doi:10.1152/jappl.2000.88.3.1112.

72. Möller, M. N., Lancaster, J. R. \& Denicola, A. Chapter 2 The Interaction of Reactive Oxygen and Nitrogen Species with Membranes. Current Topics in Membranes (2008) doi:10.1016/S1063-5823(08)00202-0.

73. Yusupov, M. et al. Effect of head group and lipid tail oxidation in the cell membrane revealed through integrated simulations and experiments. Sci. Rep. (2017) doi:10.1038/s41598-017-06412-8.

74. Meyers, R. M. et al. Computational correction of copy number effect improves specificity of CRISPR-Cas9 essentiality screens in cancer cells. Nat. Genet. (2017) doi:10.1038/ng.3984.

75. Dawaliby, R. et al. Phosphatidylethanolamine is a key regulator of membrane fluidity in eukaryotic cells. J. Biol. Chem. (2016) doi:10.1074/jbc.M115.706523.

76. Shavlakadze, T. et al. Age-Related Gene Expression Signature in Rats Demonstrate Early, Late, and Linear Transcriptional Changes from Multiple Tissues. Cell Rep. (2019) doi:10.1016/j.celrep.2019.08.043.

77. Cree, M. G. et al. Intramuscular and liver triglycerides are increased in the elderly. J. Clin. Endocrinol. Metab. (2004) doi:10.1210/jc.2003-031986.

78. Pavlovic, Z. \& Bakovic, M. Regulation of phosphatidylethanolamine homeostasis-the critical role of CTP: Phosphoethanolamine cytidylyltransferase (Pcyt2). International Journal of Molecular Sciences (2013) doi:10.3390/ijms14022529.

79. Bergström, J. \& Hultman, E. A study of the glycogen metabolism during exercise in man. Scand. J. Clin. Lab. Invest. (1967) doi:10.3109/00365516709090629.

80. Leonardi, R., Frank, M. W., Jackson, P. D., Rock, C. O. \& Jackowski, S. Elimination of the CDP-ethanolamine pathway disrupts hepatic lipid homeostasis. J. Biol. Chem. 
(2009) doi:10.1074/jbc.M109.031336.

81. Guyenet, S. J. et al. A simple composite phenotype scoring system for evaluating mouse models of cerebellar ataxia. J. Vis. Exp. (2010) doi:10.3791/1787.

82. Cai, C. et al. MG53 nucleates assembly of cell membrane repair machinery. Nat. Cell Biol. (2009) doi:10.1038/ncb1812.

83. Koltai, E. et al. SIRT1 may play a crucial role in overload-induced hypertrophy of skeletal muscle. J. Physiol. (2017) doi:10.1113/JP273774.

84. Halbert, C. L., Allen, J. M. \& Chamberlain, J. S. AAV6 vector production and purification for muscle gene therapy. in Methods in Molecular Biology (2018). doi:10.1007/978-1-4939-7374-3_18.

85. Orthofer, M. et al. Identification of ALK in Thinness. Cell (2020) doi:10.1016/j.cell.2020.04.034.

86. Tschöp, M. H. et al. A guide to analysis of mouse energy metabolism. Nature Methods (2012) doi:10.1038/nmeth.1806.

87. Shahini, A. et al. Efficient and high yield isolation of myoblasts from skeletal muscle. Stem Cell Res. (2018) doi:10.1016/j.scr.2018.05.017.

88. Fasshauer, M. et al. Essential Role of Insulin Receptor Substrate 1 in Differentiation of Brown Adipocytes. Mol. Cell. Biol. (2001) doi:10.1128/mcb.21.1.319-329.2001.

89. Köberlin, M. S. et al. A Conserved Circular Network of Coregulated Lipids Modulates Innate Immune Responses. Cell (2015) doi:10.1016/j.cell.2015.05.051.

90. Sezgin, E. et al. Elucidating membrane structure and protein behavior using giant plasma membrane vesicles. Nat. Protoc. (2012) doi:10.1038/nprot.2012.059.

91. Sezgin, E., Waithe, D., Bernardino De La Serna, J. \& Eggeling, C. Spectral imaging to measure heterogeneity in membrane lipid packing. ChemPhysChem (2015) doi:10.1002/cphc.201402794.

92. Elsayad, K. et al. Mechanical Properties of cellulose fibers measured by Brillouin 
spectroscopy. Cellulose (2020) doi:10.1007/s10570-020-03075-z.

93. Scarcelli, G. et al. Noncontact three-dimensional mapping of intracellular hydromechanical properties by Brillouin microscopy. Nat. Methods (2015) doi:10.1038/nmeth.3616.

94. Edrei, E., Gather, M. C. \& Scarcelli, G. Integration of spectral coronagraphy within VIPA-based spectrometers for high extinction Brillouin imaging. Opt. Express (2017) doi:10.1364/oe.25.006895.

95. Meng, Z., Traverso, A. J. \& Yakovlev, V. V. Background clean-up in Brillouin microspectroscopy of scattering medium. Opt. Express (2014) doi:10.1364/oe.22.005410.

96. Xiao, S., Weiner, A. M. \& Lin, C. A dispersion law for virtually imaged phased-array spectral dispersers based on paraxial wave theory. IEEE J. Quantum Electron. (2004) doi:10.1109/JQE.2004.825210.

97. Elsayad, K. Spectral phasor analysis for brillouin microspectroscopy. Front. Phys. (2019) doi:10.3389/fphy.2019.00062.

98. Takeshima, H., Komazaki, S., Nishi, M., lino, M. \& Kangawa, K. Junctophilins: A novel family of junctional membrane complex proteins. Mol. Cell (2000) doi:10.1016/s10972765(05)00005-5. 


\section{Figure 1}

A

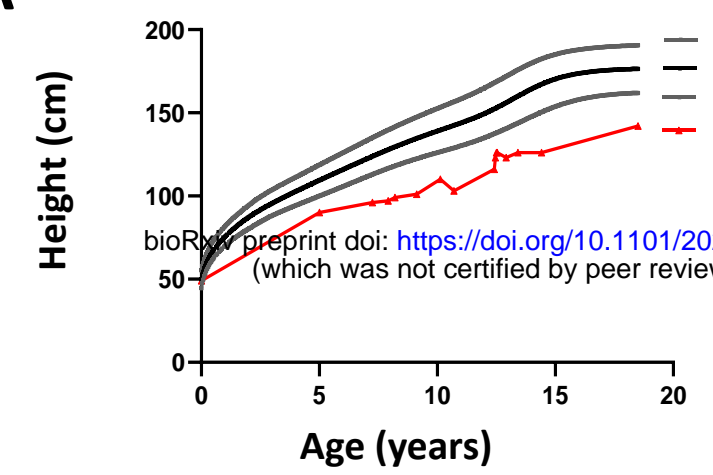

C

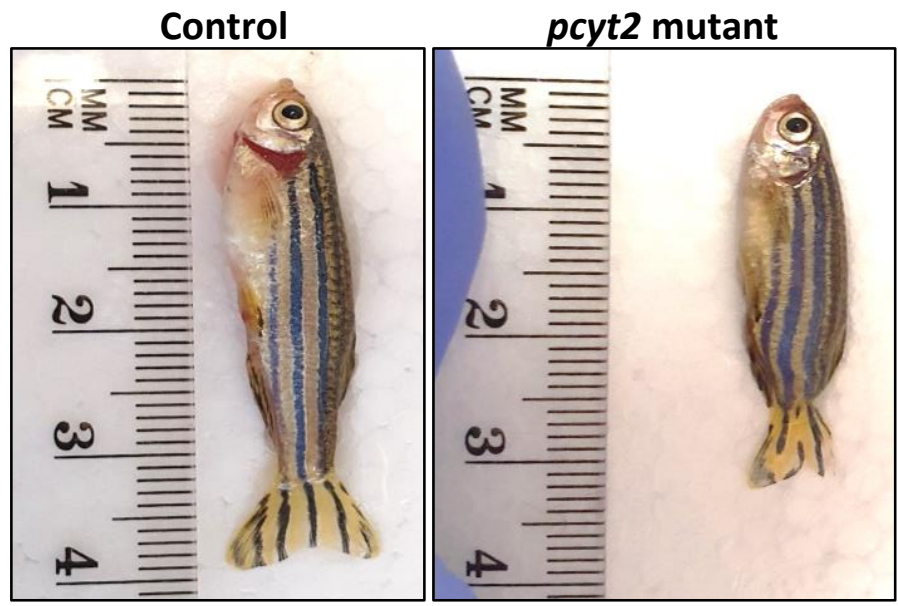

D
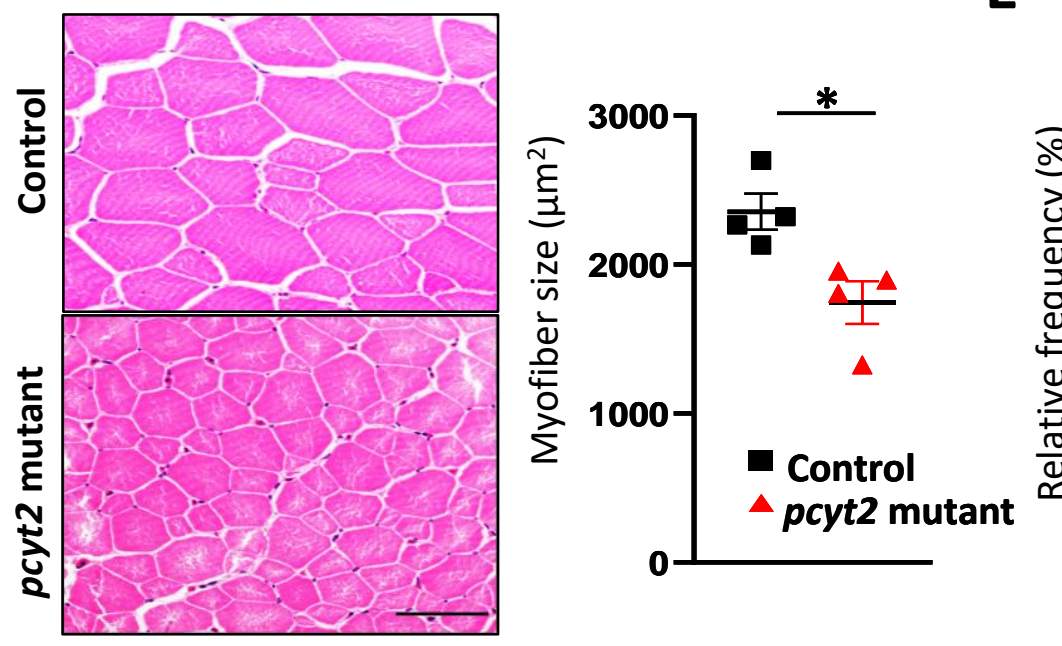

$\mathbf{G}$

$\mathbf{F}$

$$
\begin{aligned}
& \text { F }
\end{aligned}
$$

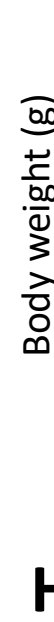

Control

Myf5Cre-Pcyt2

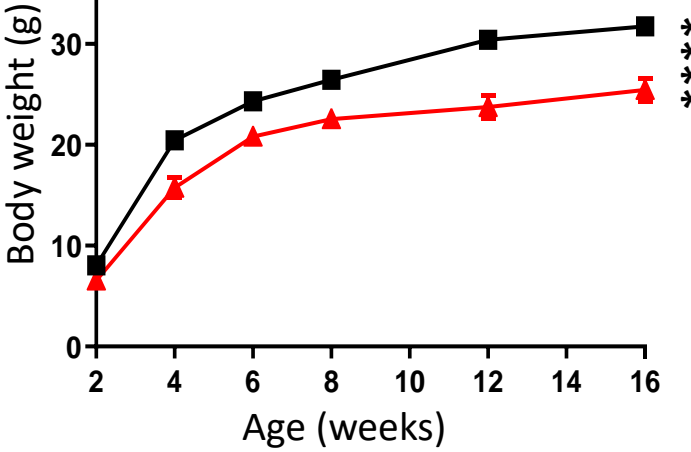

H

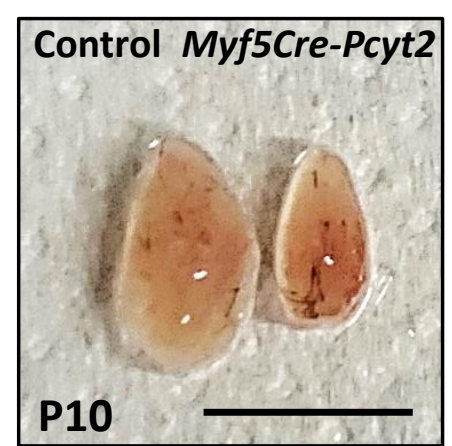

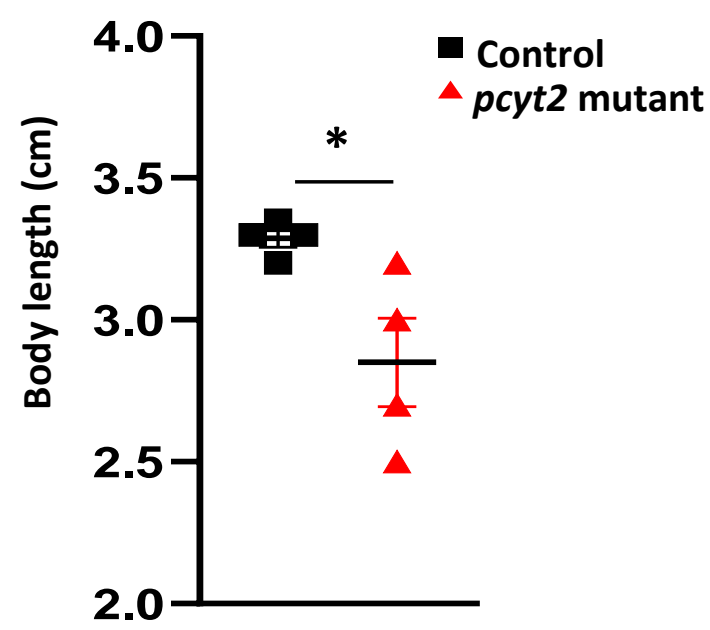

E

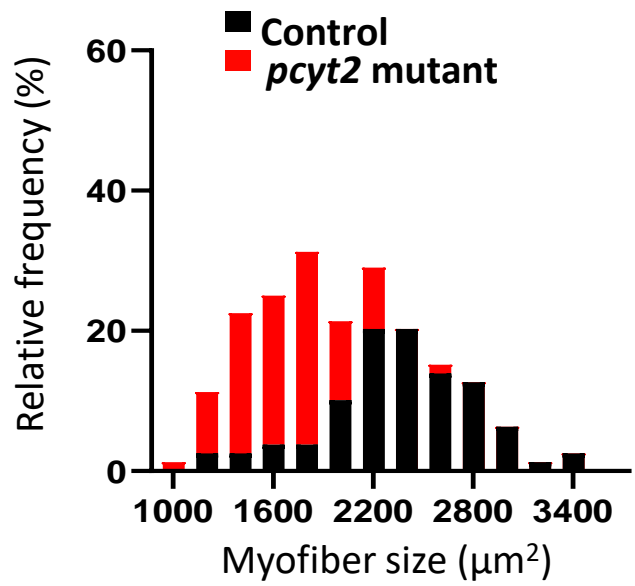

B
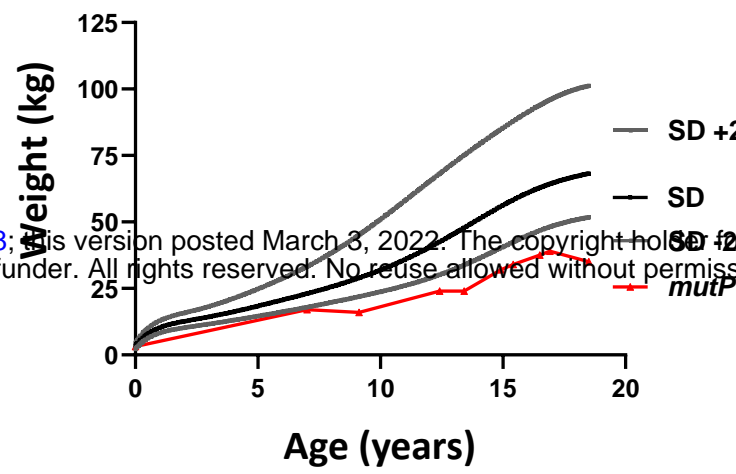

Myofiber size $\left(\mu \mathrm{m}^{2}\right)$
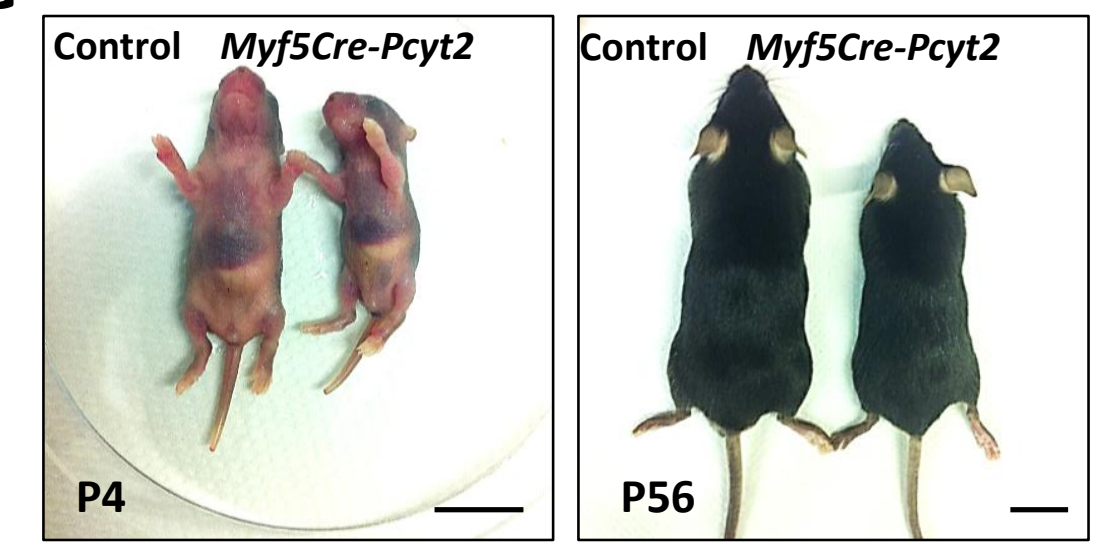

J

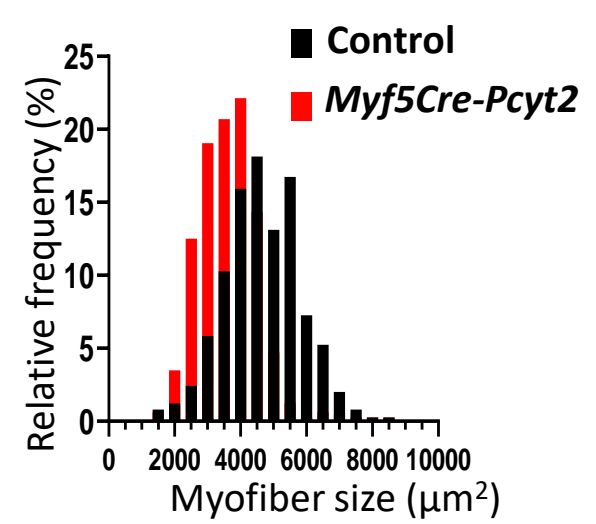


Figure 2

A Control

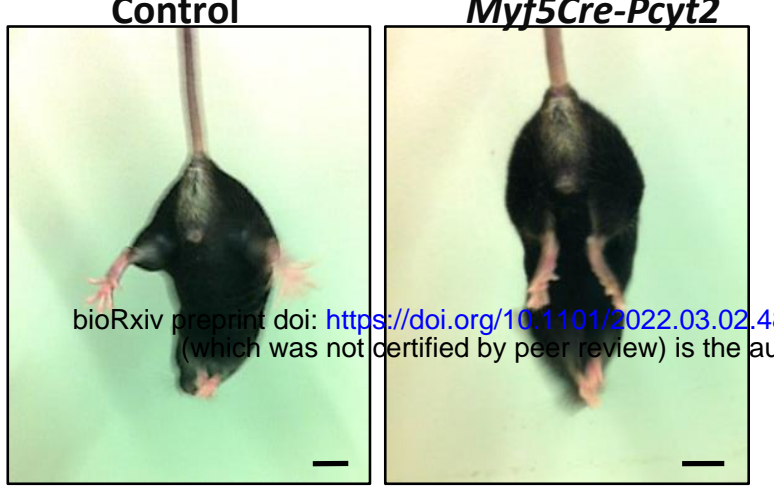

C

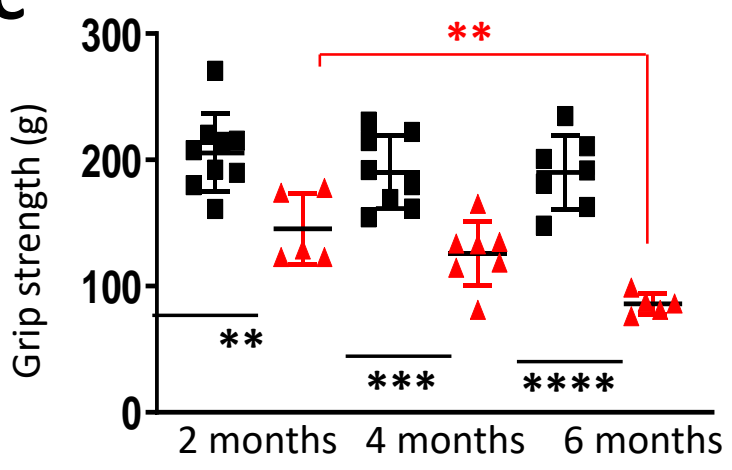

D
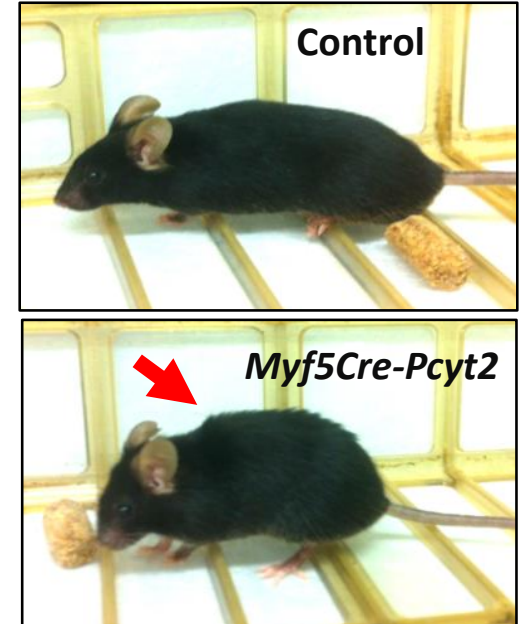

F

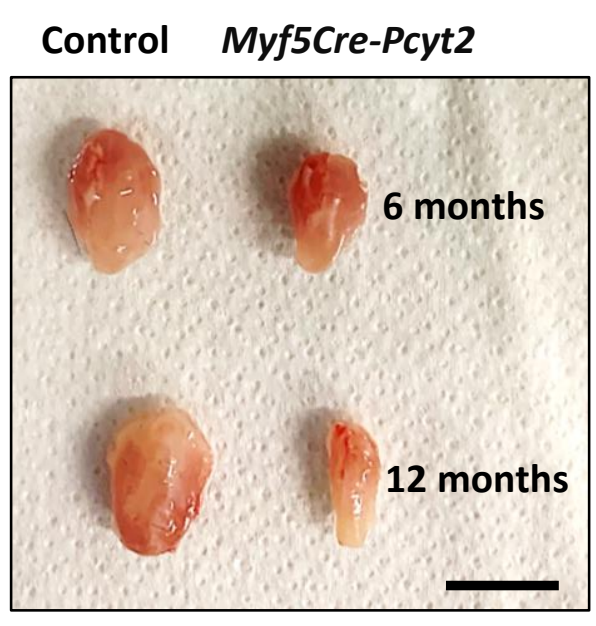

H

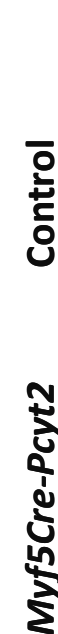

Control

Myf5Cre-Pcyt2
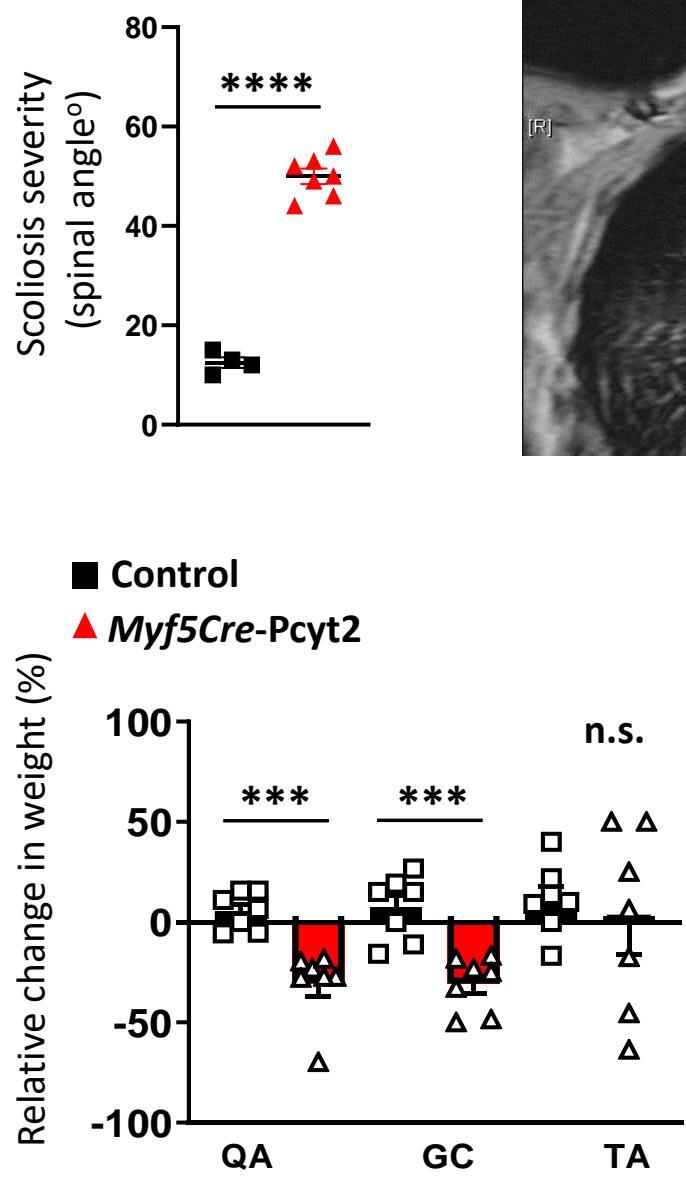

G

Control

A Myf5Cre-Pcyt2

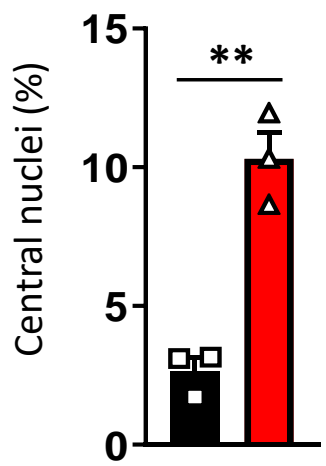

J

- Control

- Myf5Cre-Pcyt2
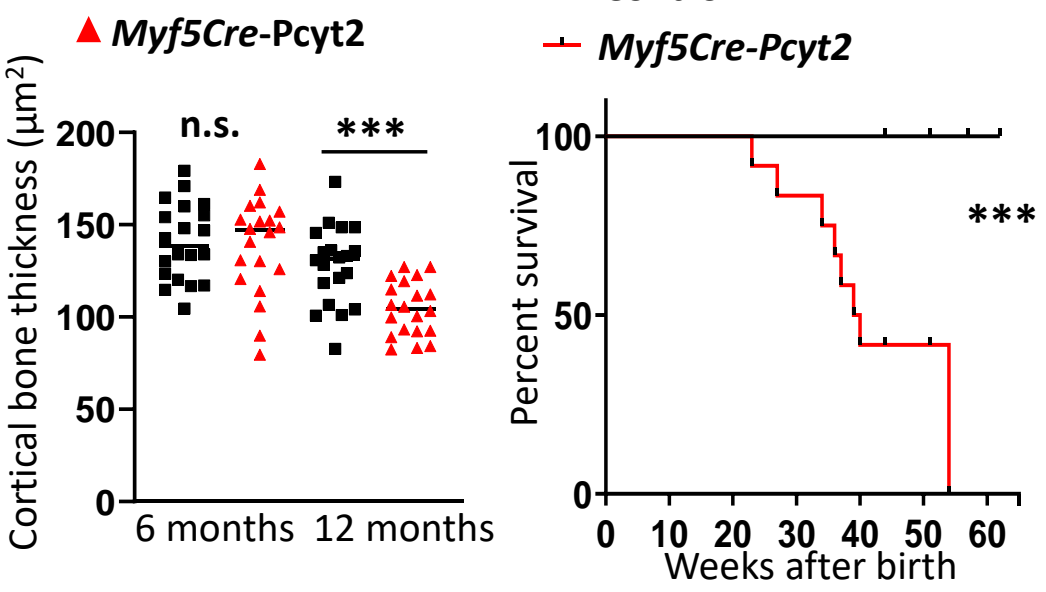
A

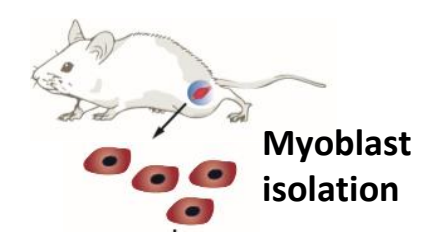

i.

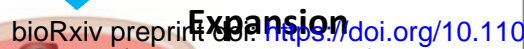
- (which was not certified by peer

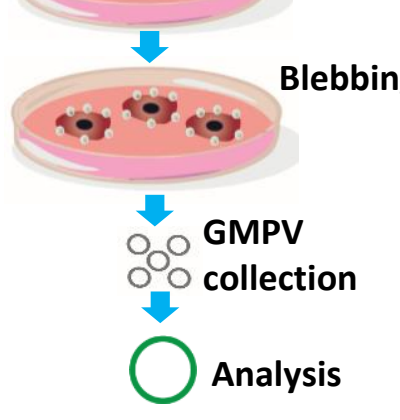

C

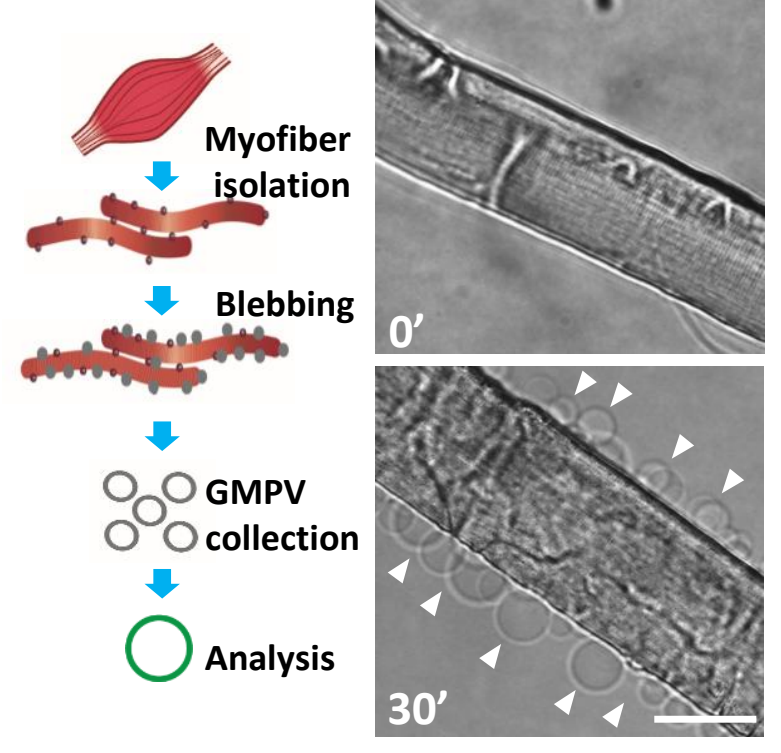

Membrane Packing and Rigidity (GP)

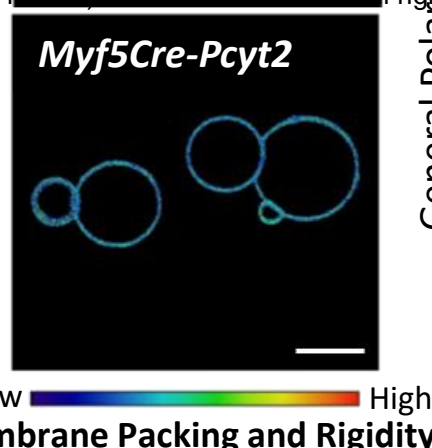

D

$\mathbf{E}$

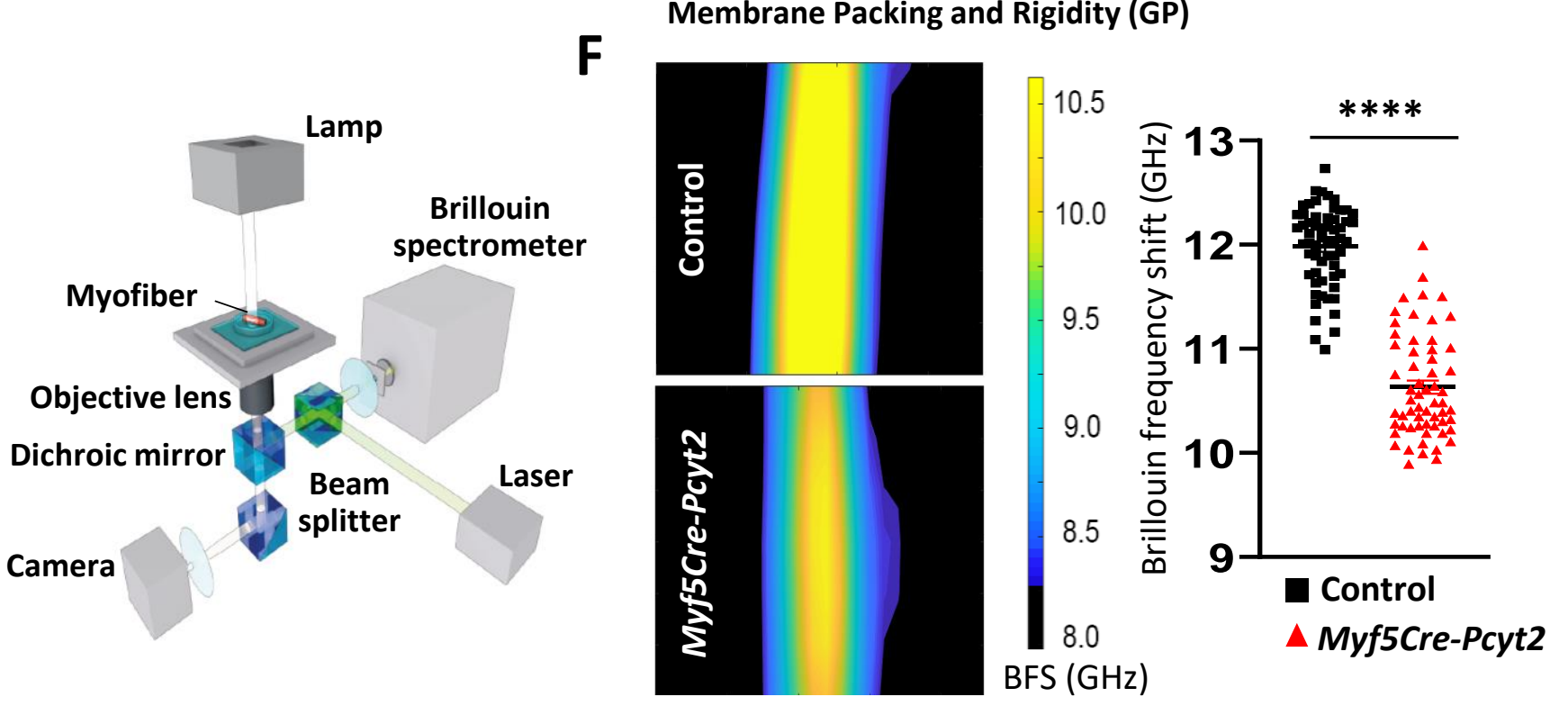

G

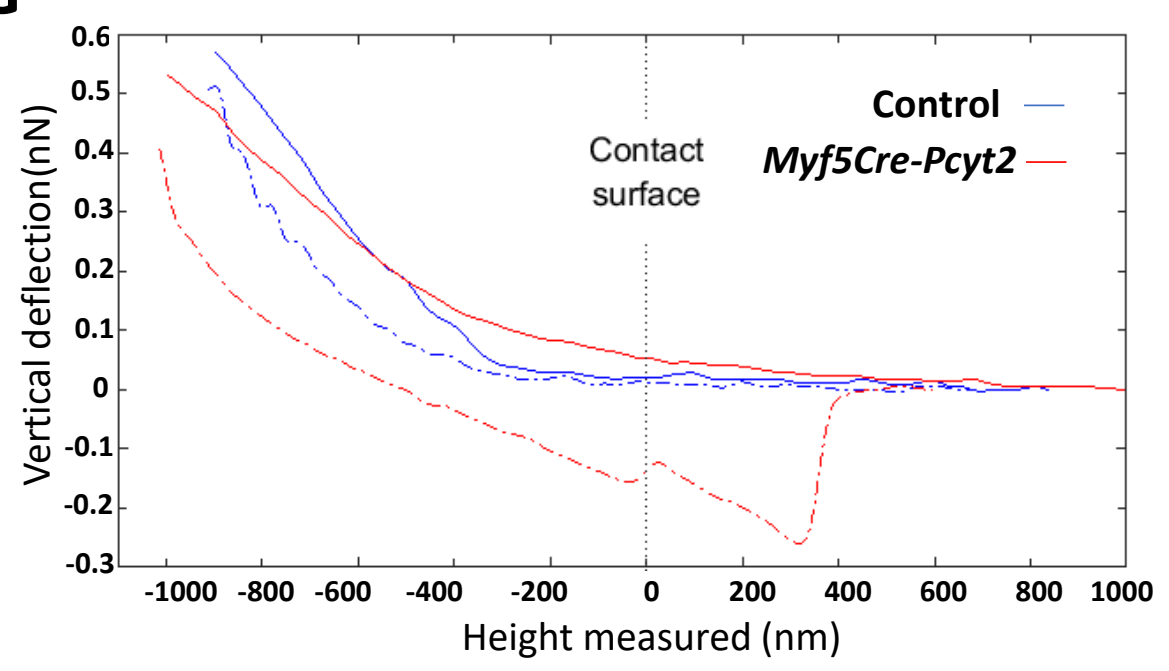

H Control

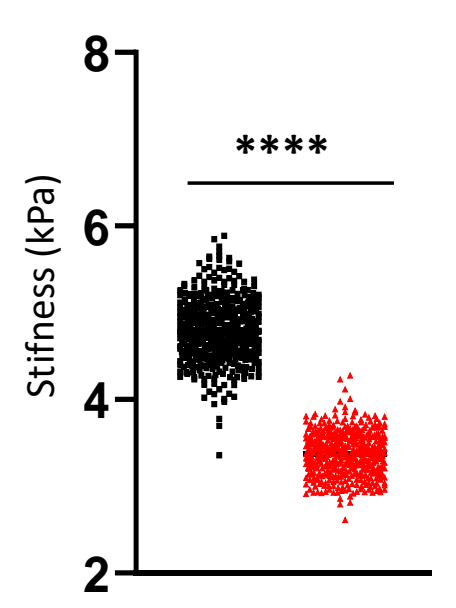
His reserved. No rume-nowed withoyt permission.

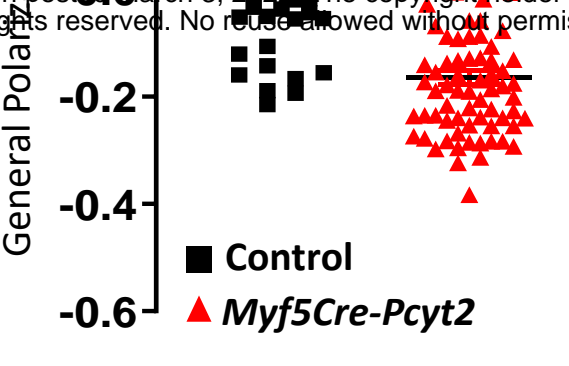

\section{Control}
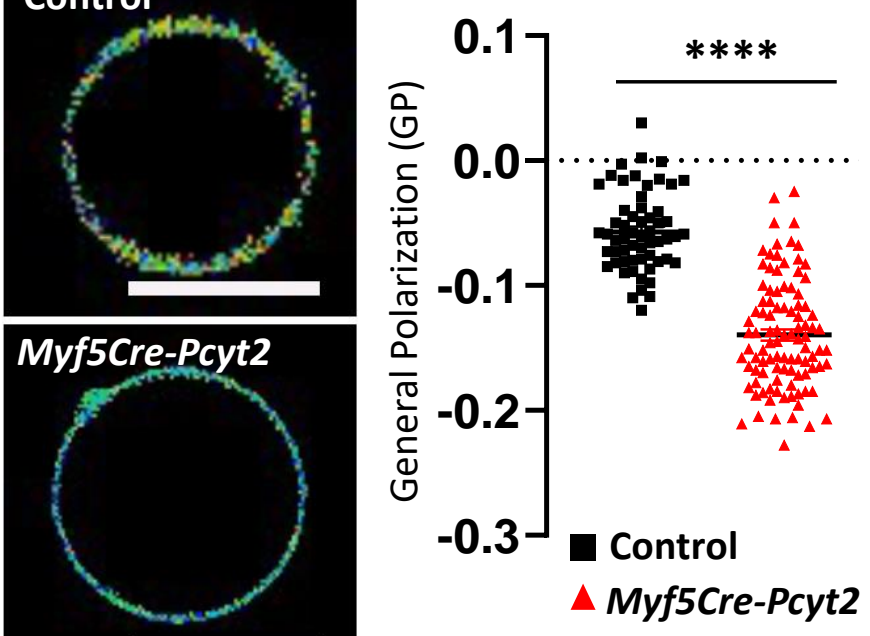

Low High

Membrane Packing and Rigidity (GP)
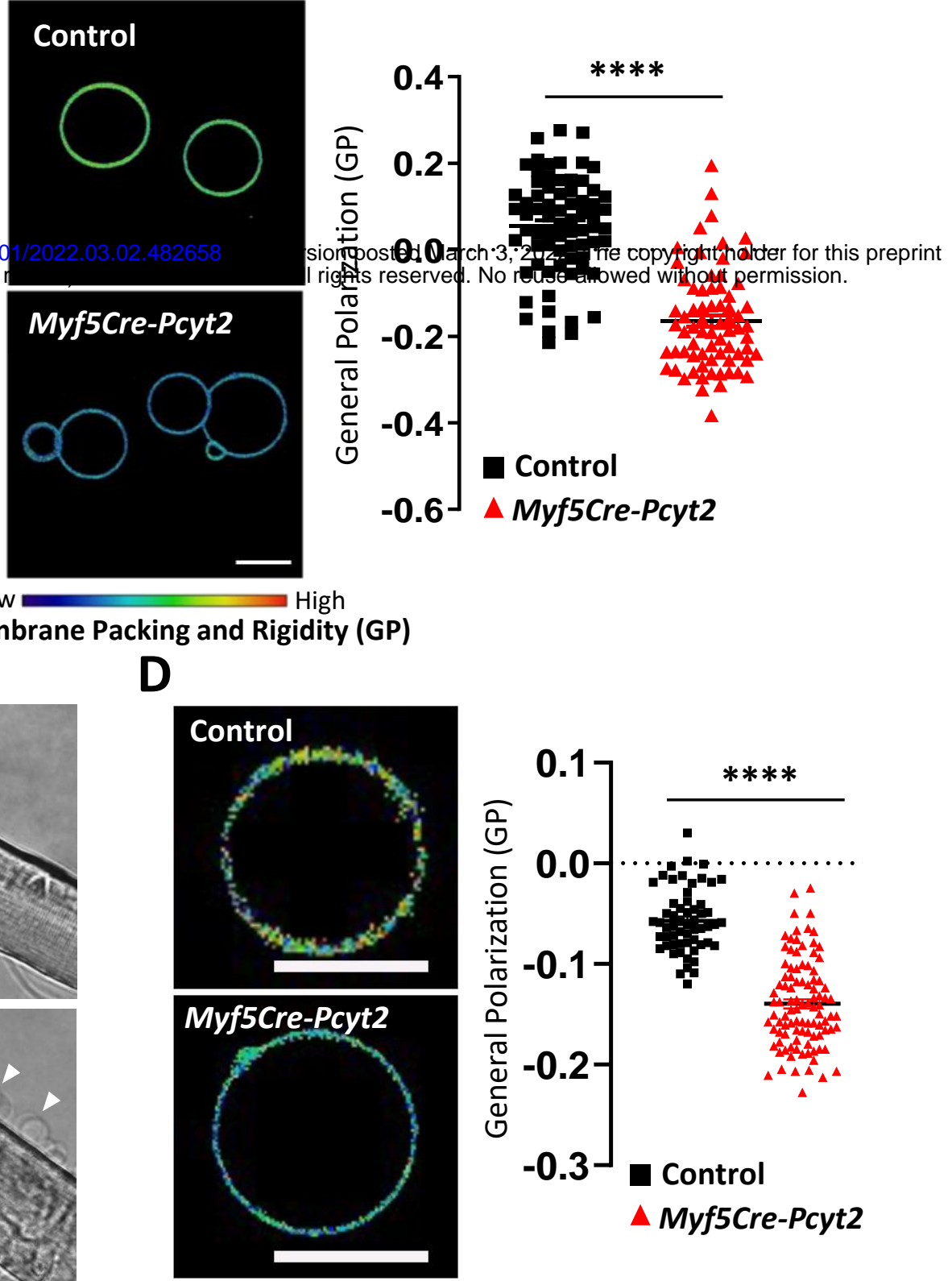

Myf5Cre-Pcyt2

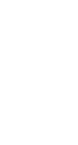

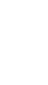


Figure 4

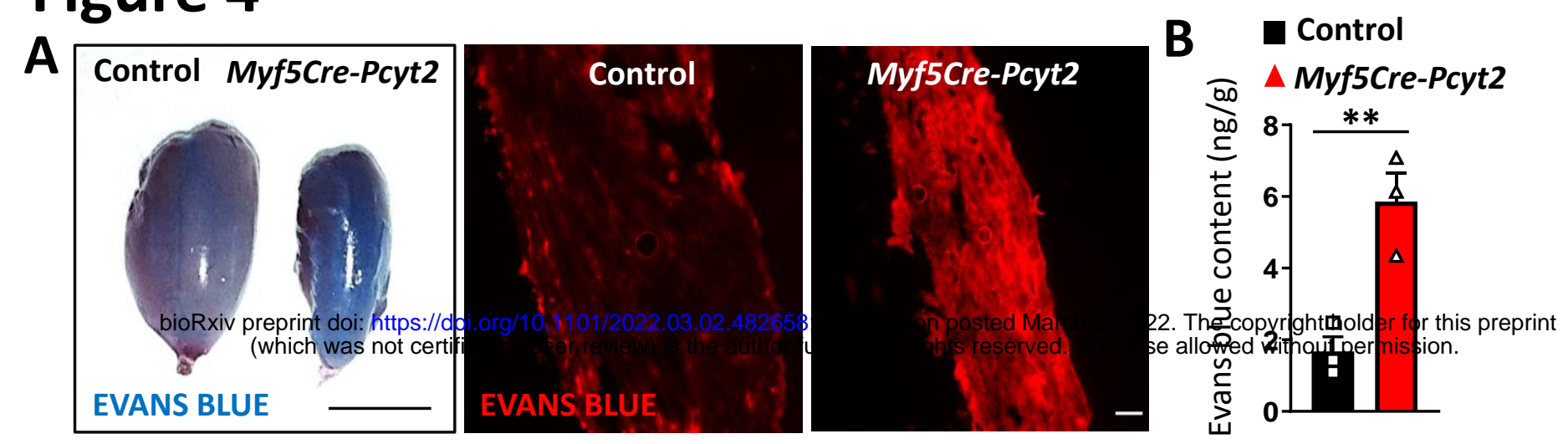

C
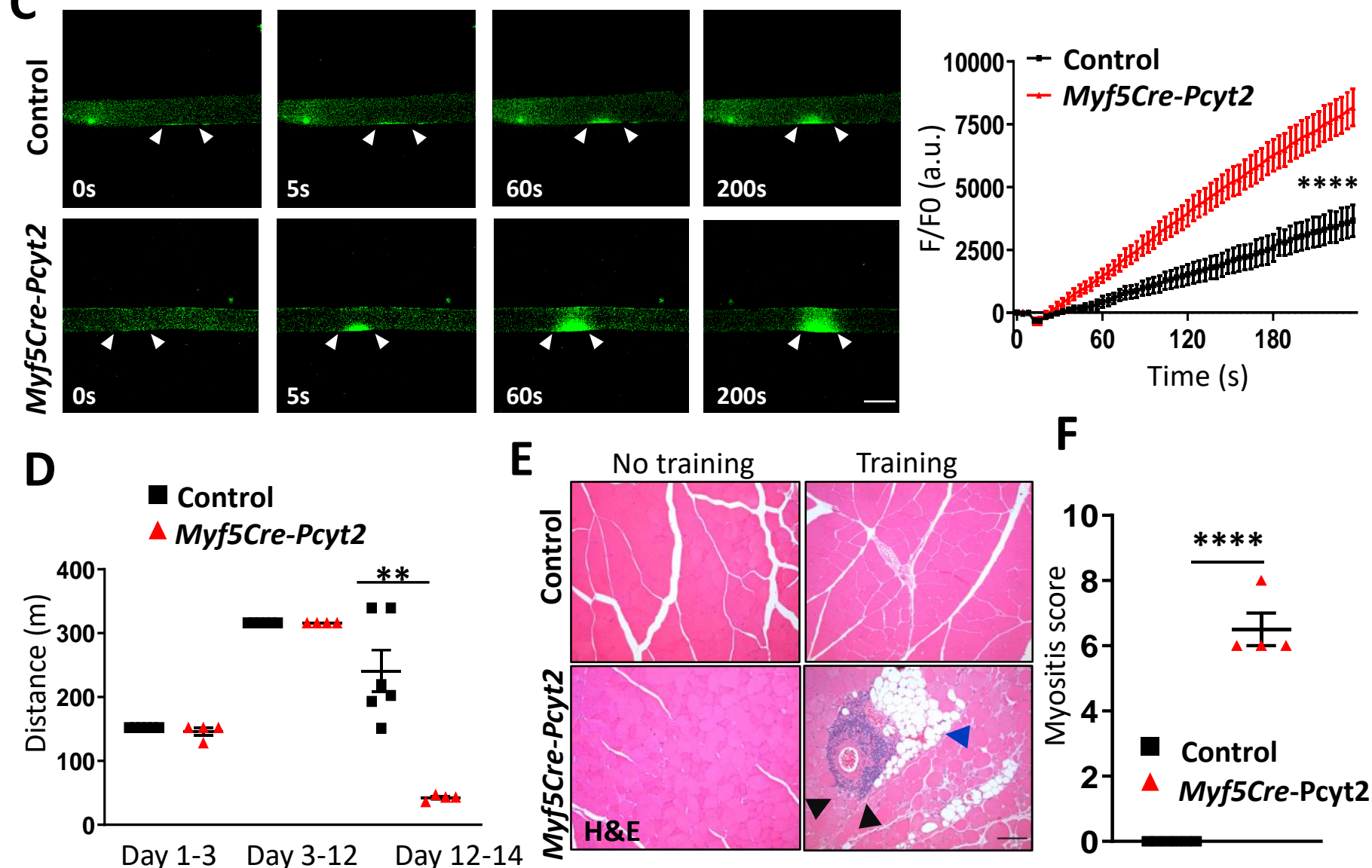

E No training

Training

F
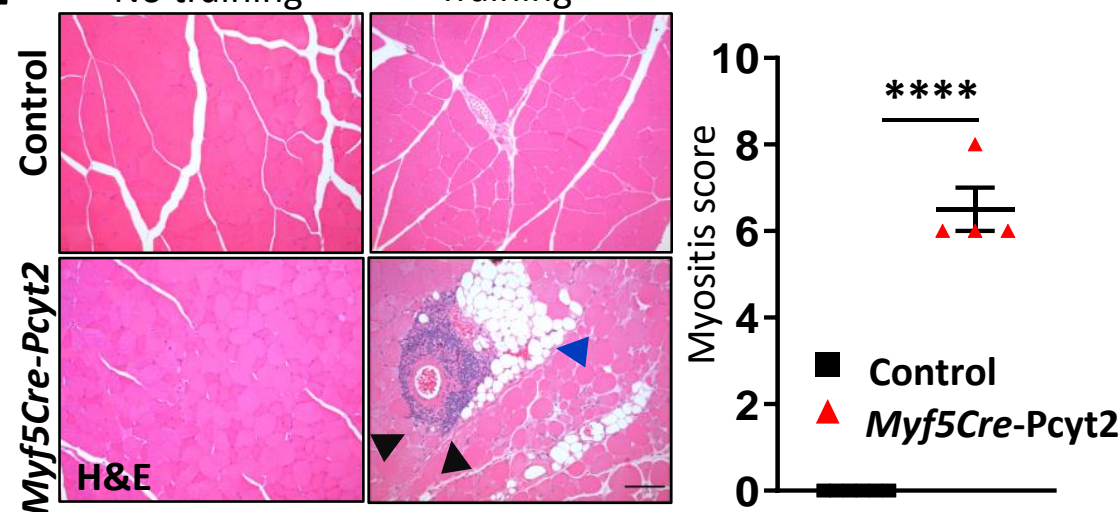

G

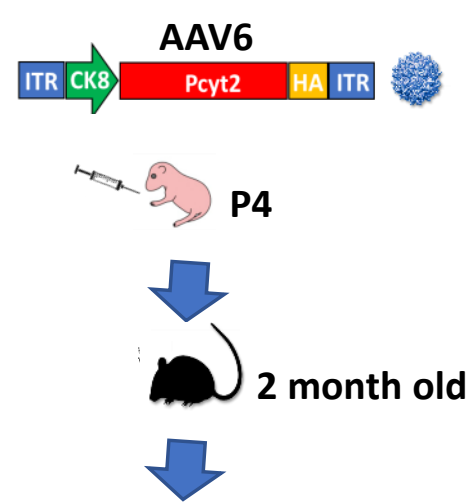

Grip Strength
$\mathrm{H}$

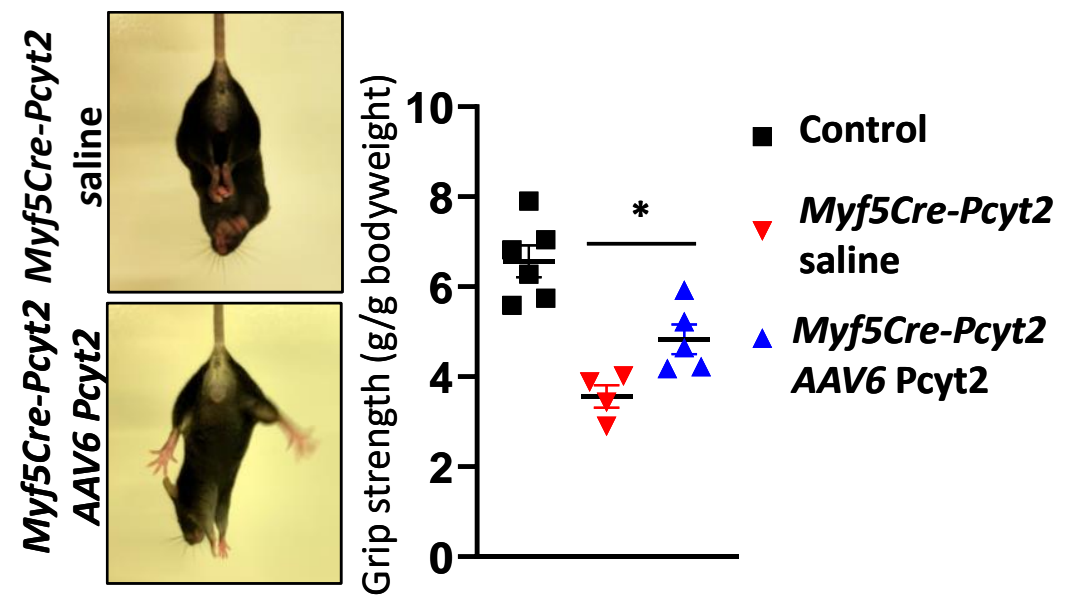

Control

$\Delta$ Pre-sarcopenic

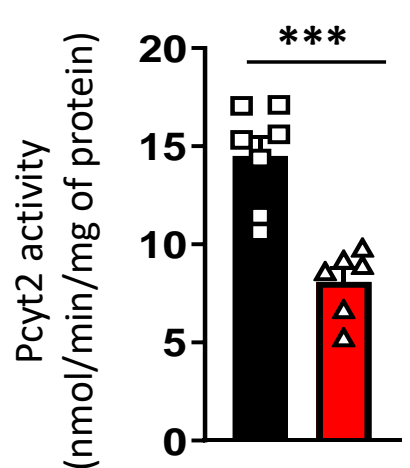

\section{J}

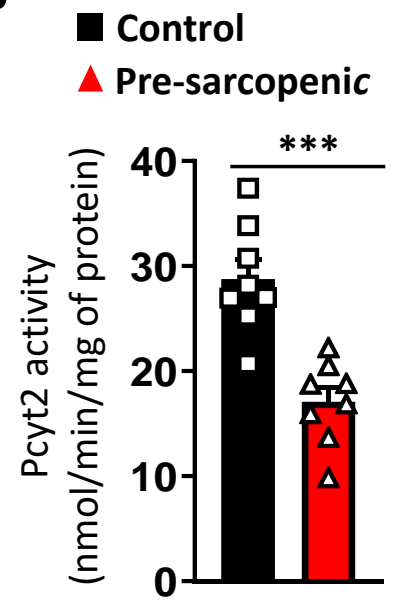

K
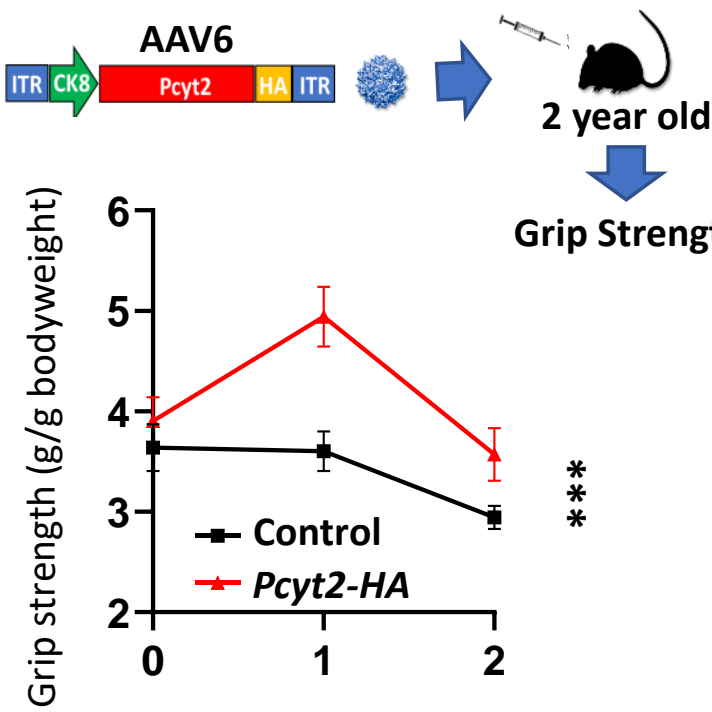

Time after gene therapy (months) 


\section{Extended Data Figure 1}

A

Ethanolamine
biokxiv preprint doi:

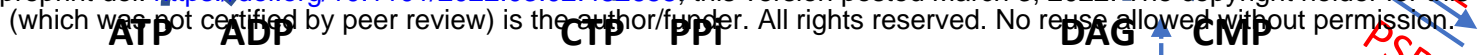

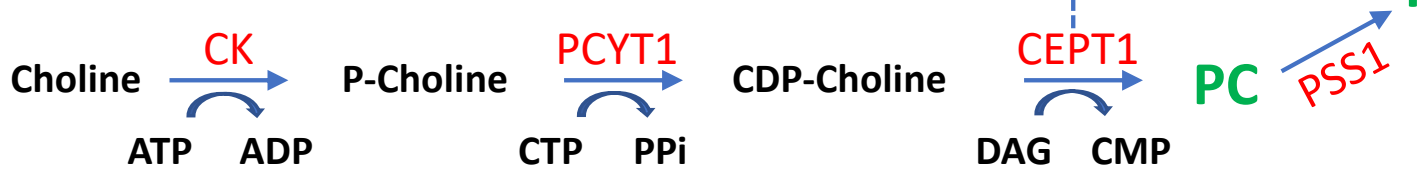

B

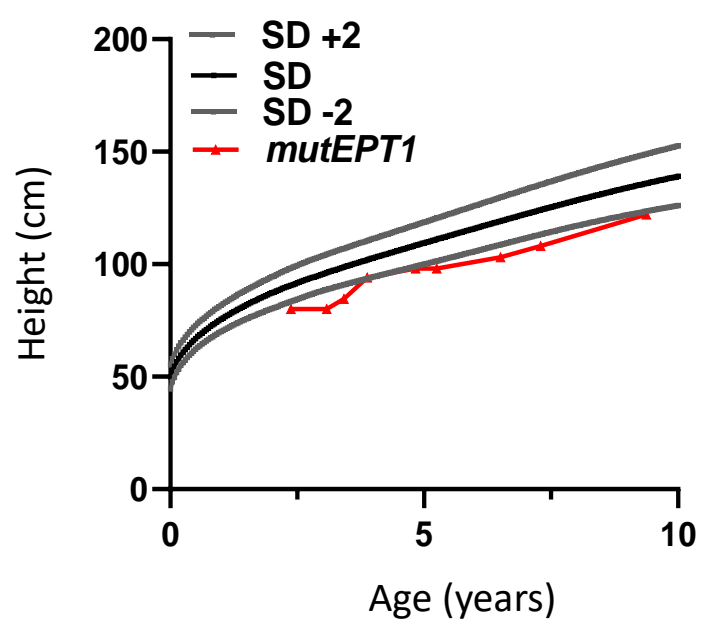

\section{Patient \#1}
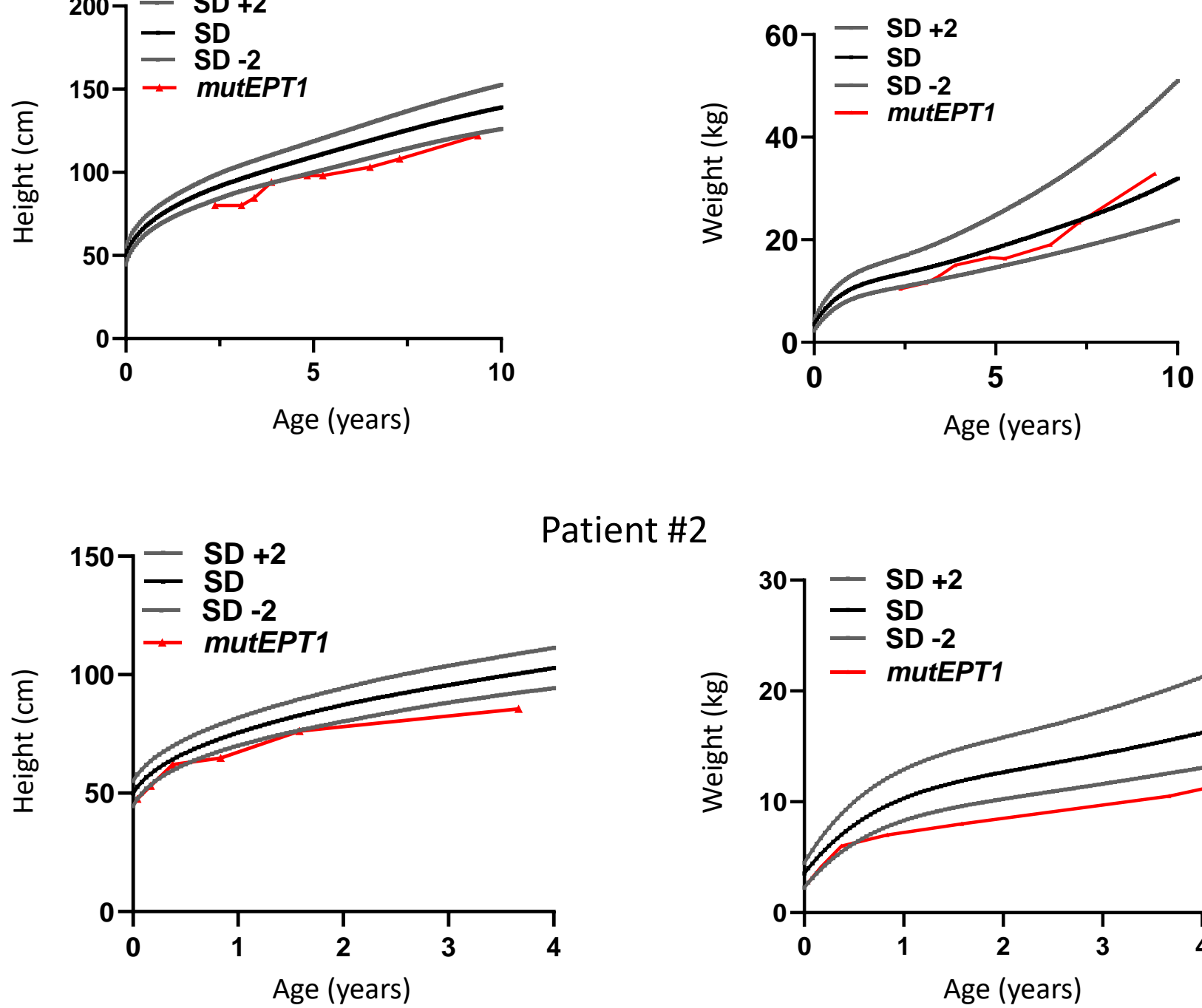

Patient \#2
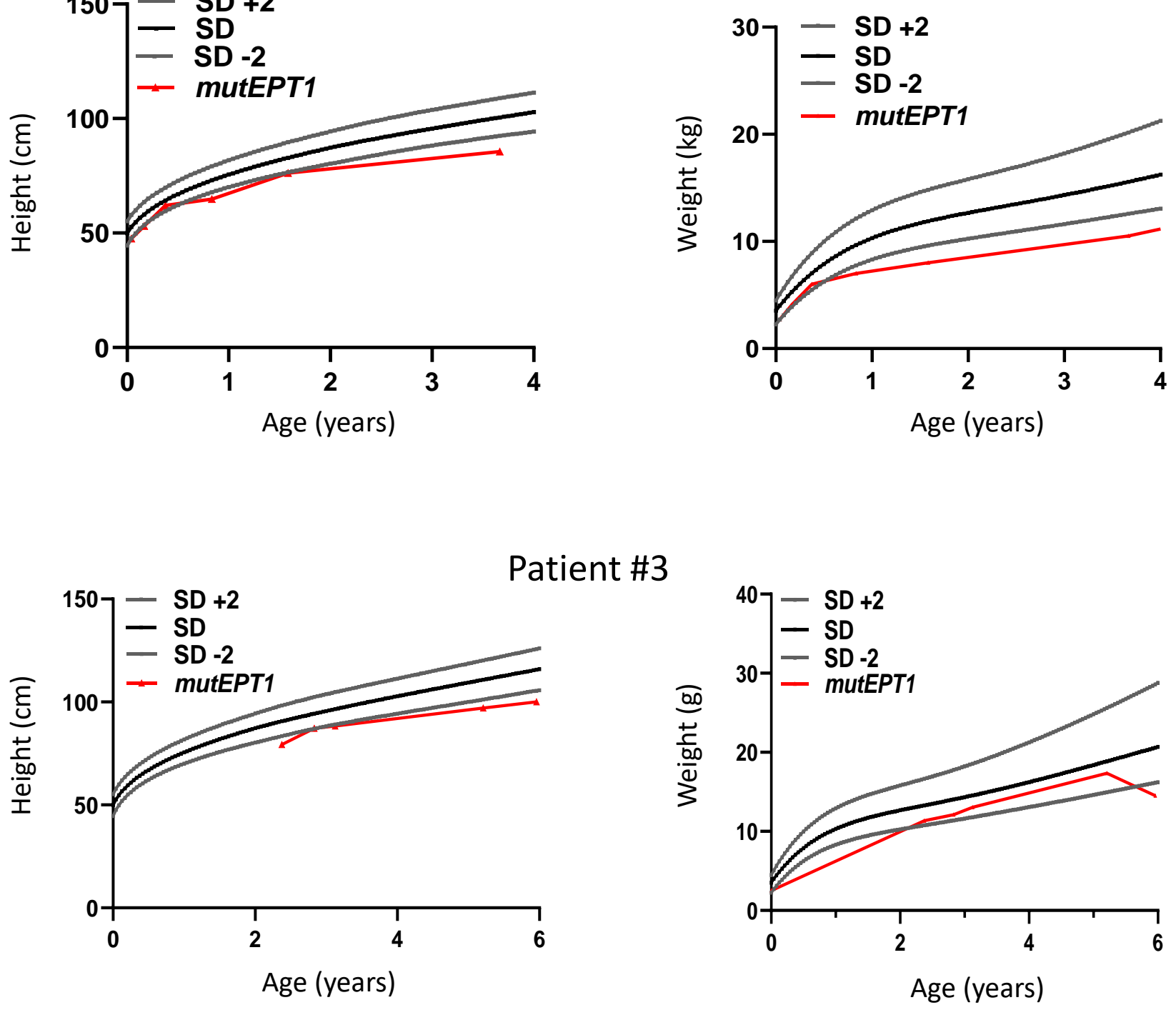
+1 bioRxiv preprint doi: https://doi.org/10.1101/2022.03.089482658; this version posted March 3, 2022. The ct37/right holder for this preprint

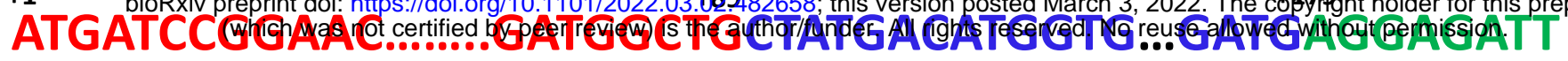

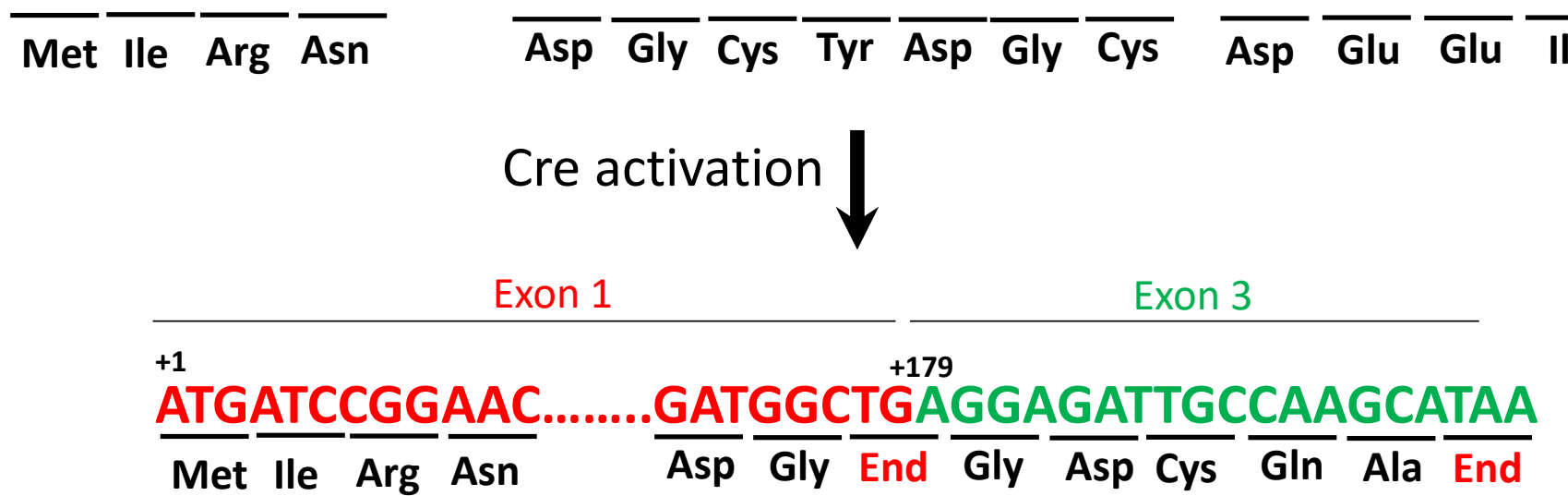

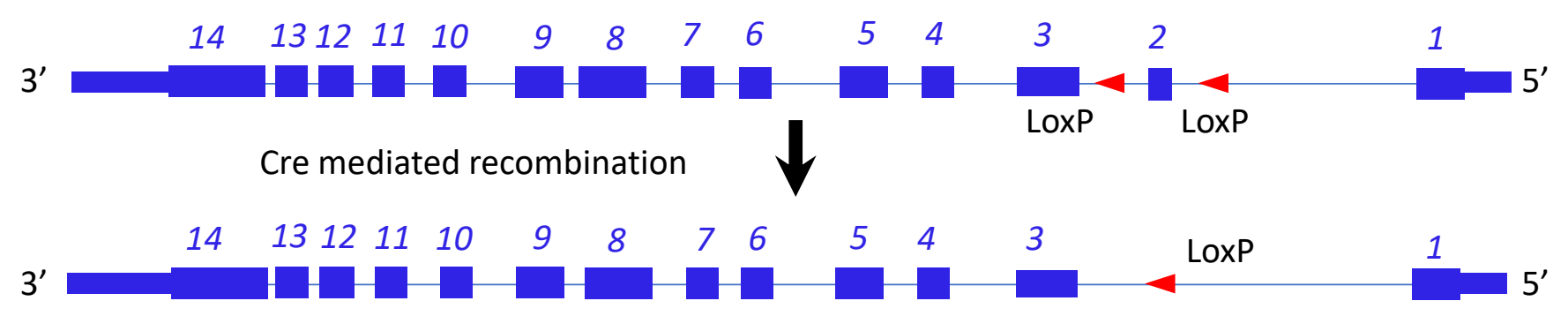

Pcyt2 genomic locus

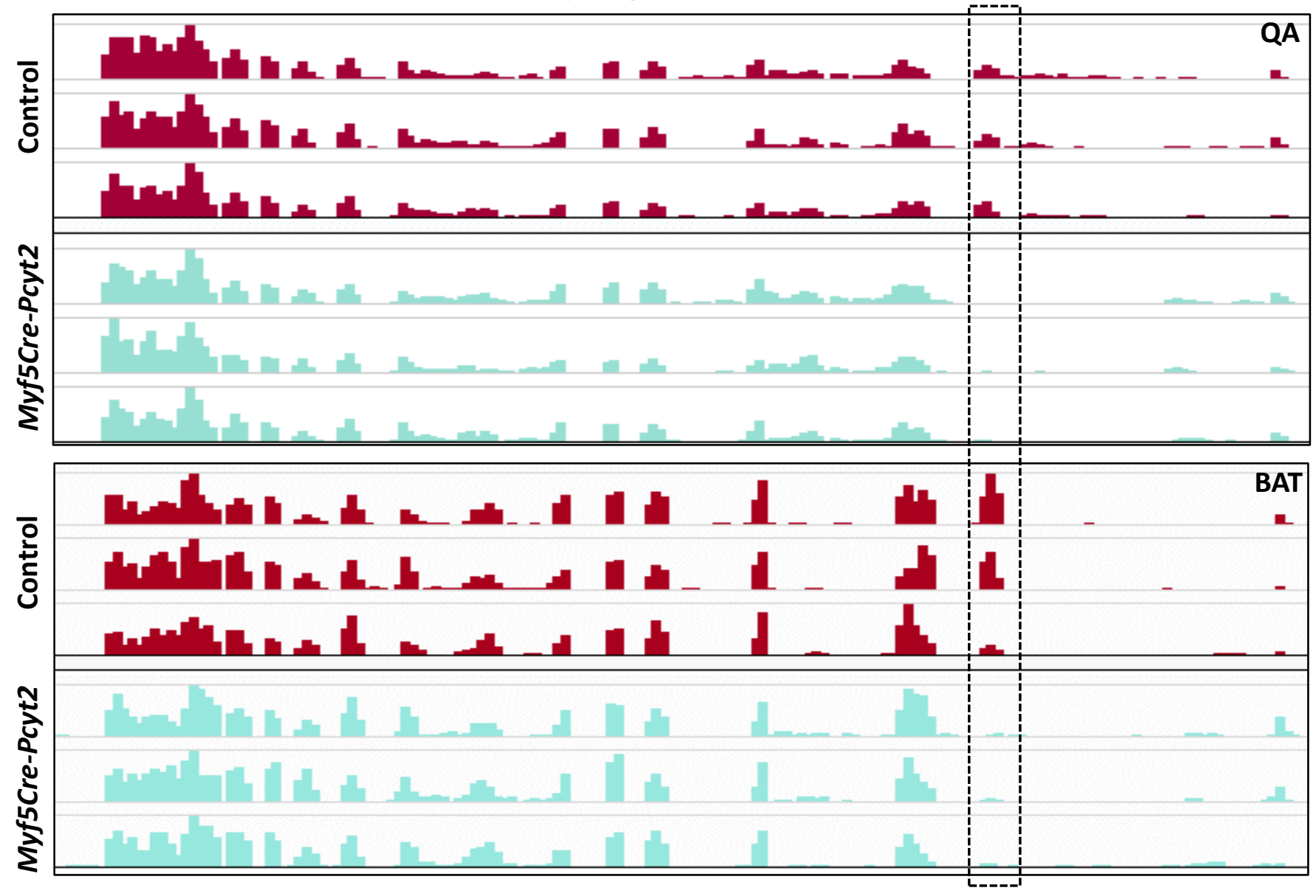


Extended Data Figure 3

A
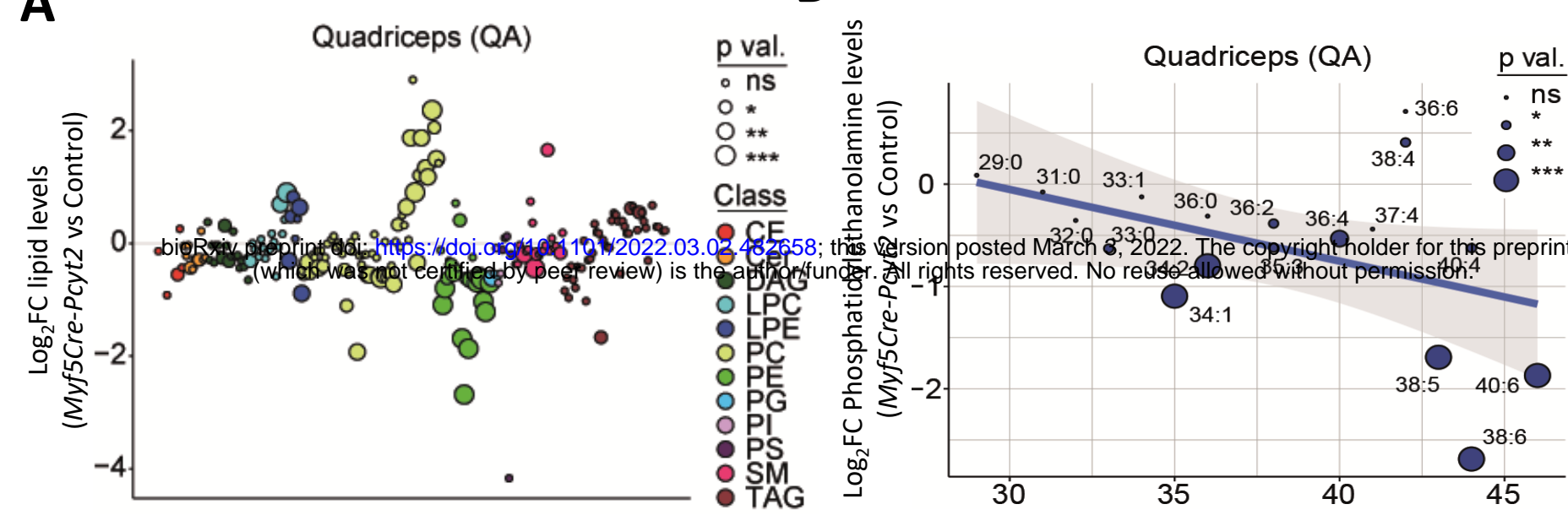

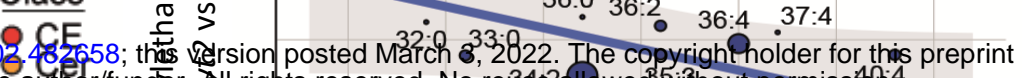

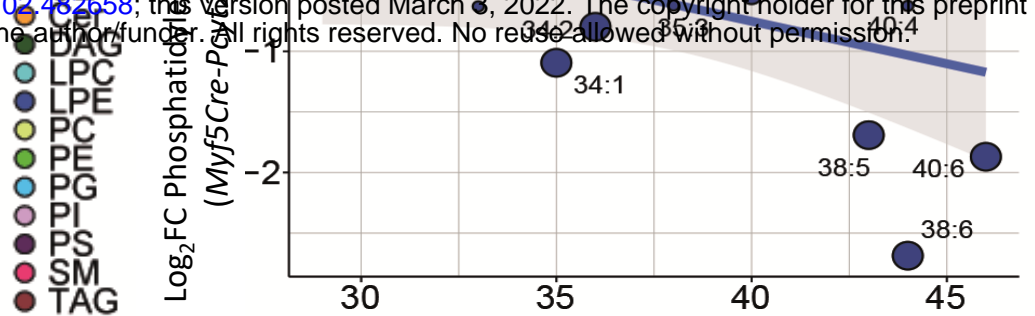

C

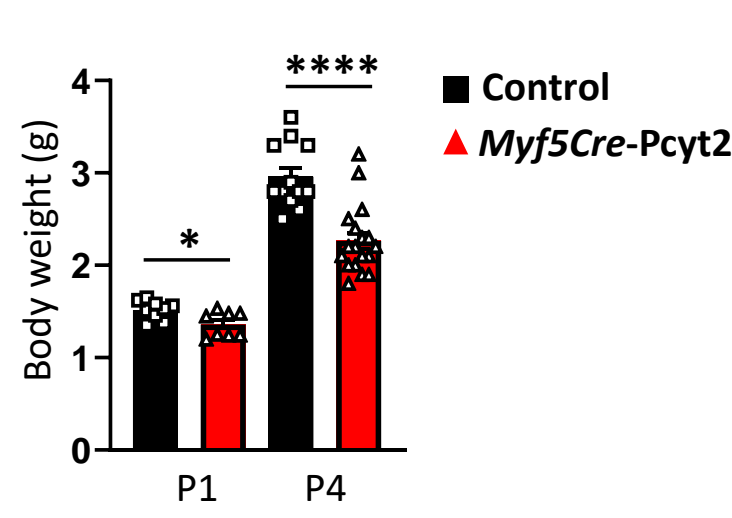

D

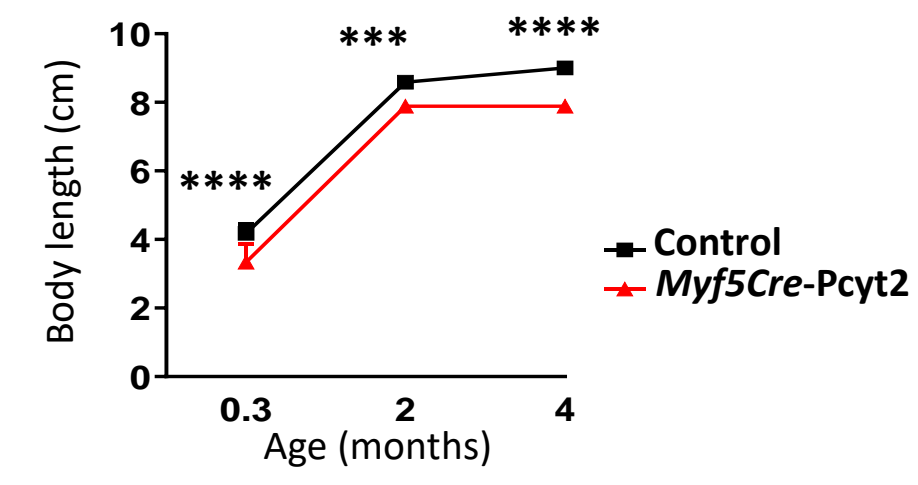

$\mathbf{E}$

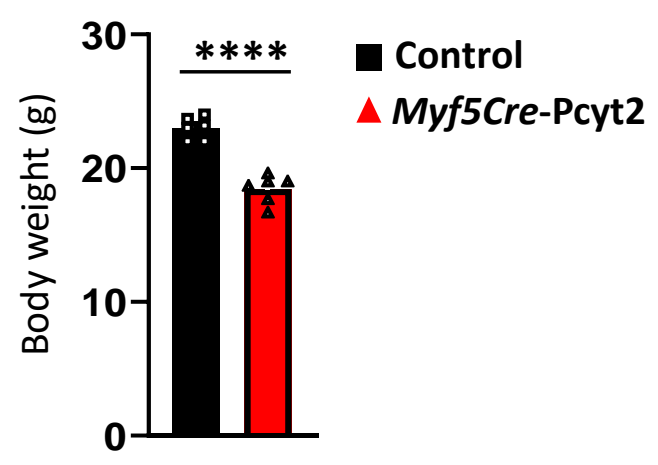

$\boldsymbol{F}$

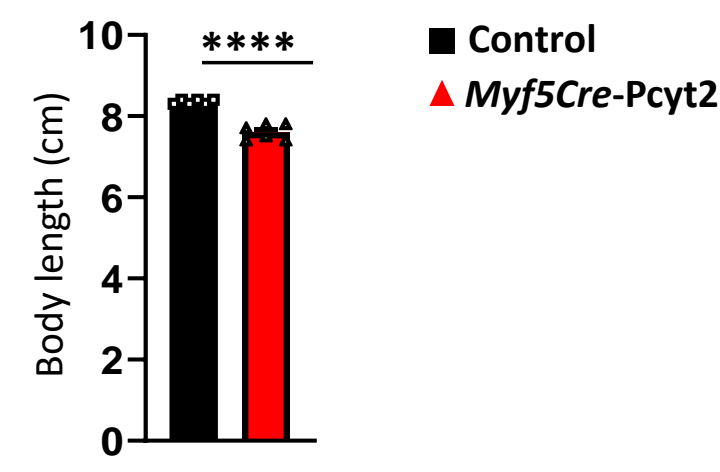

$\mathbf{G}$

Control

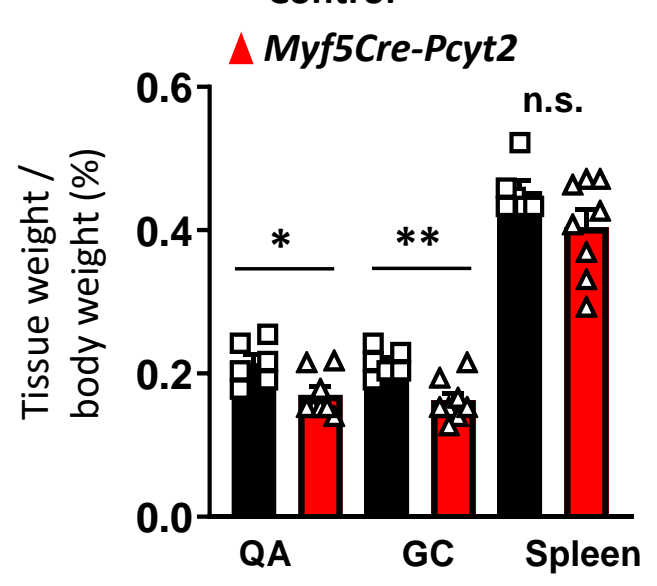

H

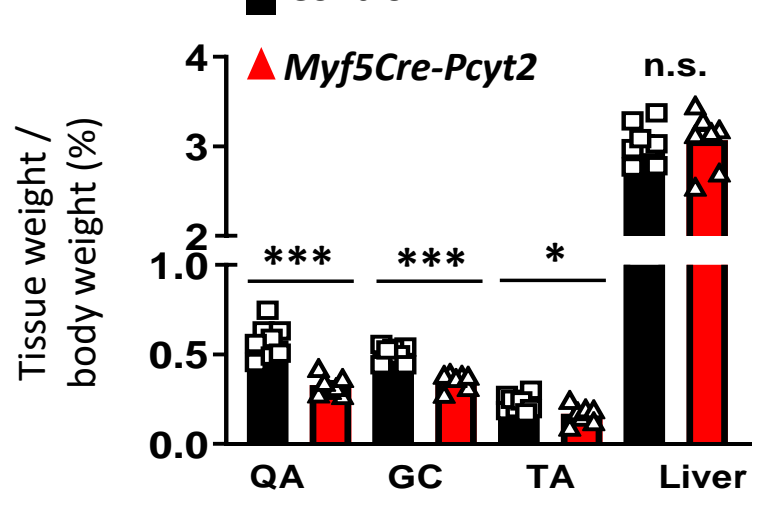

J
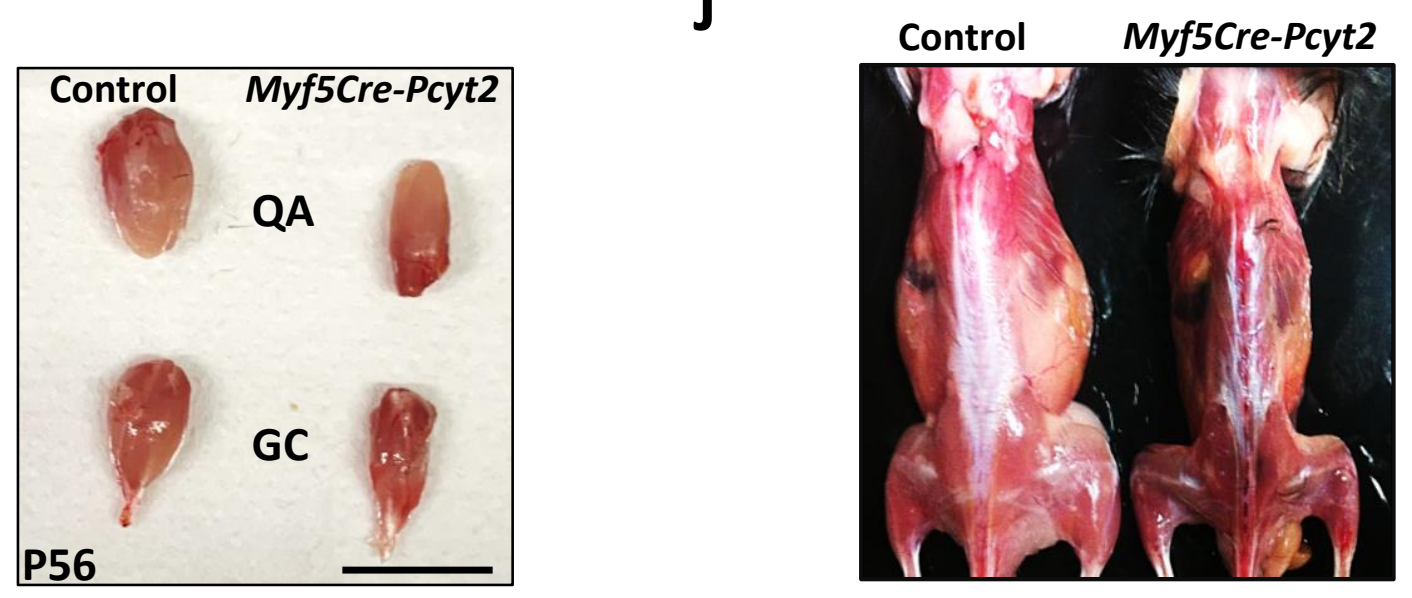


\section{Extended Data Figure 4}

A

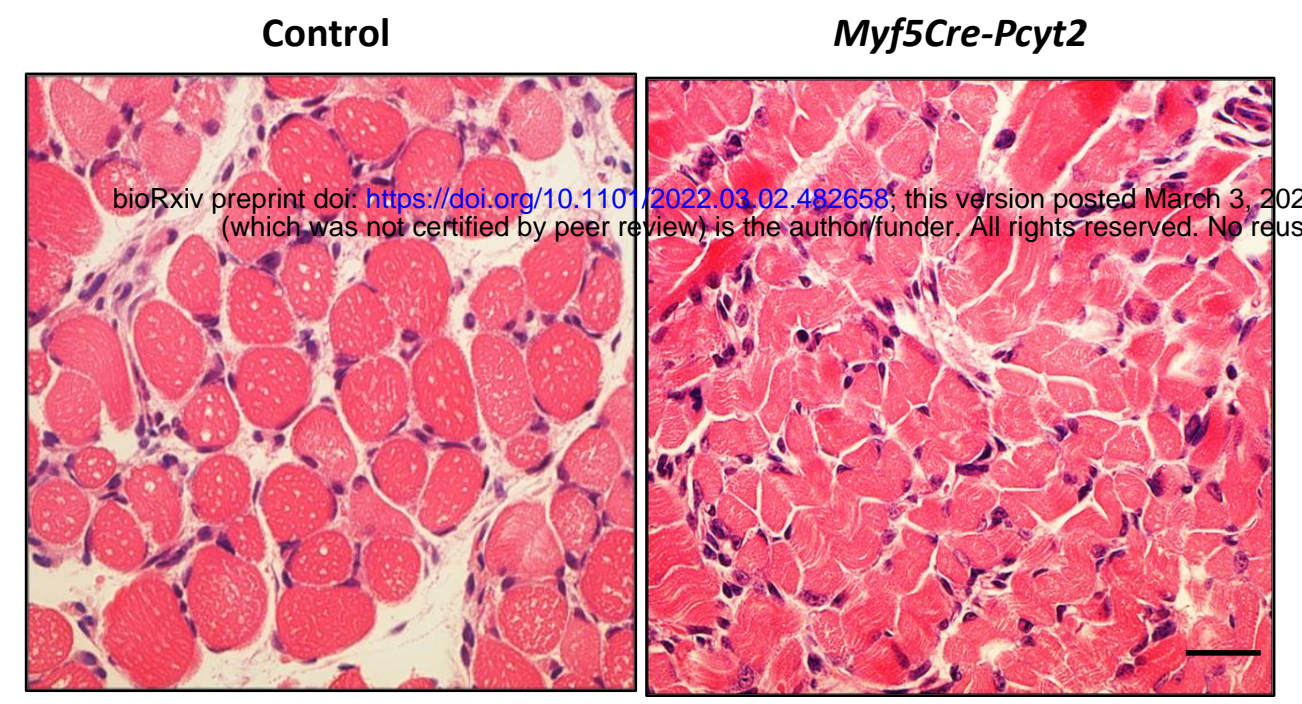

B
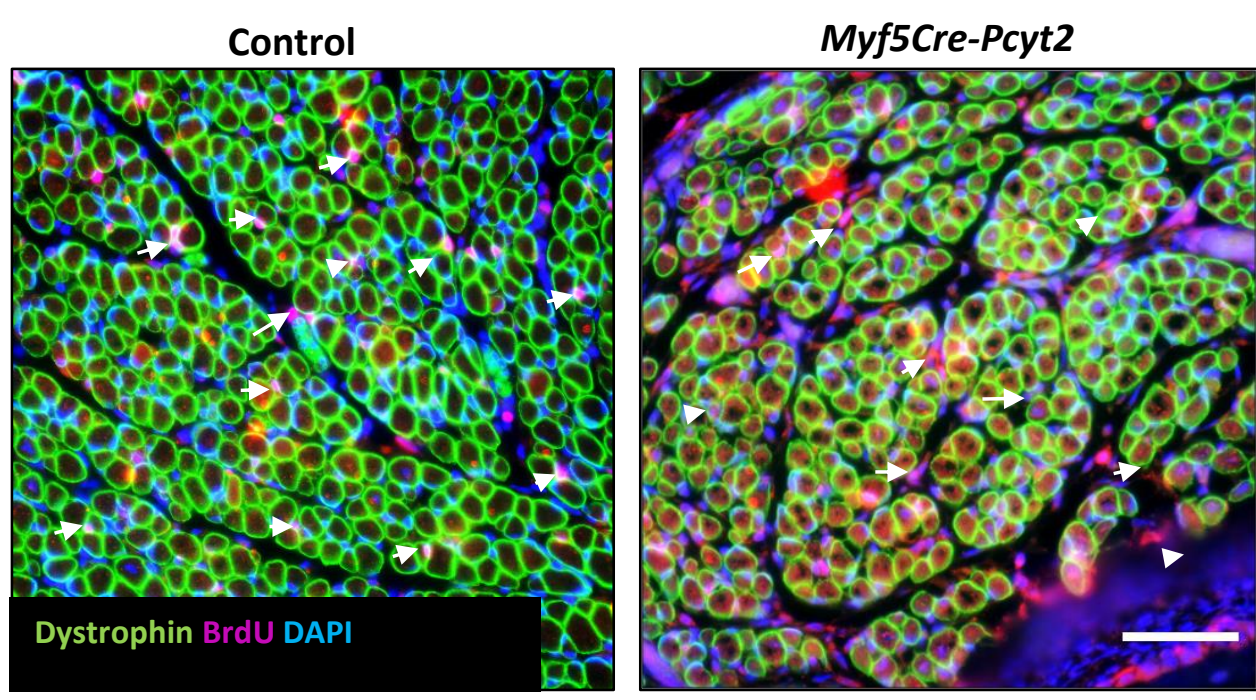

C
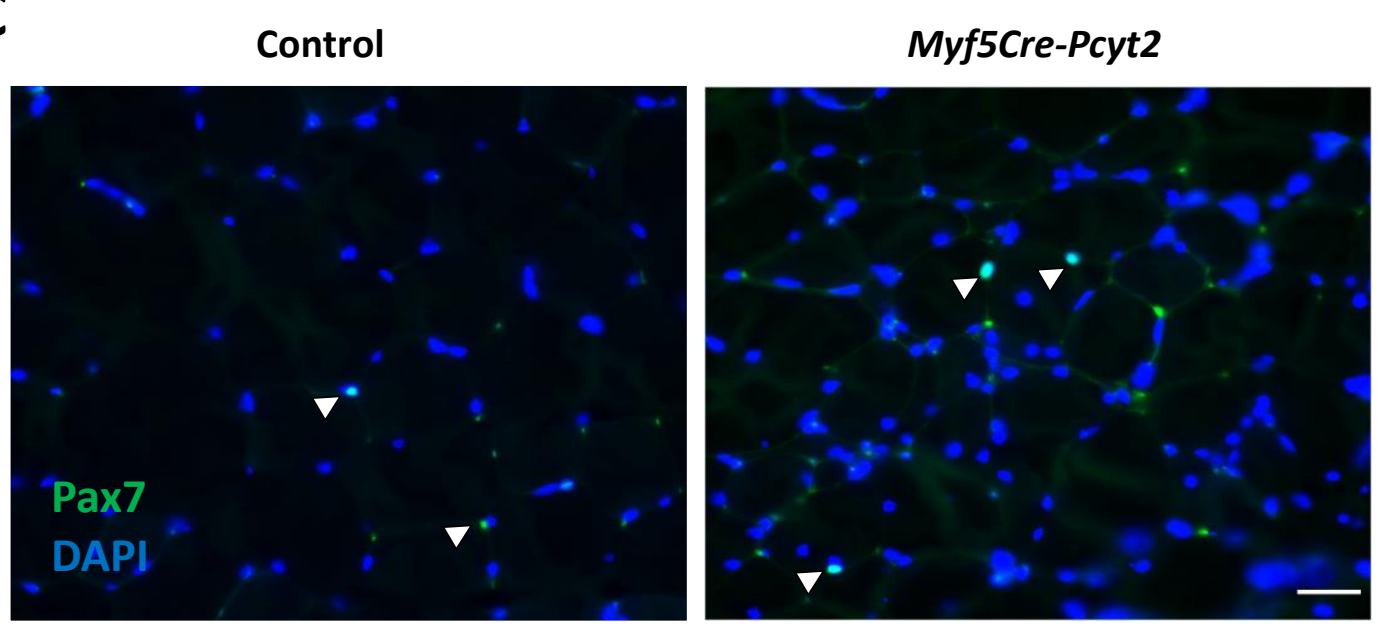

- Control

Myf5Cre-Pcyt2

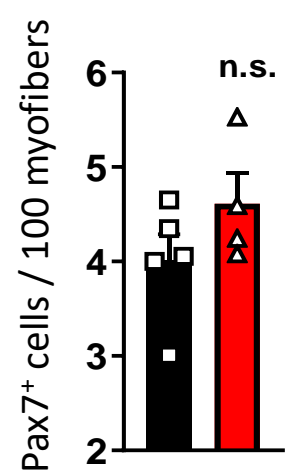

D

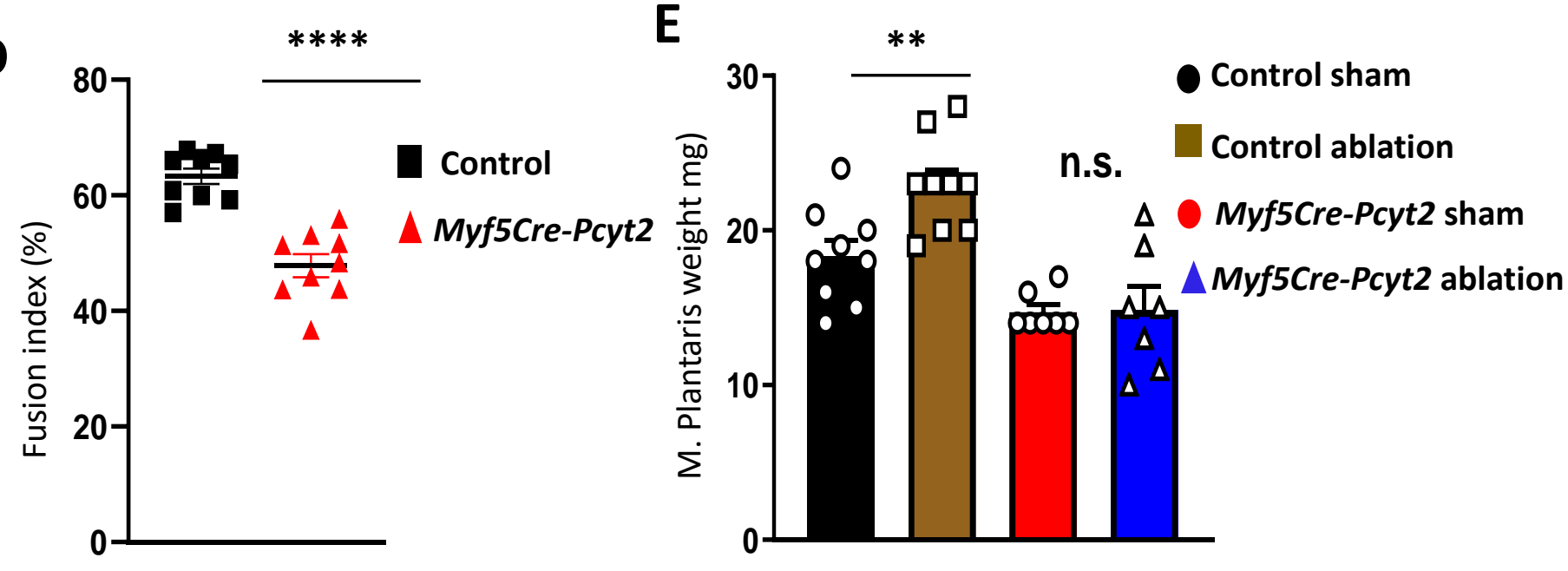




\section{Extended Data Figure 5}

\section{A}

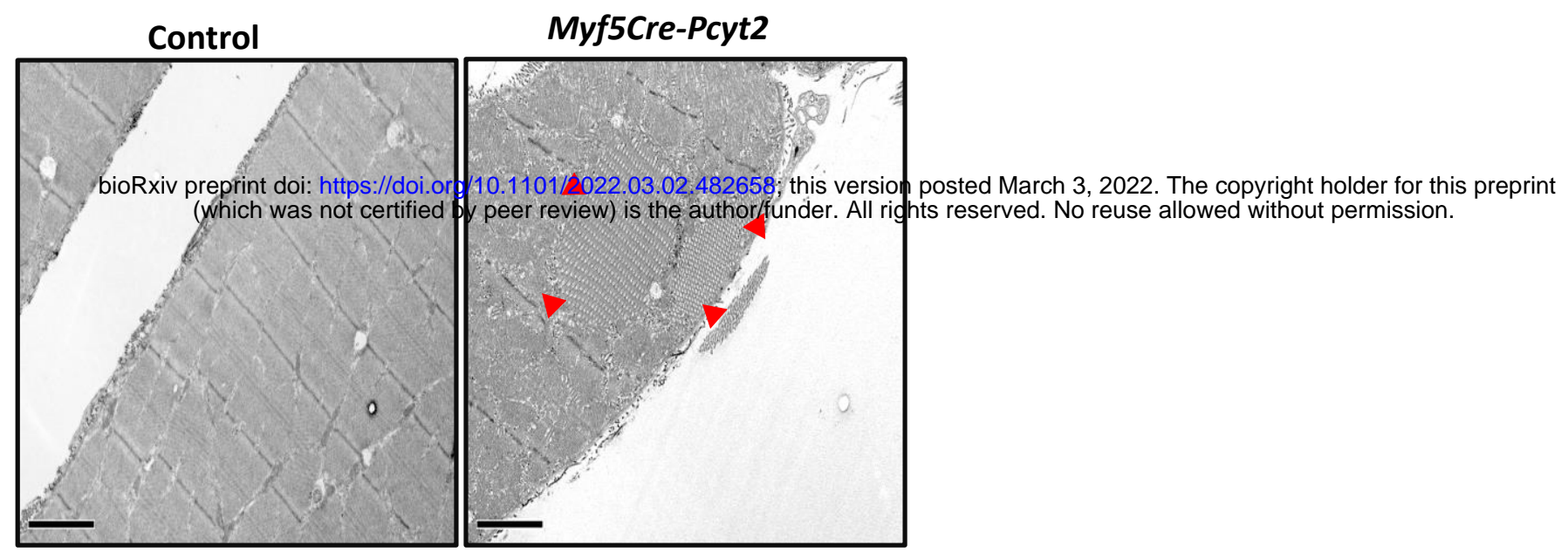

B

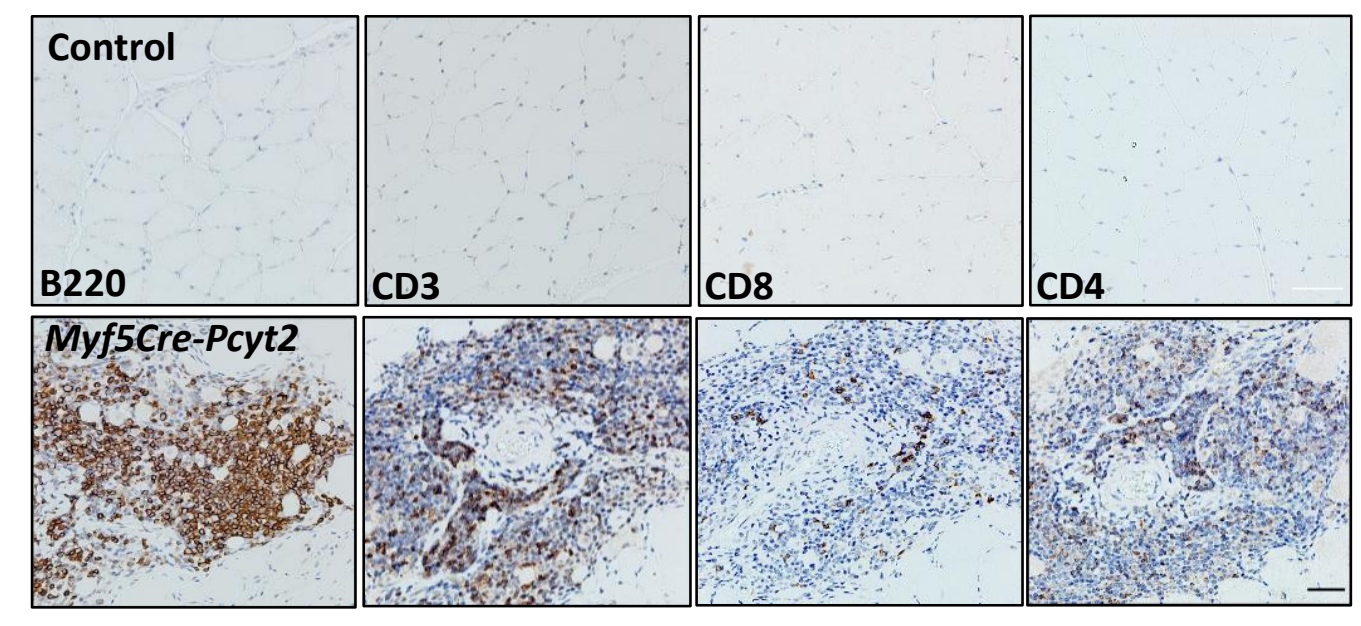

D

- Control $\triangle$ Myf5Cre-Pcyt2

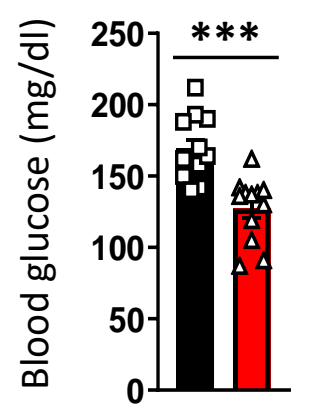

G

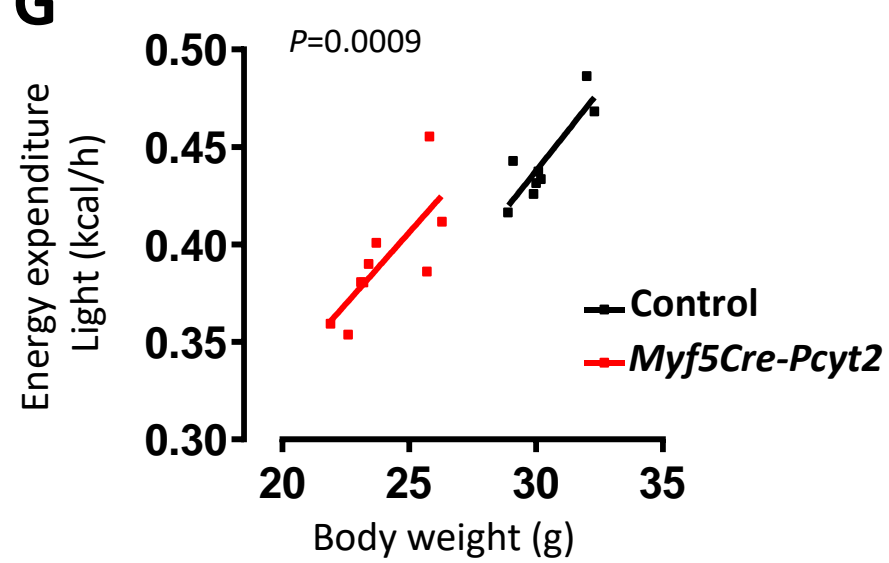

H c

Control

Myf5Cre-Pcyt2

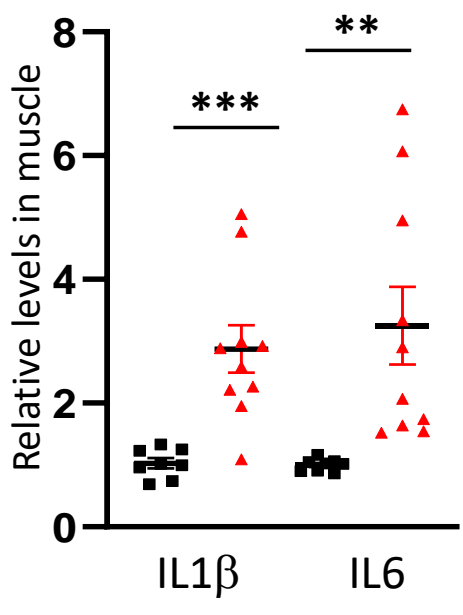

E

- Control

F

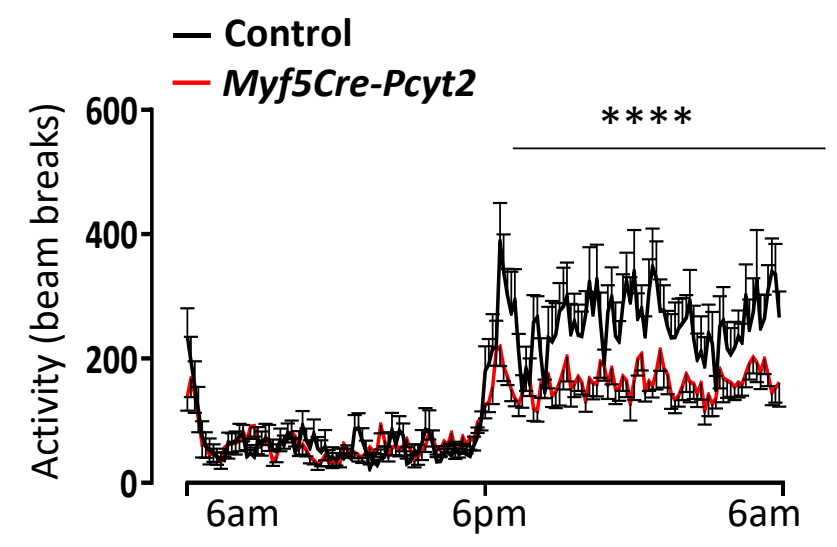

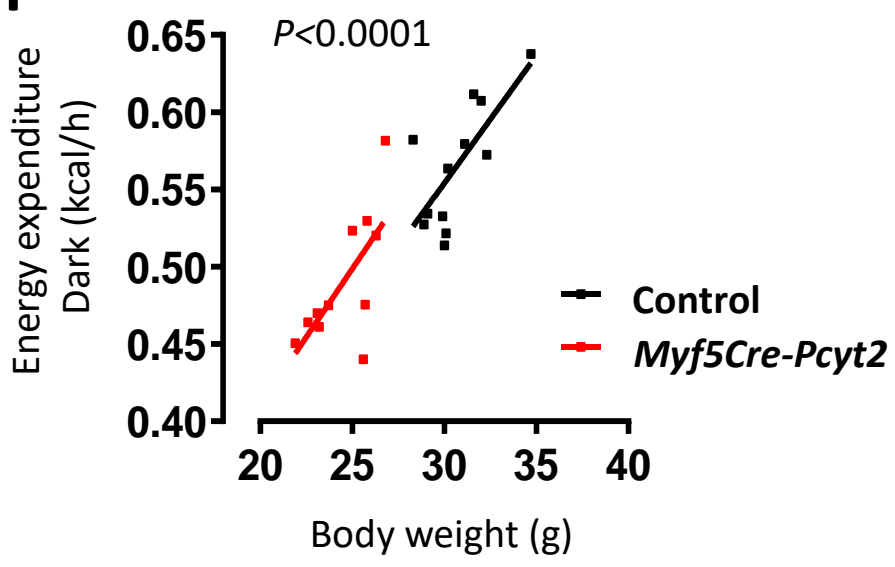




\section{Extended Data Figure 6}

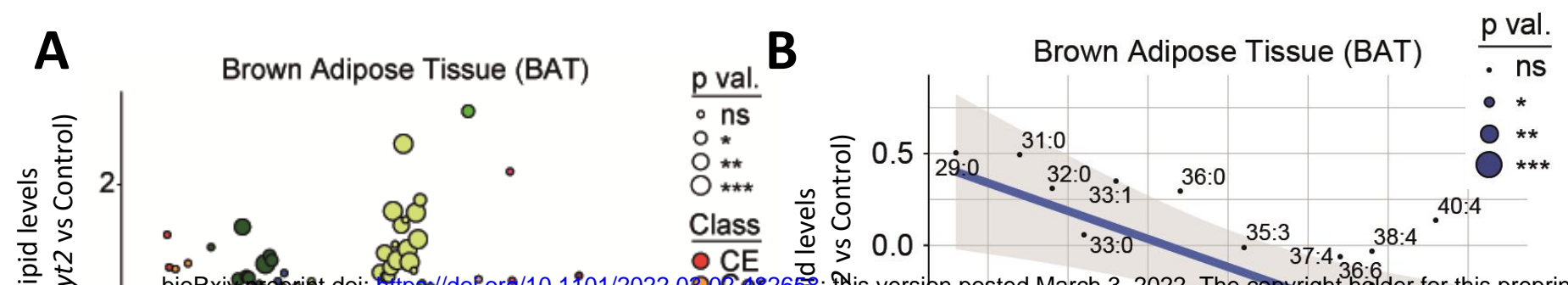

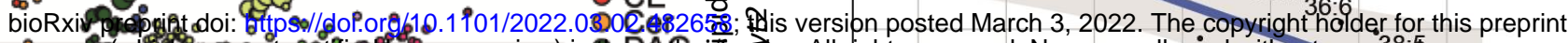
(whit

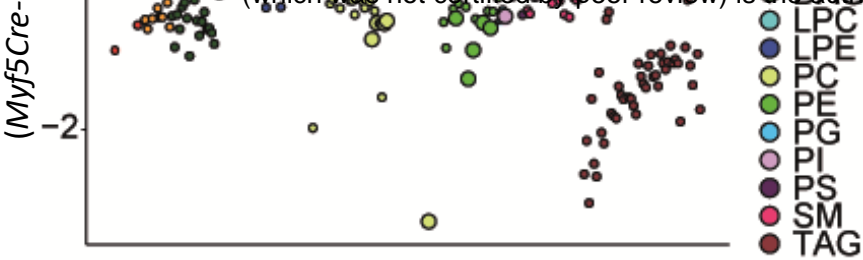

C
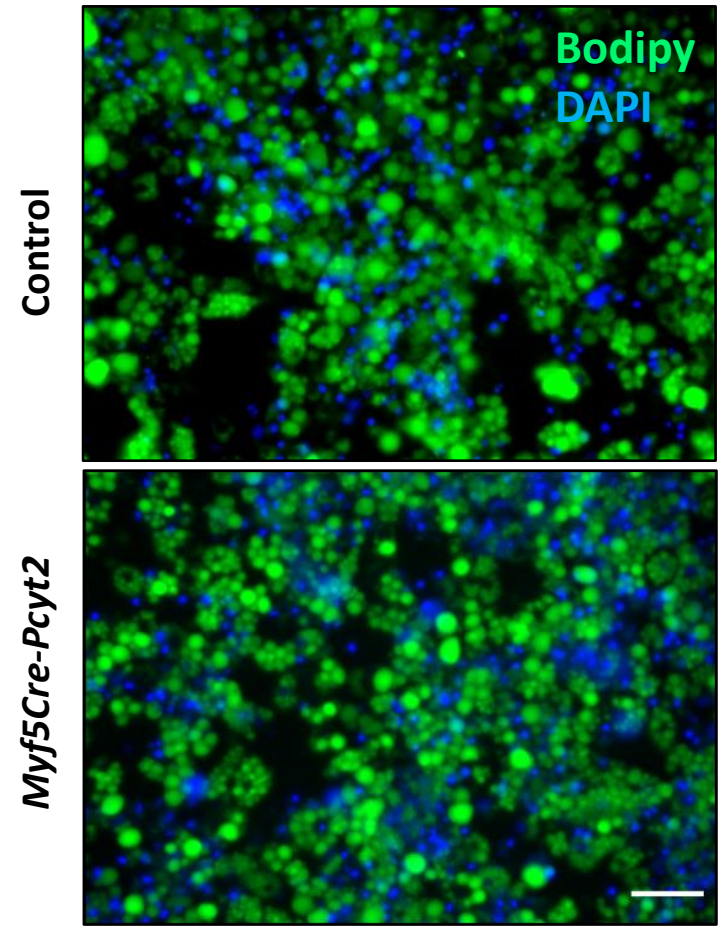

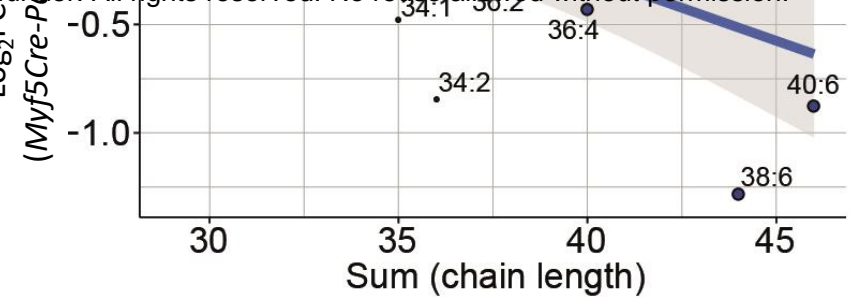

D

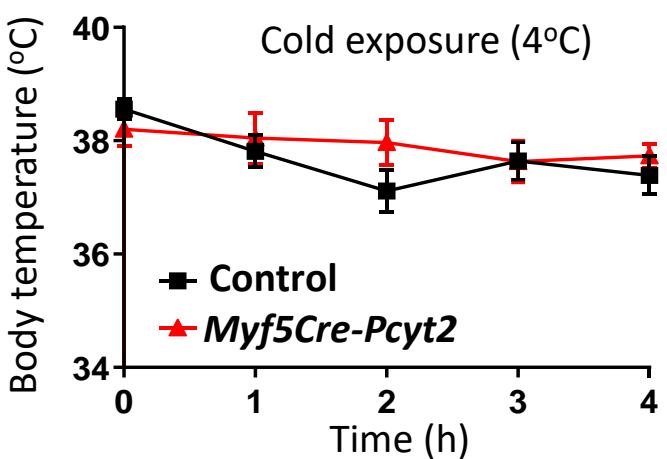

E

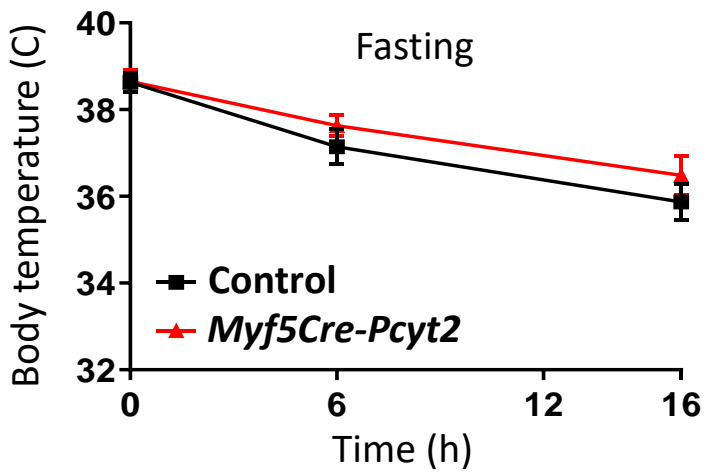

H

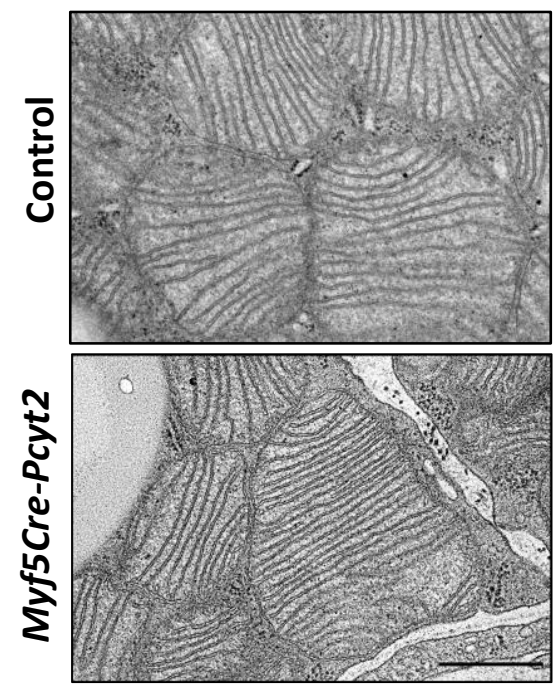

Control

$\triangle$ Myf5Cre-Pcyt2

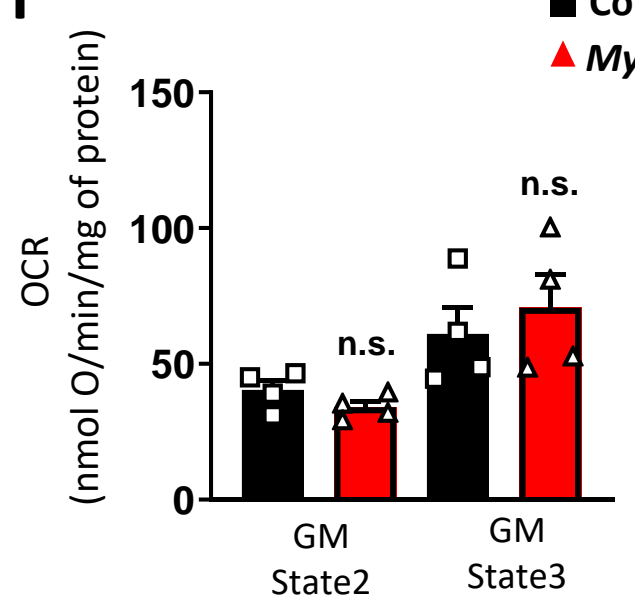

J

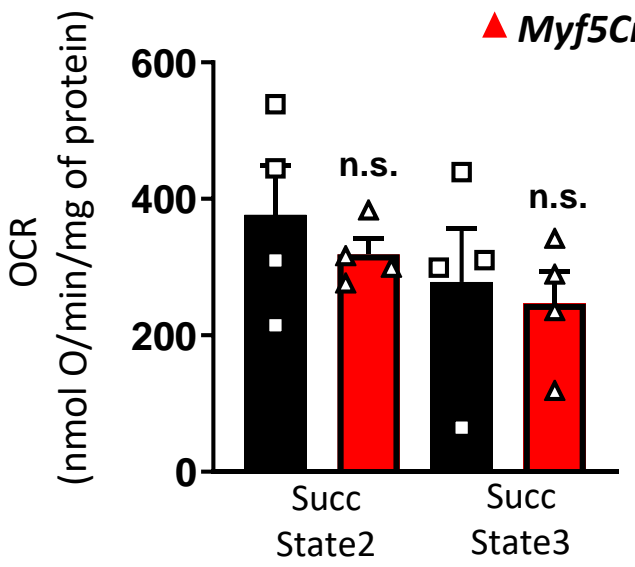




\section{Extended Data Figure 7}

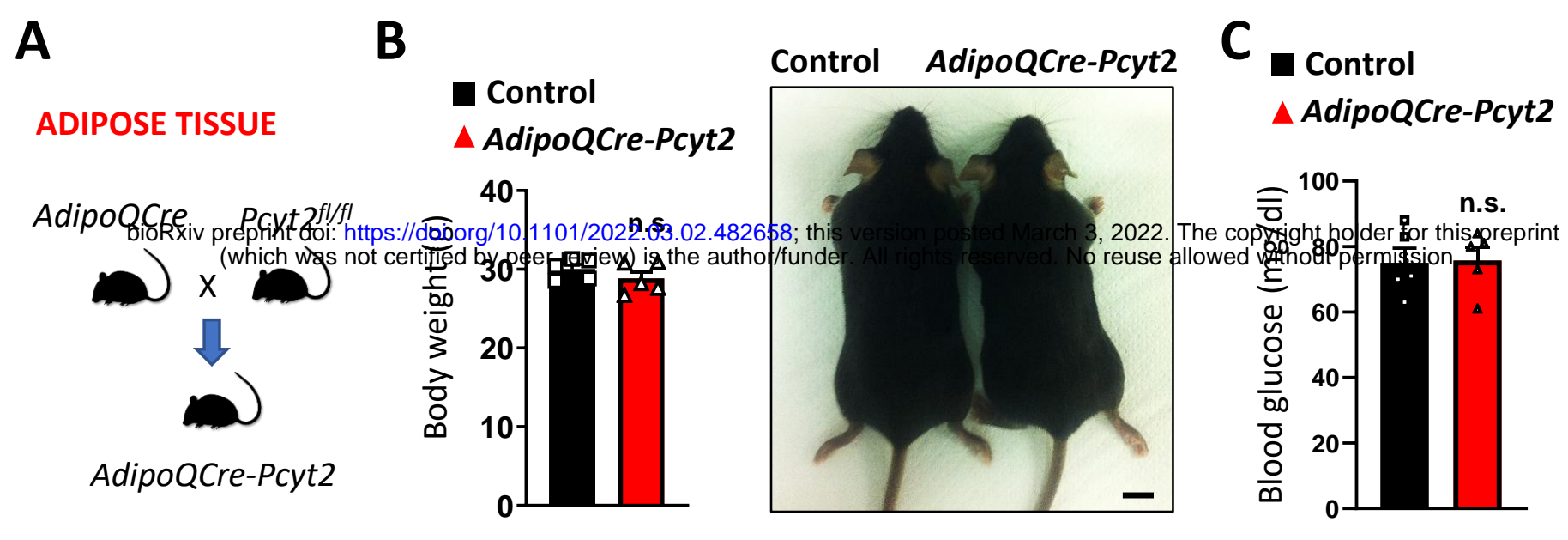

D

MOTOR NEURONS

Mnx1 Cre Pcyt2fl/fl

D $\times$

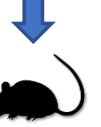

Mnx1Cre-Pcyt2

G

E
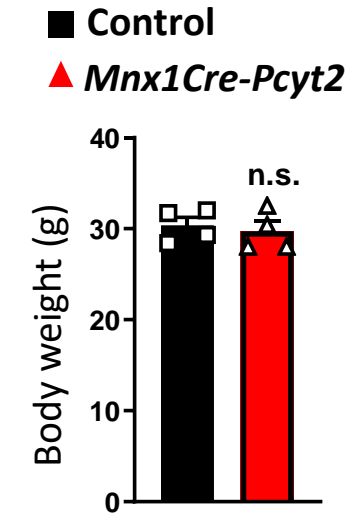

F Control Mnx1Cre-Pcyt2

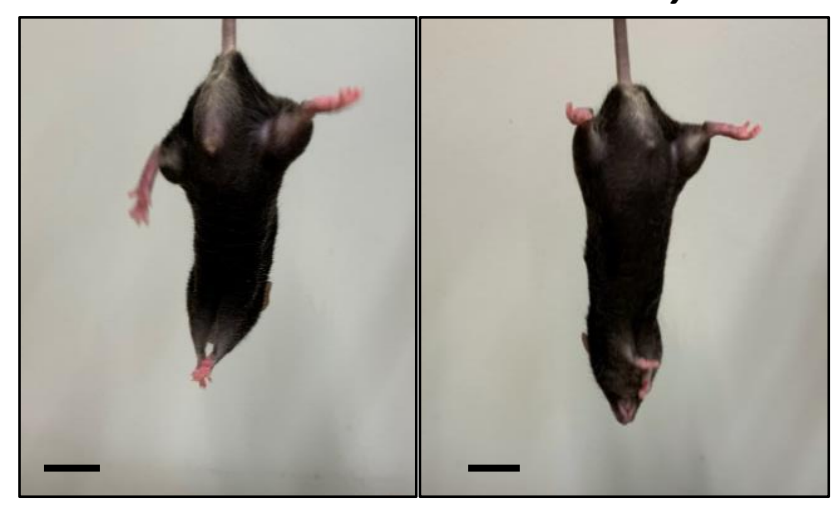

H

- Control

INTESTINE

$\Delta$ Villin1Cre-Pcyt2

Villin1Cre $\quad$ Pcyt $2^{f / f l}$

D $\times$

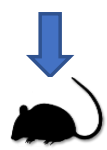

Villin1Cre-Pcyt2
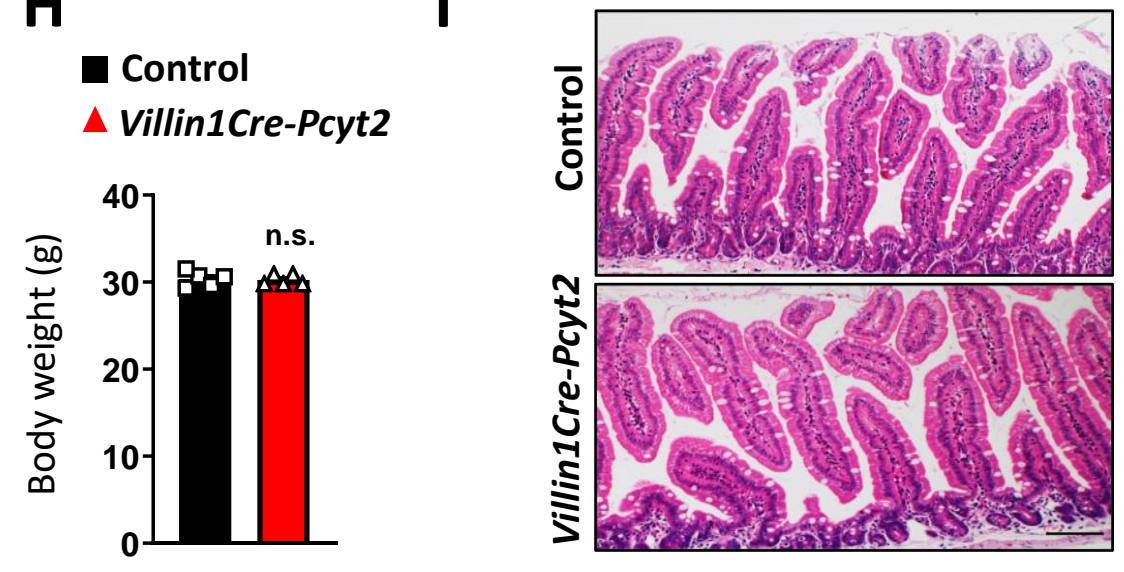

K

J

SKIN

K14Cre Pcyt2fl/fl

D) $\times$
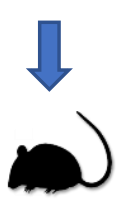

K14Cre-Pcyt2

M

MATURE MUSCLE
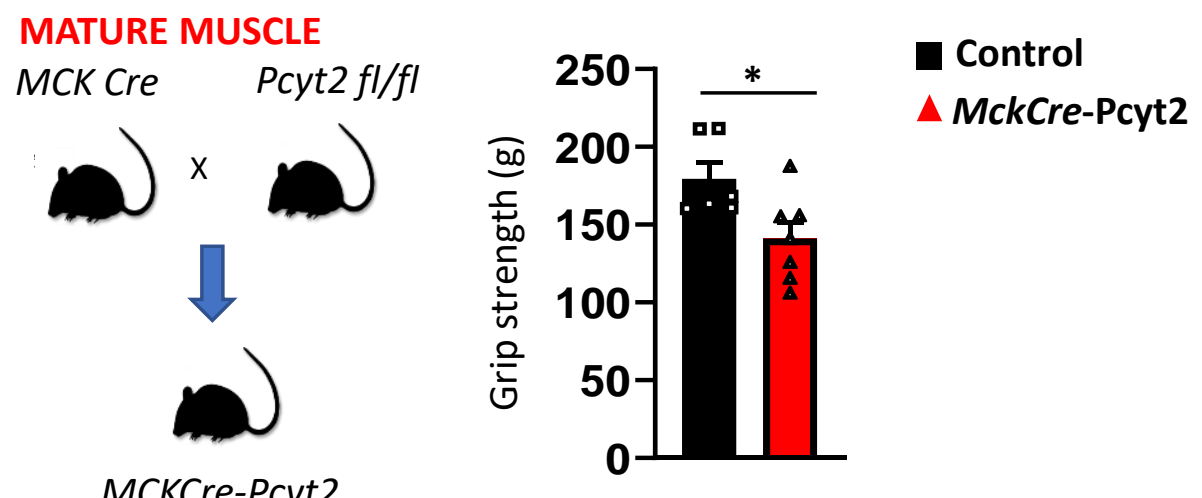

Control K14Cre-Pcyt2

- Control

$\triangle$ K14Cre-Pcyt2

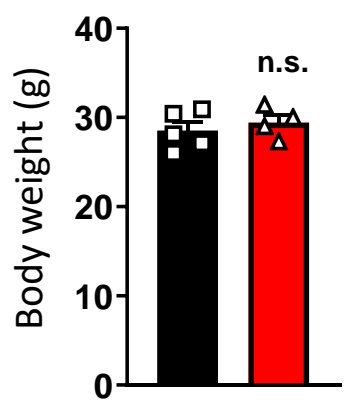

L

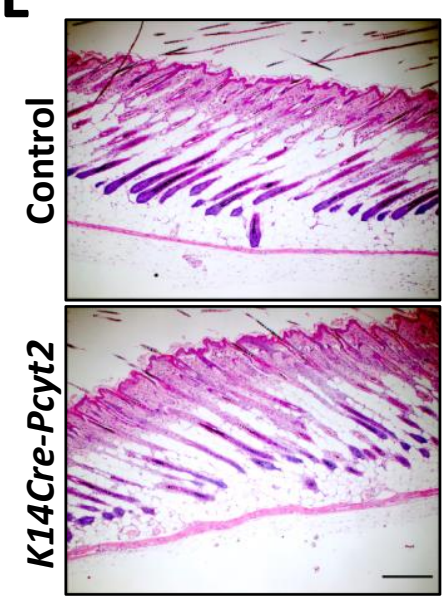

$\mathbf{N}$ 


\section{Extended Data Figure 8}
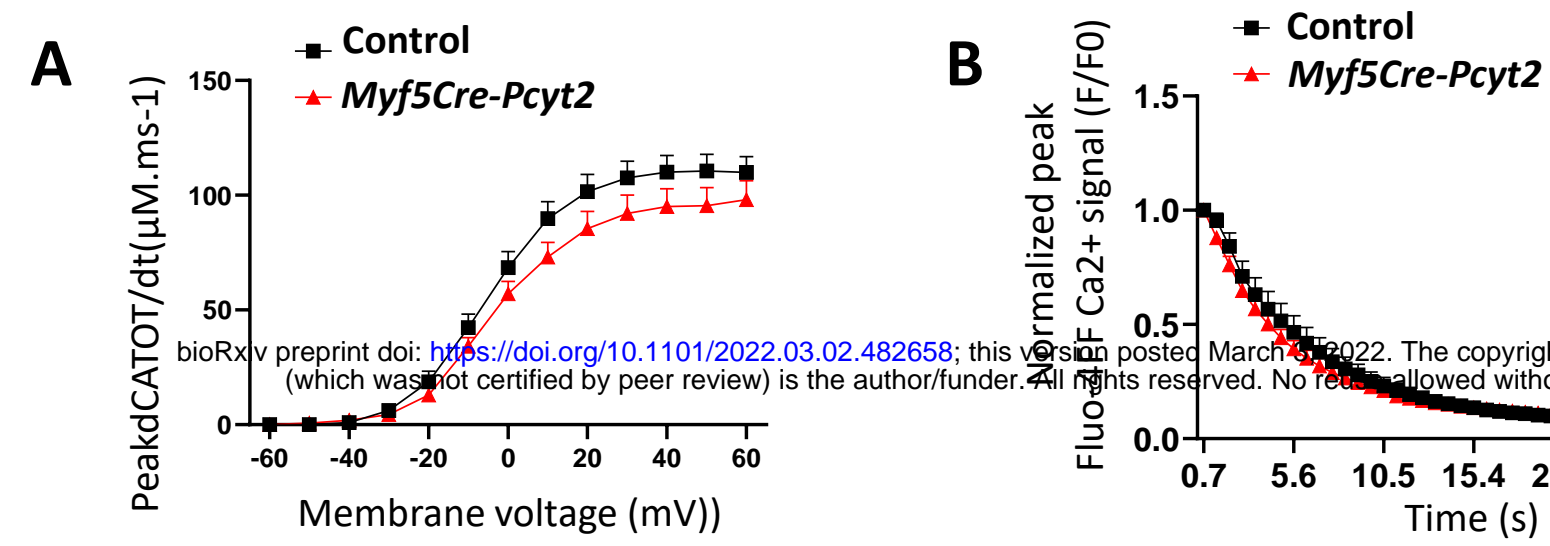

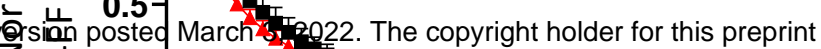

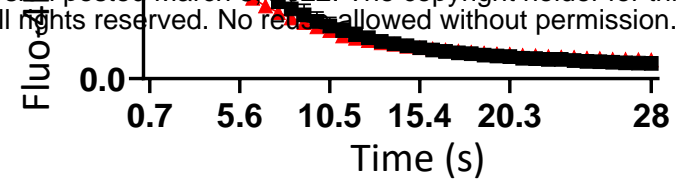

C

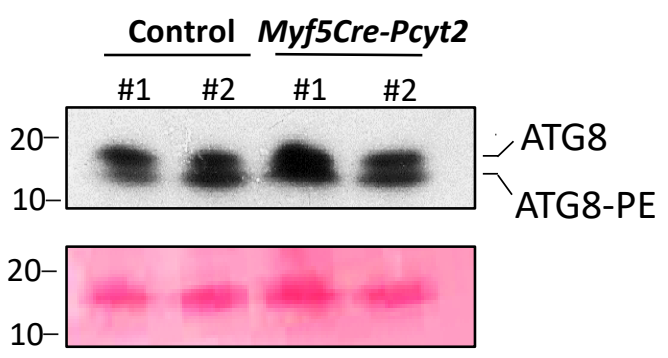

D Control Myf5Cre-Pcyt2 E

- Control

$\triangle$ Myf5Cre-Pcyt2
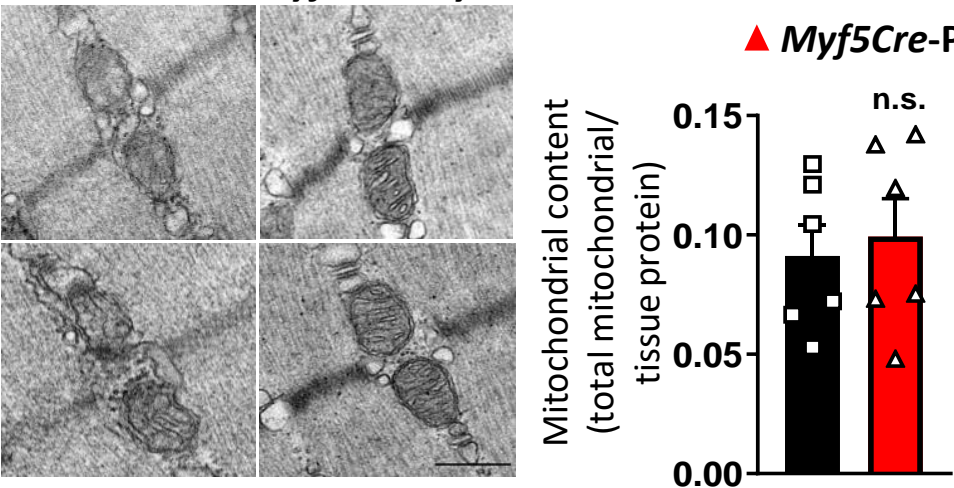

$\mathbf{F}$

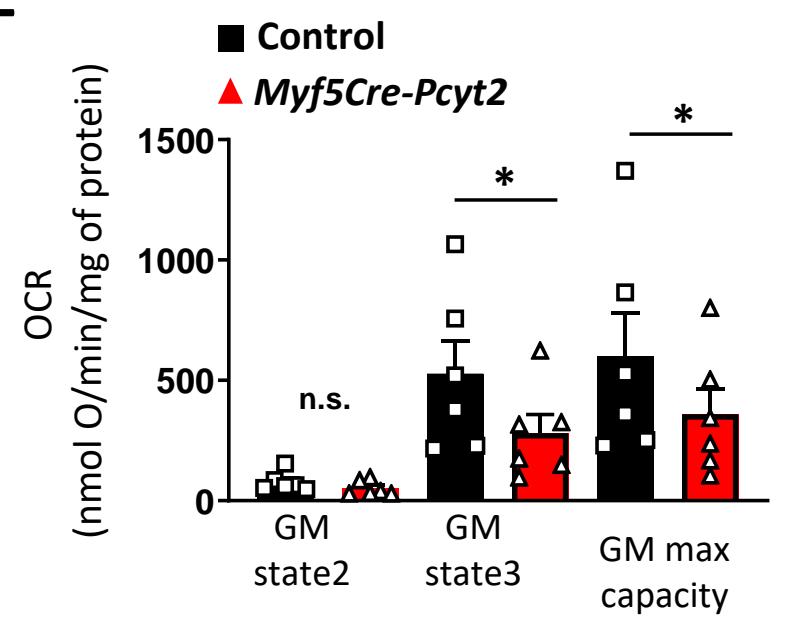

H

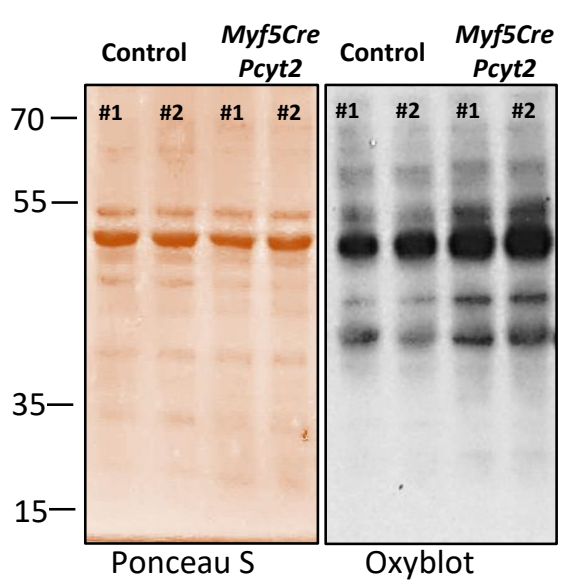

- Control

$\triangle$ Myf5Cre-Pcyt2

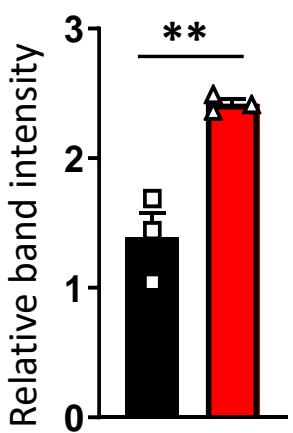

$\mathbf{G}$
J

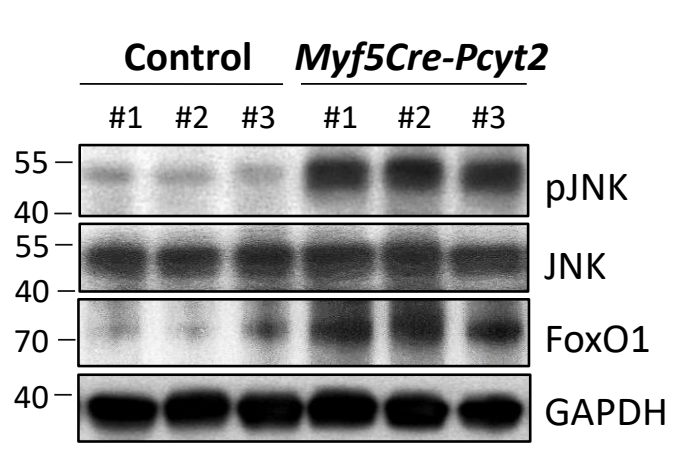

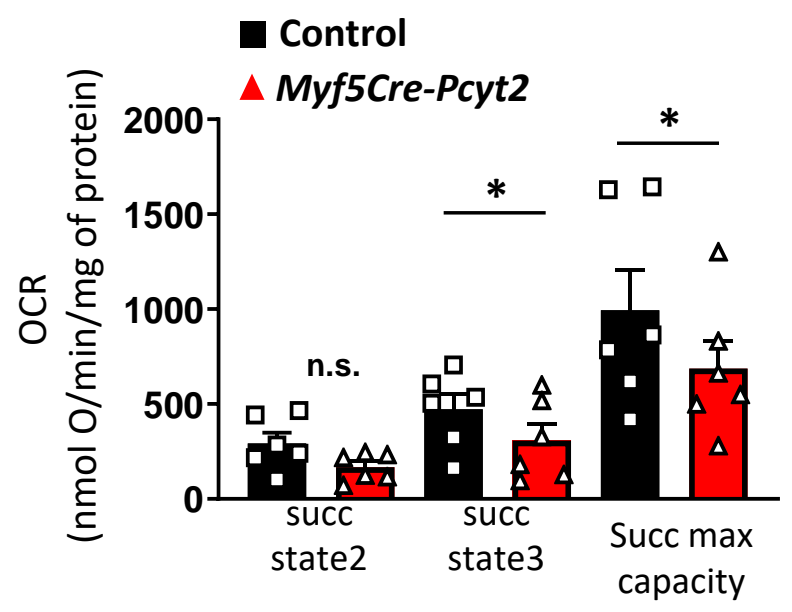

I
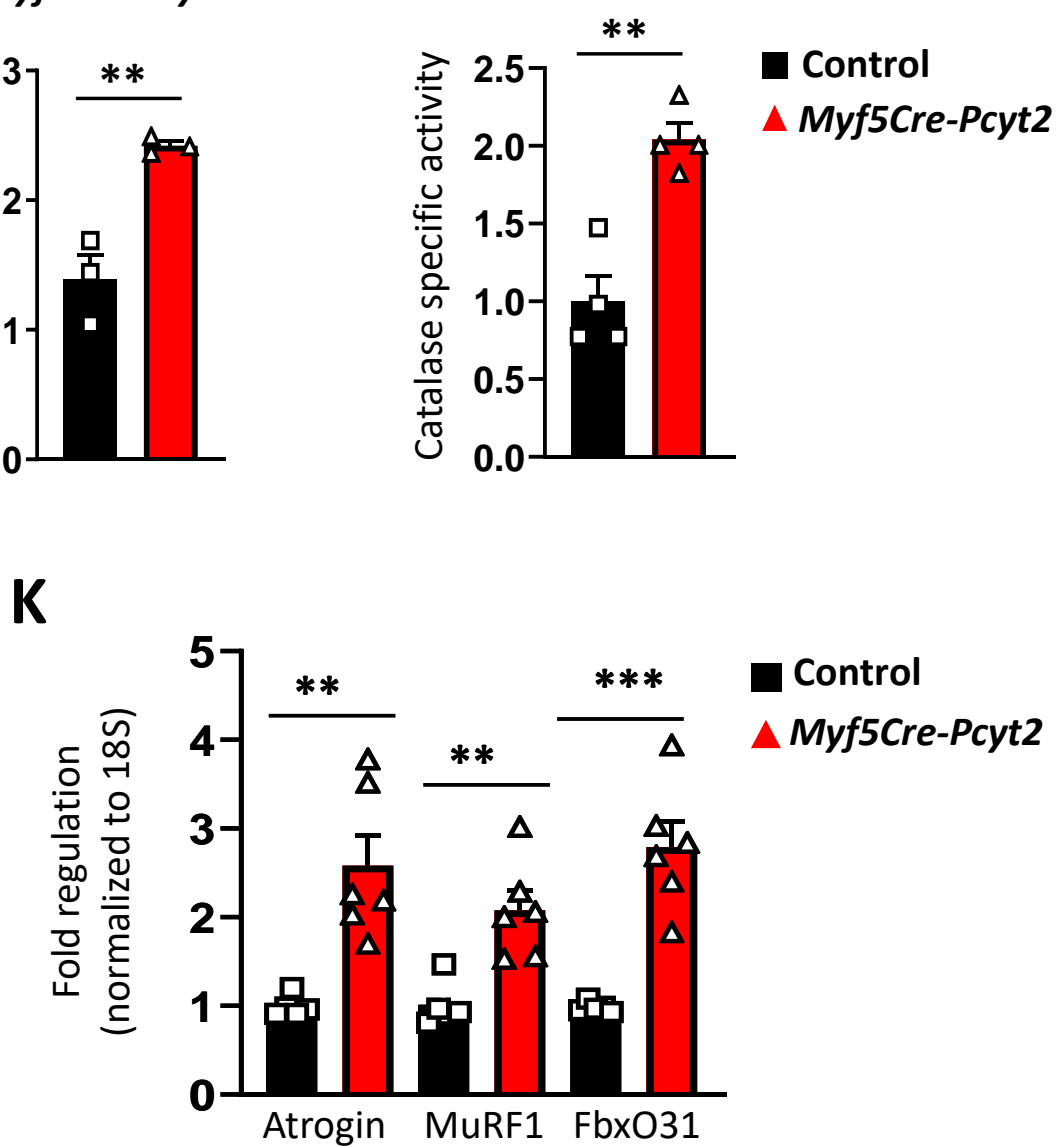


\section{Extended Data Figure 9}

A

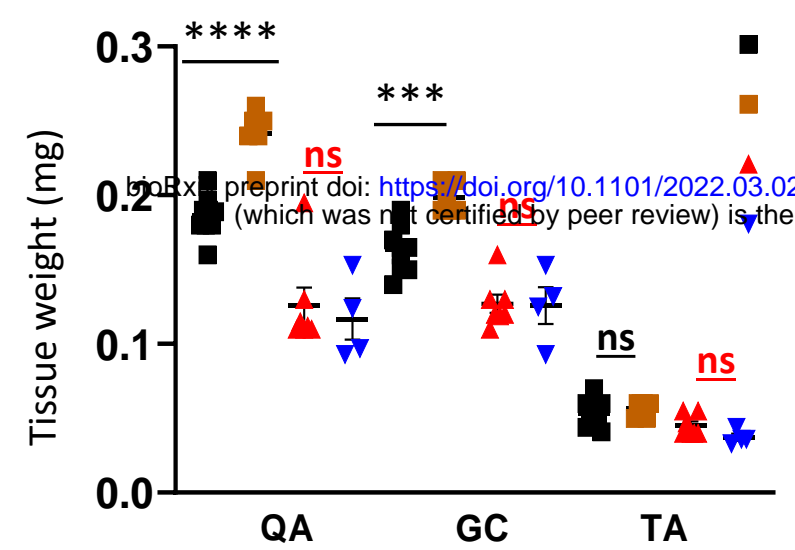

B

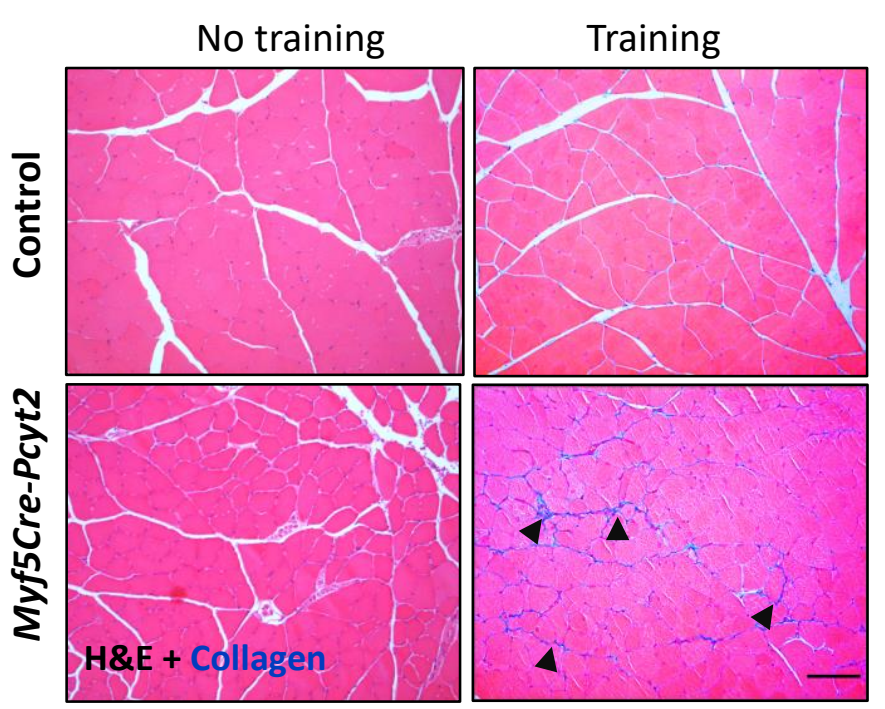

C

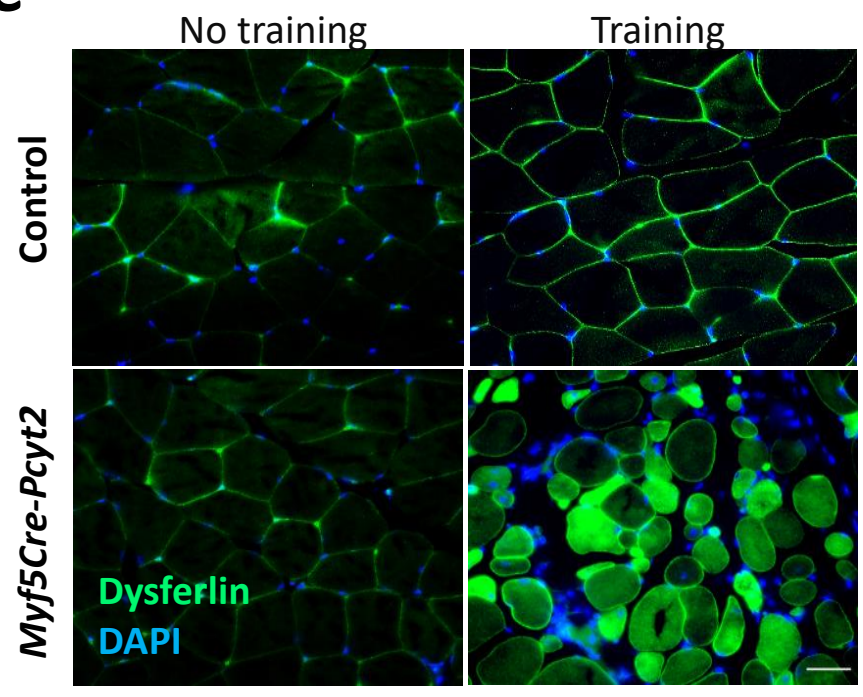

D

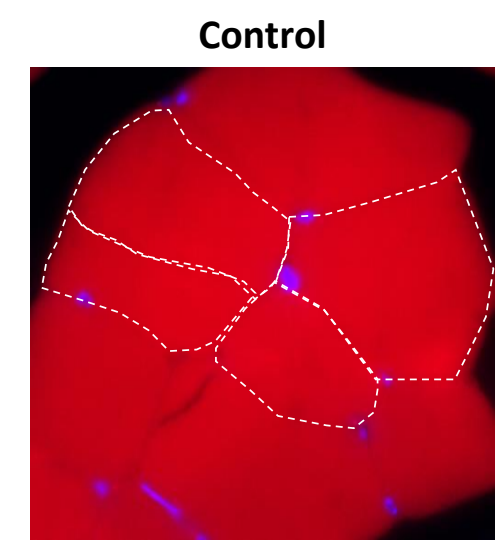

Myf5Cre-Pcyt2 KO
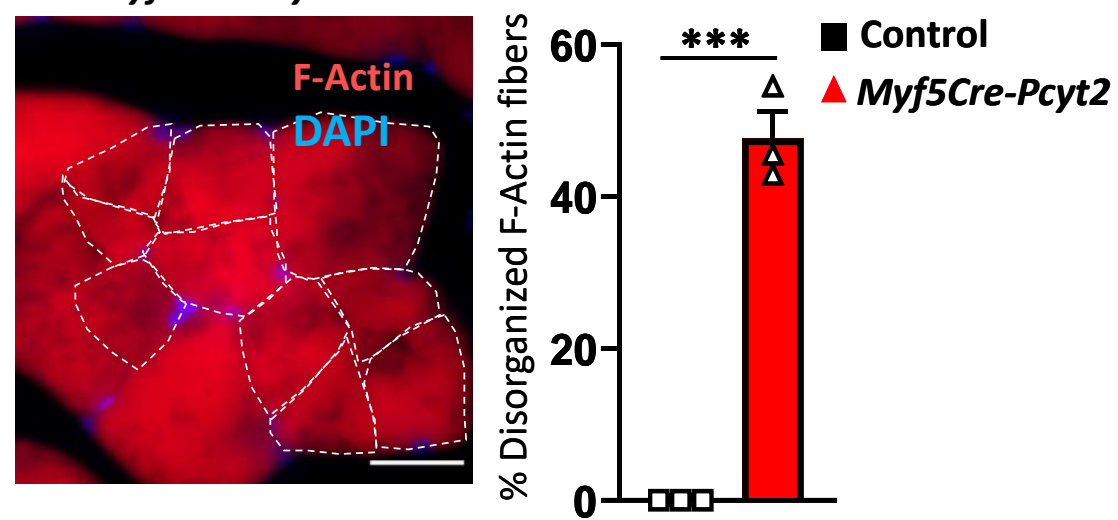

E

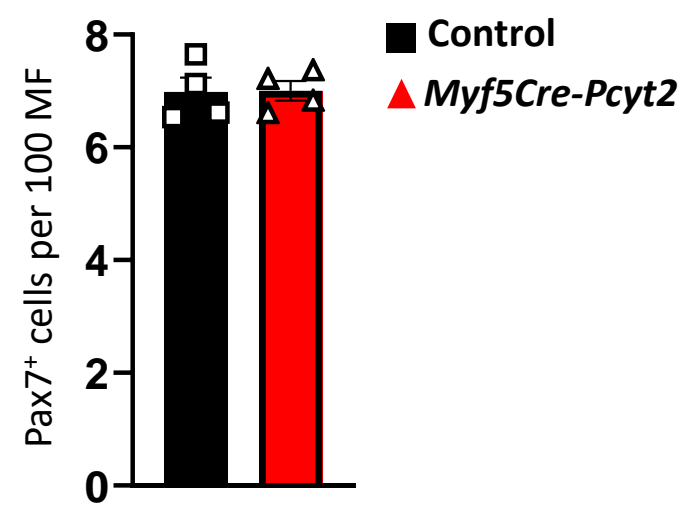

Control

yf5Cre-Pcyt2 2 ; 2 ;

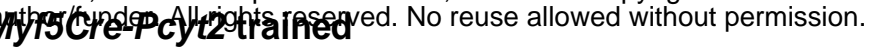




\section{Extended Data Figure 10}

A

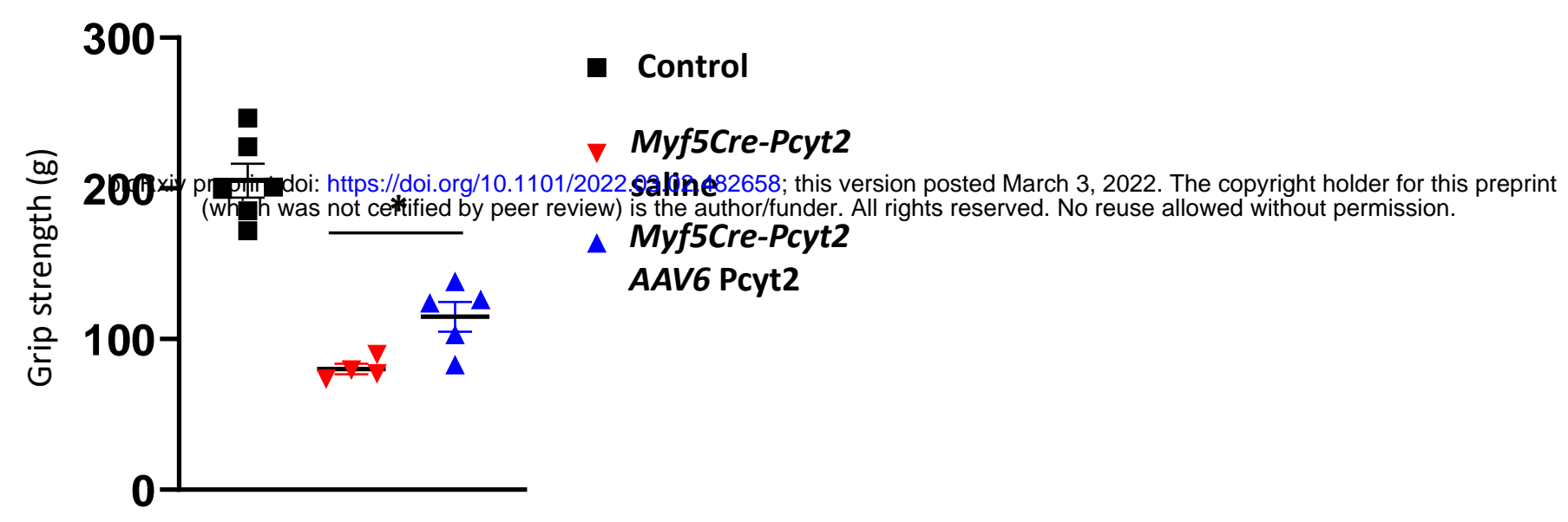

B

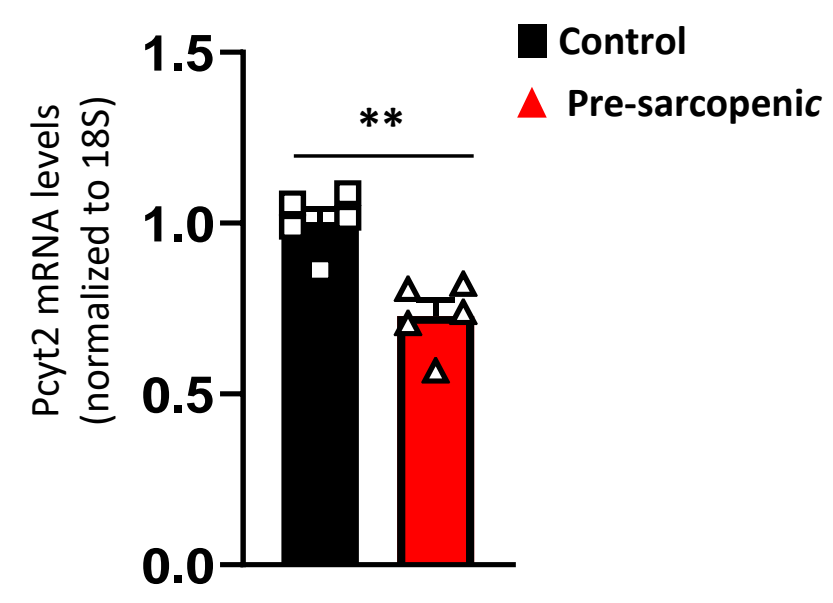

C
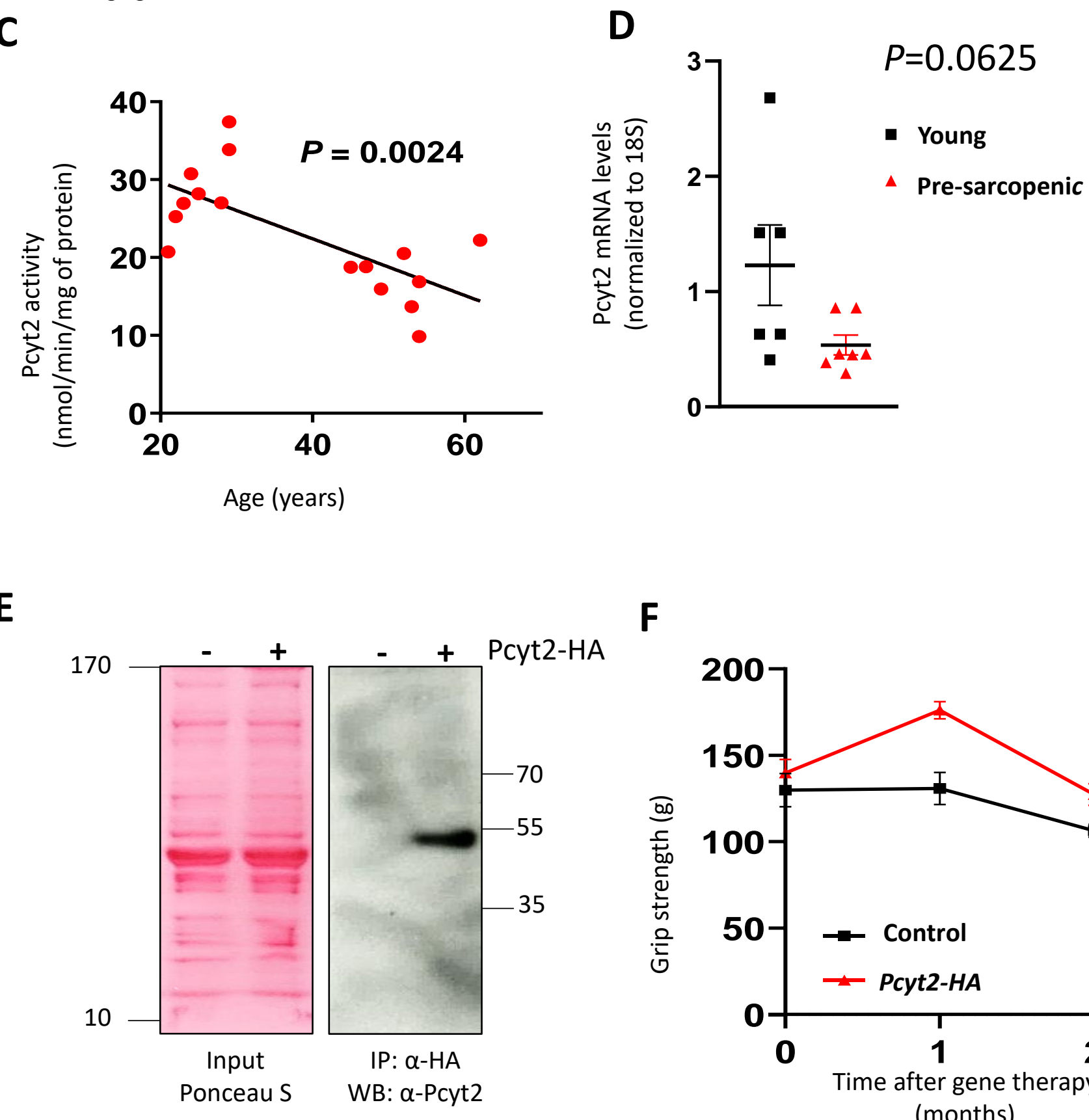

F

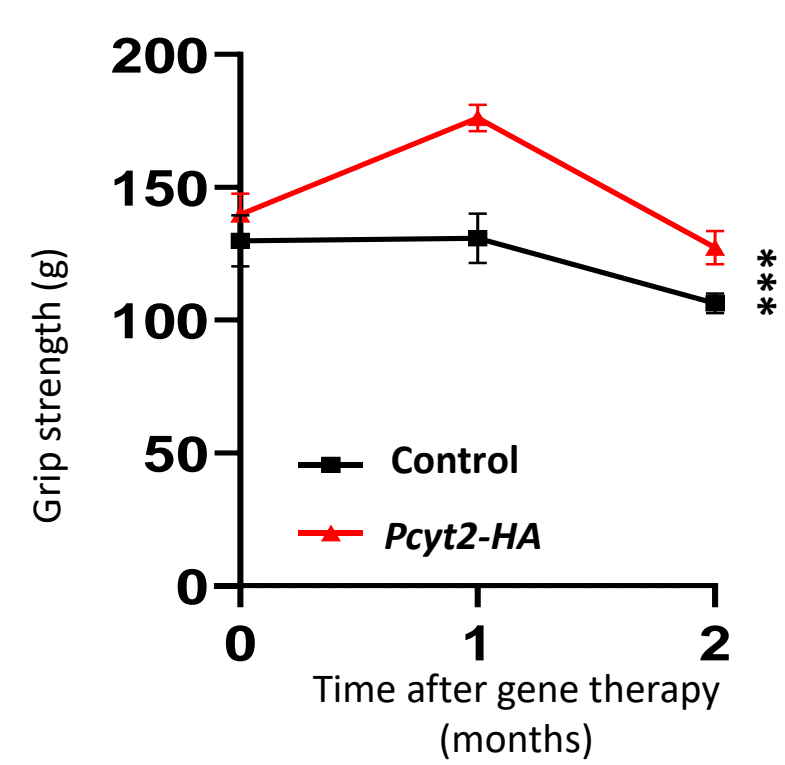

